\title{
Dynamic Optical Investigations of Hypervelocity Impact Damage
}

\author{
Thesis by \\ Leslie Elise Lamberson \\ In Partial Fulfillment of the Requirements \\ for the Degree of \\ Doctor of Philosophy
}

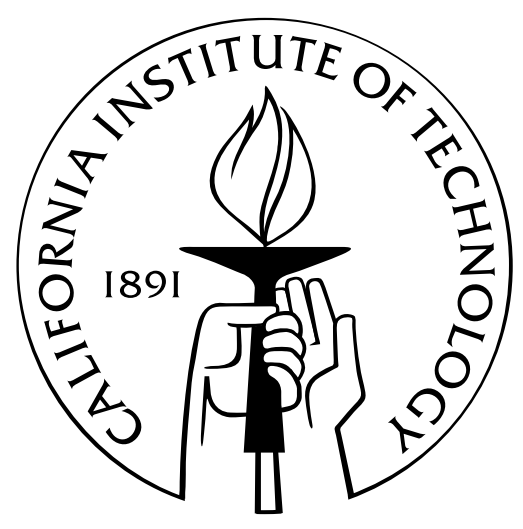

California Institute of Technology

Pasadena, California

2010

(Defended May 21, 2010) 
(c) 2010

Leslie Elise Lamberson

All Rights Reserved 
To my dad. 


\section{Acknowledgements}

Everything in the universe has rhythm. Everything dances.

-Maya Angelou

I would like to first acknowledge my advisor, Ares Rosakis, for his support and gracious role in developing my academic career. I also gratefully acknowledge the support of my thesis committee, Chiara Daraio, Julia Greer, Michael Ortiz, and Guruswami Ravichandran. I would like to particularly thank Dr. Ravi for his generous guidance thoroughout my doctoral studies, and Dr. Ortiz for constant encouragement of my research endeavors. I would also like to thank the professors who made my journey to Caltech possible: Arthur Hoadley from Western Michigan University, Anne Marie Sastry and Tony Waas from my undergraduate studies at the University of Michigan, and George Kardomateas and Erian Armanios from my masters work at the Georgia Institute of Technology.

The largest and most heartfelt thank you goes to my mom and dad who, in my humble opinion, are the best two people on the planet. To my brother Jonathan, Beverly, Max, Becky and Amit, Aunt Jo Anne and Uncle Andy, Aunt Kay and Uncle Frank, Debbie and my nieces Phoebe and Annabel, and Nana and Papa in spirit, thank you for being such a supportive family. A particular thank you goes to my cousin Chris, whose support and company in the doctoral undertaking is immensely appreciated.

A huge thank you goes to Veronica Eliasson for her mentoring and friendship. I would have nothing to write about if it wasn't for her. To the Caltech group who were there for me in various ways: Angie Capece, an incredible officemate and wonderful confidant, Roseanna Zia, an amazing mentor and gracious friend, Catherine Beni, one of the smartest and most patient (particularly when explaining math to yours truly) people I know, to Andre Yavrouian and Tim O'Donnell for always making my visits to JPL pleasant ones, and to Dr. Jay for helping me get my research started when things were at a frustrating standstill. A special acknowledgement to my friend Jason Johnson, one of the most generous souls I have met during my time here. I gratefully acknowledge friends not in Pasadena, 
but who still support me from afar: Big Mere, Lil' Mere, Adele, Danielle, Austin, and Eric. To my adopted godmom, Amy Terry, thanks for always believing in me. To my Texas buddy Terry, over the years your friendship and support of my career has meant so much. I would also like to thank the Aeronautics machine shop crew, Joe, Brad, and Ali, with special thanks to Brad for often working extra just to make things easier on me. I would like to thank Joe Areeda for literally showing me how to fly, and for his support in my education even if it meant less plane time. In industry, I would like to thank my former Lockheed teams in Fort Worth working with Mike Love and Scott Zink, in Marietta under Skip Ellsworth, the stress engineers presently and some formerly under Brad Hanson in Palmdale, and the tremendous group in hypersonics at NASA Ames. I learned so much from you all, and appreciated each experience. Additionally, a special thank you to Dimity, Lydia, and Donna for the support that went above and beyond merely administrative. I would also like to give huge thanks to Peet's Coffee crew store 301. You all made me exceptional beverages, but more importantly, you all have been exceptionally kind. Special thanks to Frank, the most delightful companion of the feline nature.

To Cynthia Young and Lawrence Blake at Pasadena Dance Theatre, I cannot thank you enough for helping me develop my technique and allowing me to perform with the company. I was given the opportunity to dance roles I had only ever dreamed of, and it has truly been an honor. To the best group of dancers and friends I worked with at PDT - all the girls, and specifically Iris, Gustavo, Preston, Alanna, Ashleigh, Marcos, Mandy, Chloe, Jesse, Anna, Brian, Jin and Benny, thank you for all the good times, even during long rehearsals, and especially for all the memorable performances. To Ms. Helga deKansky and the fantastically talented women in adult ballet, particularly Kelly, Barbara, Holly, Valerie, and Janis, I couldn't have asked for a better cheering section and often 'mom-away-from-mom' care. To Christian Perry and the brilliant team at Rose City Ballroom, thank you for giving me the opportunity to pursue a new form of dance, and for the unwaivering confidence in my efforts inside and outside the ballroom world. I now hope to cha-cha my way through life.

I also gratefully acknowledge all past and present research support including the Caltech Center for Predictive Science Academic Alliance Program with support from the Department of Energy Award Number DE-FC52-08NA28613 through the National Nuclear Security Administration, the Zonta International Amelia Earhart Fellowship, the National Science Foundation Graduate Research Fellowship, and the NASA Aeronautics Scholarship Program through the American Society of Engineering Education.

Last, but certainly not least, a most sincere thank you to Philipp Boettcher who is, to me, the best part of Caltech, and who probably knows more about hypervelocity impact research than he ever wanted to. Ein herzliches Dankeschön! 


\section{Abstract}

One of the prominent threats in the endeavor to develop next-generation space assets is the risk of space debris impact in earth's orbit and micrometeoroid impact damage in near-earth orbit and deep space. To date, there is no study available which concentrates on the analysis of dynamic crack growth from hypervelocity impacts on such structures, resulting in their eventual catastrophic degradation. Experiments conducted using a unique two-stage light-gas gun facility have examined the in situ dynamic fracture of brittle polymers subjected to this high-energy-density event. Optical techniques of caustics and photoelasticity, combined with high-speed photography up to 100 million frames per second, analyze crack growth behavior of Mylar and Homalite 100 thin plates after impact by a $1.8 \mathrm{~mm}$ diameter nylon 6-6 right cylindrical slug at velocities ranging from 3 to $7 \mathrm{~km} / \mathrm{s}$ (7000-15500 mph). Crack speeds in both polymers averaged between 0.2 and $0.47 c_{R}$, the Rayleigh wave speed (450-1000 mph). Shadow spots and surrounding caustics reveal time histories of the dynamic stress intensity factor, as well as the energy release rate ahead of the mode-I, or opening, crack tips. Results indicate that even under extreme impact conditions of out-of-plane loading, highly localized heating, and energetic impact phenomena involving plasma formation and ejecta, the dynamic fracture process occurs during a deformation regime dominated by in-plane loading. These findings imply that the reliability of impacted, thin-walled, plate and shell space structures, idealized by the experimental configuration investigated, can be predicted by the well-defined principles of classical dynamic fracture mechanics. 


\section{Contents}

Acknowledgements $\quad$ iv

Abstract $\quad$ vi

1 Introduction 1

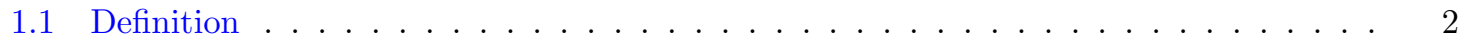

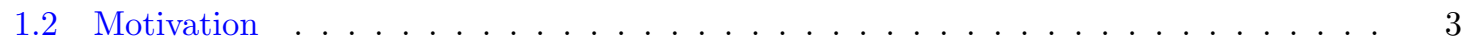

1.3 Impact Physics . . . . . . . . . . . . . . . . . . . . . 8

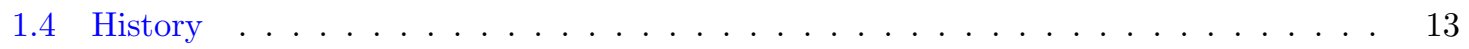

1.5 Test Facility . . . . . . . . . . . . . . . . . . . . . . . . . 14

1.6 Launch Mechanics . . . . . . . . . . . . . . . . . . . . . . . 18

1.7 Facility Capability . . . . . . . . . . . . . . . . . . . . . 20

1.8 Facility Efficiency . . . . . . . . . . . . . . . . . . . . . . . . . 24

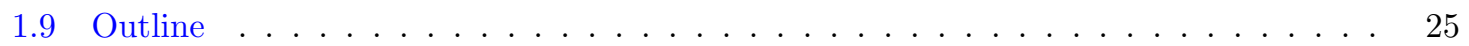

2 Hypervelocimetry 27

2.1 Detection and Error $\ldots \ldots \ldots \ldots \ldots \ldots \ldots \ldots$

2.2 Method 1: Mylar Flash Method . . . . . . . . . . . . . . . . . . 33

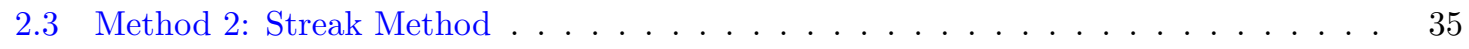

2.4 Velocimetry Comparison . . . . . . . . . . . . . . . . . . . 36

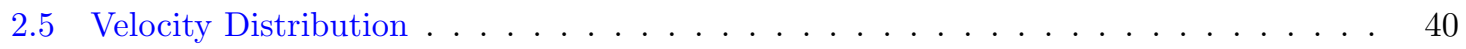




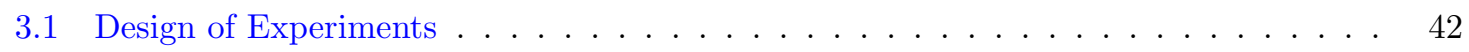

3.2 Analysis of Variance Model f . . . . . . . . . . . . . . . . . . . . . . . 43

3.3 Full-Factorial Results . . . . . . . . . . . . . . . . . . . . . . . . . . . . . . 48

3.4 Validation of ANOVA Model . . . . . . . . . . . . . . . . . . . . . 50

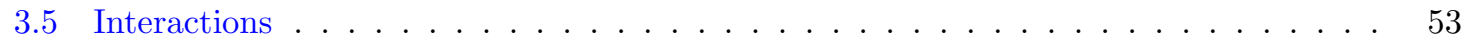

3.6 First-Order Hypervelocimetry Model . . . . . . . . . . . . . . . . . . . . . . 54

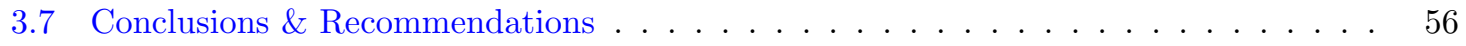

$\begin{array}{lll}4 & \text { Terminal Ballistics } & 57\end{array}$

4.1 Ballistic Limit Equations $\ldots \ldots \ldots \ldots \ldots \ldots$

4.2 Modified Charters Equation . . . . . . . . . . . . . . . . . 60

4.3 Case Study . . . . . . . . . . . . . . . . . . . . 64

$\begin{array}{lll}5 & \text { Dynamic Optical Investigations } & 67\end{array}$

5.1 Dynamic Fracture Mechanics . . . . . . . . . . . . . . . . . . . . . . 68

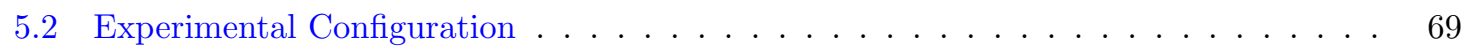

5.3 Optical Methods . . . . . . . . . . . . . . . . . . . . . . 71

5.4 Results \& Conclusions . . . . . . . . . . . . . . . . . . . . . . . . 76

$\begin{array}{lr}\text { A Additional Statistical Analysis } & 88\end{array}$

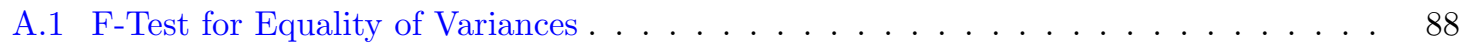

A.2 Anderson-Darling Test for Normality . . . . . . . . . . . . . . . . . . . . 89

A.3 Levene Test for Equality of Variances _ . . . . . . . . . . . . . . . . . . 89

A.4 Velocity Distributions . . . . . . . . . . . . . . . . . . . . . 90

B Additional Optical Investigation Details \& Extensions $\quad 95$

B.1 Optical Distortion Removal Code . . . . . . . . . . . . . . . . . . . . 95 
ix

B.2 Extensions of Presented Dynamic Optical Investigations . . . . . . . . . . . . . 96

Bibliography 


\section{List of Tables}

$1.1 \quad$ Potential adverse effects of hypervelocity impacts on space assets . . . . . . . . . . 6

1.2 Hypervelocity impact penetration data from ten space science satellites . . . . . . . 7

1.3 Table of spacecraft damaged by or suffering complete failure from space debris and

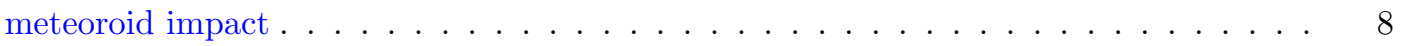

1.4 Hypervelocity impact conditions examined in various thin plate test databases . . . . 14

3.1 Hypervelocity observations from DOE tests varying launch package, hydrogen pressure, and vacuum levels on two-stage light-gas gun . . . . . . . . . . . . . . . 45

3.2 The analysis of variance table for three-factor fixed effects two-stage light-gas gun model 47

3.3 The analysis of variance table for three-factor fixed effects gas-gun experiment _ . . . 49

4.1 Table of thermal protection system material hypervelocity impact damage penetration response from NASA Ames case study . . . . . . . . . . . . . . . . . . 65

5.1 Elastic and optical properties of Homalite 100 and Mylar . . . . . . . . . . . . . 74

5.2 Results from optical investigations of hypervelocity impact dynamic fracture of brittle

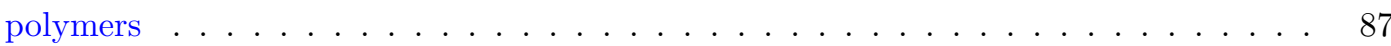




\section{List of Figures}

1.0.1 Cumulative end-of-year launches reaching earth's orbit or beyond and cumulative endof-year satellite objects in orbit from 1957 to 1994 . . . . . . . . . . . . . . . . 2

1.2.1 Cataloged near earth orbiting population from December 8, 1989 . . . . . . . . 3

1.2.2 LEO and GEO orbital population image, from NASA's Orbital Debris Program Office in 2010 . . . . . . . . . . . . . . . . . . . . . . . . 5

1.2.3 Space shuttle Endeavour impact tile damage on Orbital Maneuvering System (OMS), and window hypervelocity impact damage from space shuttle Challenger . . . . . . .

1.3.1 Simplified model of hypervelocity impact of right cylinder on semi-inifinite plate at initial velocity, $\mathrm{v}_{0}$, and $0^{\circ}$ obliquity . . . . . . . . . . . . . . . . 10

1.3.2 Hypervelocity impact process on a pressure-volume plot . . . . . . . . . . . . . 12

1.3.3 One-dimensional representation of the shocking and rarefaction wave process immediately after impact with coordinates fixed on the plate . . . . . . . . . . .

1.3.4 Time-distance ( $\mathrm{x}$ - $\mathrm{t}$ ) diagram illustrating hypervelocity impact shock and rarefaction

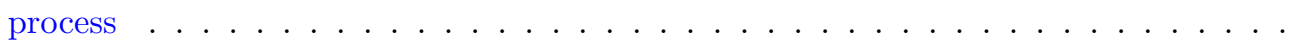

1.5.1 Two-stage light-gas gun facility viewed down the shot line from trigger pull to target

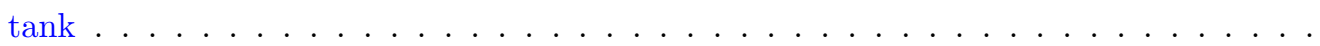

1.5.2 Working envelope of two-stage light-gas gun impactor velocity and mass for certain

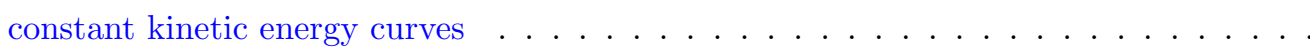

1.5.3 Working envelope of two-stage light-gas gun kinetic energy density for usable span of impactor mass, assuming a $1.8 \mathrm{~mm}$ diameter sphere volume . . . . . . . . . . . .

1.6.1 Schematic of the two-stage light-gas gun viewed directly from the side . . . . . . .

1.6.2 Photograph illustrating AR separation between stage 1 and stage 2 of the two-stage light-gas gun used for experiments . . . . . . . . . . . . . . . . . . 
1.6.3 Example spherical and cylindrical aluminum, steel, polycarbonate, and nylon impactors, and sectioned impactors on a penny for scaling perspective . . . . . . . . . 20

1.7.1 (A) Tantalum sphere, $1.8 \mathrm{~mm}$ diameter, embedded into 304 stainless steel plate $\quad$. . 21

1.7.2 (A) Tantalum right cylindrical slug, $1.8 \mathrm{~mm}$ and length, before impact. (B) Tantalum right cylindrical slug after impact with Homalite 100 plate . . . . . . . . . . .

1.7.3 Bright-field TEM image of (B) from Figure 1.7.2, after being impacted against Homalite

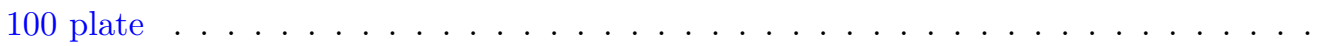

1.7.4 Nylon right cylindrical slug, $1.8 \mathrm{~mm}$ in length and diameter, having impacted Aluminum 6061-T6 plate $150 \mathrm{~mm} \times 150 \mathrm{~mm} \times 25 \mathrm{~mm}$ at $6 \mathrm{~km} / \mathrm{s} \ldots \ldots$. . . . . . .

1.7.5 Plates of 1018 steel, $150 \mathrm{~mm}$ diameter and $10 \mathrm{~mm}$ thick, impacted at (A) $0^{\circ}$ obliquity

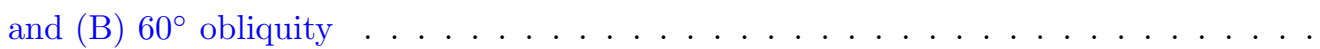

2.1.1 Velocimetry configuration for two-stage light-gas gun . . . . . . . . . . .

2.1.2 Trigger mechanism used for high-speed velocimetry in the gas-gun facility. $Q_{1}$ sends a $5 \mathrm{~V}$ DC output upon excitation at a variable threshold region, $\mathrm{R}_{1} \ldots$. . . . . . .

2.1.3 Muzzle blast structure on the downrange end of the launch tube which can affect

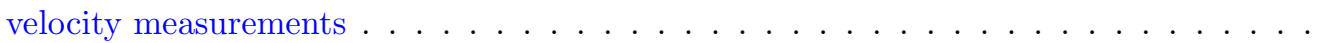

2.1.4 Photodiode trigger velocity system output from a hypervelocity impact test illustrating the difference in diode response time . . . . . . . . . . . . . . . . . . .

2.1.5 Example of forward ejecta captured by high-speed photography from a hypervelocity impact event viewed from the side . . . . . . . . . . . . . . . . . .

2.1.6 Shadowgraph of ejecta (A) $30 \mu$ s after impact and (B) $35 \mu$ s after impact with nylon cylindrical slug, $1.8 \mathrm{~mm}$ in diameter and length, on Mylar plate . . . . . . . . . .

2.1.7 Example of forward and rearward ejecta, spectral flash, and plasma glow captured by high-speed photography viewed from the side of a hypervelocity impact event . . . . .

2.2.1 Mylar flash velocimetry method showing a 440C stainless steel sphere impactor, $1.8 \mathrm{~mm}$ in diameter, colliding with a 304 stainless steel plate . . . . . . . . . . . . .

2.3.1 Streak velocimetry method showing plasma sheath around nylon cylindrical impactor, $1.8 \mathrm{~mm}$ in diameter and length, at a series of instances in time . . . . . . . . . . 
xiii

2.4.1 Graph of hypervelocity impact tests using nylon cylindrical slugs, $1.8 \mathrm{~mm}$ length and diameter, with velocity readings for both the Mylar flash and streak methods . . . . .

2.4.2 Graph of hypervelocity impact tests using tantalum sphere, $1.8 \mathrm{~mm}$ diameter, with velocity readings for both the Mylar flash and streak methods . . . . . . . . . . .

2.4.3 Graph of the difference between the Mylar flash method and the streak method of measuring hypervelocity . . . . . . . . . . . . . . . . . . .

2.4.4 High-speed photography shadowgraph of a bow shock ahead of a moving nylon cylindrical impactor, $1.8 \mathrm{~mm}$ in diameter, traveling at $4.23 \mathrm{~km} / \mathrm{s}$. . . . . . . . . . . .

2.5.1 Normal distribution probability plot of velocity for nylon cylindrical projectiles, $1.8 \mathrm{~mm}$ in diameter and length, with a $95 \%$ confidence interval . . . . . . . . . . . . . .

2.5.2 Normal distribution probability plot of velocity for tantalum sphere projectiles, $1.8 \mathrm{~mm}$ in diameter, with a $95 \%$ confidence interval . . . . . . . . . . . . . .

3.2.1 Sum of squares partitioning for three-way analysis of variance model . . . . . . . .

3.4.1 Normal probability plot of residuals in two-stage light-gas gun ANOVA DOE . . . . .

3.4.2 Residuals versus the order of the recorded observations in two-stage light-gas gun

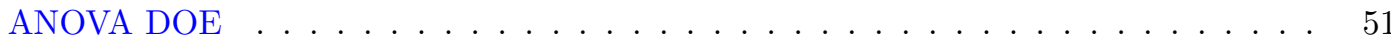

3.4.3 F-test and Levene's Test to examine ANOVA model equivalence of variance . . . . . . 52

3.4.4 Residuals versus fitted values . . . . . . . . . . . . . . . . . . . . 53

3.5.1 Factor interaction plot of gas-gun ANOVA DOE . . . . . . . . . . . . . . 54

3.6.1 Fitted line plot of DOE data, mass of launch package versus resulting velocity . . . . 55

3.6.2 Regression analysis, fitted curve to extended DOE data, mass of launch package versus

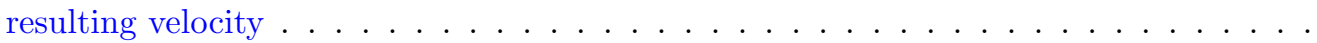

4.2.1 Plot illustrating failure from strong compressive wave with resolved shear stress for idealized brittle material . . . . . . . . . . . . . . . . . . . .

4.2.2 440C steel sphere perforation results from impact with 304 stainless steel plates at

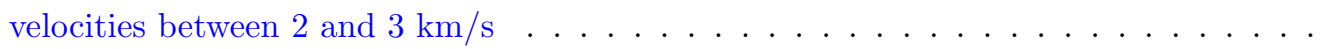

4.2.3 Tantalum sphere perforation results from impact with 304 stainless steel plates at

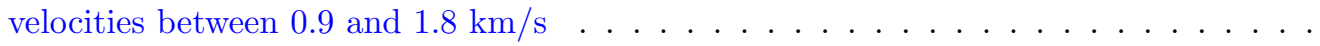


4.2.4 Charters, modified Charters, and experimental results comparison from tantalum sphere impactors, $1.8 \mathrm{~mm}$ in diameter, hole perforation size on 304 stainless steel plates . . .

4.3.1 NASA Ames case study results of hypervelocity impacts on Fiberform and LI-2200 . .

5.0.1 Hubble Space Telescope solar cell hypervelocity impact damage versus laboratory generated hypervelocity impact damage of Homalite $100 \ldots \ldots$. . . . . . . . . . . . . 67

5.2.1 Dynamic optical experimental configuration using two-stage light-gas gun facility . . . 70

5.2.2 Photographs of the dynamic optical analysis using the two-stage light-gas gun showing (A) the optical path and (B) the target plate with notch in load frame . . . . . . . . 71

5.3.1 Schematic of information obtained by the methods of dynamic photoelasticity and

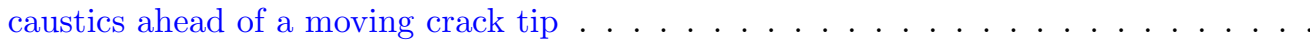

5.3.2 (A) The actual grid as seen by the high-speed camera of the $100 \mathrm{~mm}$ expanded laser beam, taken to remove optical distortions by using a (B) virtual grid . . . . . . . . . 72

5.3 .3 The method of shadow spots from caustics in transmission $\ldots \ldots \ldots \ldots$

5.3.4 Schematic of circular polariscope configuration for photoelasticity . . . . . . . . 75

5.4.1 Homalite 100 resulting crack path appearance. Nearly all cases exhibited an oscillating crack path, except in the highest velocity crack speed cases . . . . . . . . . . . 77

5.4 .2 Mylar resulting flat crack path appearance . . . . . . . . . . . . . . . 77

5.4.3 Microscopy images of polymer hypervelocity impact sites of Homalite 100 and Mylar . 78

5.4.4 High-speed photography is used to capture the isochromatic fringe pattern, caustics and shadow spots generated during crack growth resulting from impact on Mylar . . .

5.4.5 High-speed photography is used to capture the isochromatic fringe pattern, caustics and shadow spots generated during crack growth resulting from impact on Homalite 10080

5.4.6 High-speed photography is used to capture the isochromatic fringe pattern, caustics and shadow spots generated during crack growth resulting from impact on Mylar . . .

5.4.7 Dynamic stress intensity factor (mode-I) at the crack tip as a function of crack speeds resulting from hypervelocity impact induced fracture on Mylar thin plates . . . . . . .

5.4.8 Averaged dynamic stress intensity factor (mode-I) at the crack tip as a function of crack speeds resulting from hypervelocity impact induced fracture on Homalite 100 thin plates 83 
5.4.9 Dynamic stress intensity factor versus crack tip speeds in Homalite 100 for various geometries of classical mode-I configurations . . . . . . . . . . . . . .

5.4.10 Averaged dynamic energy release rates at the crack tip as a function of crack speeds resulting from hypervelocity impact induced fracture on Mylar thin plates . . . . . . .

5.4.11 Averaged dynamic energy release rates at the crack tip as a function of crack speeds resulting from hypervelocity impact induced fracture on Homalite 100 thin plates . . .

A.4.1 Additional distributions fit to nylon 6-6 cylindrical slug, $1.8 \mathrm{~mm}$ length and diameter, hypervelocimetry data . . . . . . . . . . . . . . . . . . .

A.4.2 Additional distributions fit to nylon 6-6 cylindrical slug, $1.8 \mathrm{~mm}$ length and diameter, hypervelocimetry data . . . . . . . . . . . . . . . . . . . .

A.4.3 Additional distributions fit to nylon 6-6 cylindrical slug, $1.8 \mathrm{~mm}$ length and diameter, hypervelocimetry data . . . . . . . . . . . . . . . . . . . .

A.4.4 Additional distributions fit to tantalum spheres, $1.8 \mathrm{~mm}$ diameter, hypervelocimetry

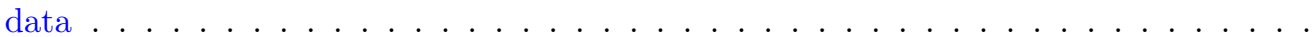

A.4.5 Additional distributions fit to tantalum spheres, $1.8 \mathrm{~mm}$ diameter, hypervelocimetry

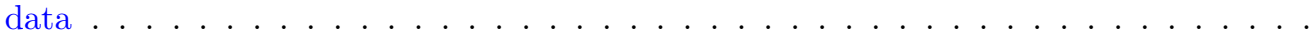

A.4.6 Additional distributions fit to tantalum spheres, $1.8 \mathrm{~mm}$ diameter, hypervelocimetry

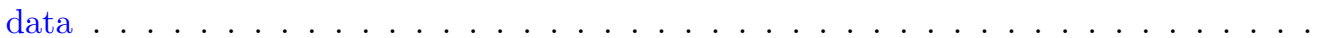

A.4.7 Additional distributions fit to 440C steel spheres, $1.8 \mathrm{~mm}$ diameter, hypervelocimetry

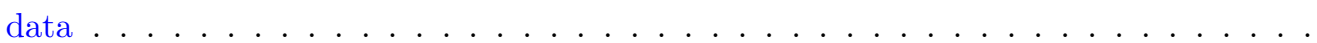

A.4.8 Additional distributions fit to 440C steel spheres, $1.8 \mathrm{~mm}$ diameter, hypervelocimetry

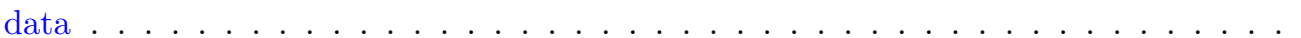

A.4.9 Additional distributions fit to 440C steel spheres, $1.8 \mathrm{~mm}$ diameter, hypervelocimetry

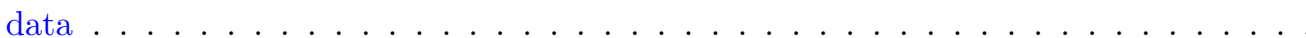

B.2.1 Multiple hypervelocity impact strike cumulative damage shown by dynamic photoelas-

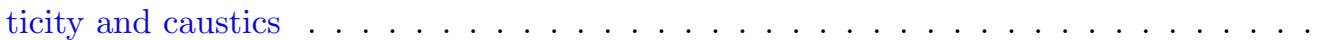

B.2.2 Post-mortem Coherent Gradient Sensing, shearing interferometry technique, slope and topology results from hypervelocity impact on a steel plate . . . . . . . . . . . 
xvi

B.2.3 In situ Coherent Gradient Sensing, shearing interferometry technique, results from hypervelocity impact on a brittle polymer plate . . . . . . . . . . . . . .

B.2.4 Homalite 100, $10 \mathrm{~mm}$ thick, weakly interfaced with Homalite 100, $10 \mathrm{~mm}$ thick, and impacted on the lower portion by a nylon cylindrical slug at $6.3 \mathrm{~km} / \mathrm{s}$. . . . . . . . . 100

B.2.5 Optical diagnostics during impact of a Homalite 100 interface by a nylon cylindrical slug at $6.3 \mathrm{~km} / \mathrm{s} \ldots \ldots \ldots \ldots \ldots 1 . \ldots \ldots \ldots$ 


\section{Chapter 1}

\section{Introduction}

With the future of space exploration and space science at the mercy of a nation's political climate, economic situation, and human interest, the development of space assets that are increasingly robust, mission-capable, and cost-effective is imperative. In recent years, one of the most prominent threats in this endeavor is the risk of space debris damage within earth's orbit, as well as micrometeoroid impacts in near earth and deep space missions. Meteoroids are defined generally as rock, mineral or ice-like material particles found in solar orbit, and space debris is considered non-functional manmade material residing within low earth (LEO), semisynchronous (SEO), and geosynchronous orbit (GEO). The most common space debris comprises of billions of tiny aluminum particles from solid rocket fuel waste. Additionally, inactive payloads, discarded rocket stages, and coolant released by nuclear powered satellites from early space-history can be categorized as space debris [41]. The latter type of debris hardly remains in original form, often shedding lens, boosters, nuts, bolts, paint-chips, foil particles, as well as continually colliding with other existing space debris, creating additional smaller pieces. Figure 1.0.1 illustrates the extent the near region of space has been exploited since the launch of Sputnik in 1957. While human activities in space have increased over the past four decades, so does civilization's dependence on orbiting space assets; yet at the same time these assets are becoming increasingly vulnerable to the growing population of orbital debris. For example, the International Space Station includes over one-hundred different types of space debris shielding, yet still executes debris avoidance procedures [39]. 
2

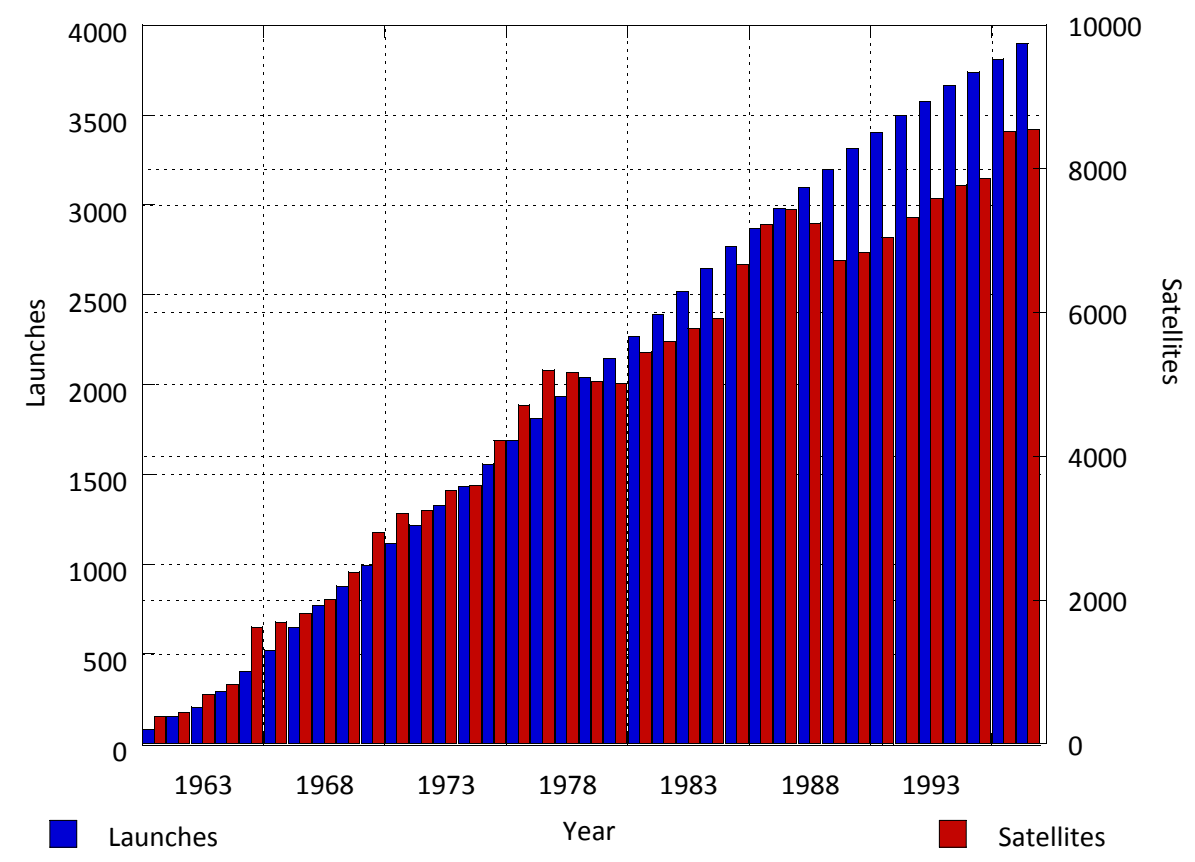

Figure 1.0.1: Cumulative end-of-year launches reaching earth's orbit or beyond (Left) and cumulative end-of-year satellite objects in orbit (Right) from 1957 to 1994 [41]

\subsection{Definition}

While many similar definitions of hypervelocity exist in literature, the following definition is adopted for the purposes of this study:

impact velocities on the order of or greater than the colliding material wave speeds.

In this regard, it is a regime initiated at a point when inertial stresses begin to outweigh the material strength. For some materials this can be considered speeds in excess of about 1 to $2 \mathrm{~km} / \mathrm{s}$ (2200 to $4500 \mathrm{mph}$ ). The lowest end of the investigated velocities, between about 1 to $3 \mathrm{~km} / \mathrm{s}$, is often considered the traditional ballistic regime since conventional military artillery and weapons, such as tank cannons and long-distance rifles, shoot within this range [4]. In the context of space assets, hypervelocity is often defined in terms of speeds greater than the escape velocity of the vehicle. On average, space debris collisions occur at $10 \mathrm{~km} / \mathrm{s}$ and micrometeoroid impacts at $20 \mathrm{~km} / \mathrm{s} \mathrm{[5].}$ 


\subsection{Motivation}

All earth orbits have particular mission-based advantages and consequently an abundance of orbiting space assets. While LEO, 200 to $2000 \mathrm{~km}$ above earth's surface, is commonly used to access large masses for piloted spacecraft, it also has the advantage of being the best range for automated highresolution imaging space vehicles and high-signal-strength communication satellites. Navigation systems such as the Global Position System, GPS, and communication assets typically reside in SEO between 10000 to $20000 \mathrm{~km}$ above earth's surface. Lastly GEO, 20000 to $36000 \mathrm{~km}$ above earth's surface, is most often used for weather satellites and a majority of telecommunication industry satellites [41].

\section{Space Debris}

In general, the farther away the debris from earth within the gravitational pull, the longer it will persist in orbit. In LEO, atmospheric drag will eventually cleanse the debris from orbit, however this may not happen for centuries depending on the size and location of the debris [41]. In early 2009 when an abandoned Russian military satellite collided with a working U.S. Iridium commercial satellite $800 \mathrm{~km}$ above earth's surface, the unknown amount of debris generated from that crash is predicted to remain in orbit for the next 10000 years [24]. While GEO contains far fewer space debris than LEO, it is predominantly solar radiation and solar radiation drag, not atmospheric drag, that decays the debris orbit over time. Regardless, GEO space debris between $1 \mathrm{~cm}$ and $1 \mathrm{~mm}$ in diameter have an estimated orbital life span of 60000 years [41]. Figure 1.2.1 catagorizes earth's orbiting space population as of 1989 , emphasizing that functional space assets maintain $6 \%$ of the total orbiting population.

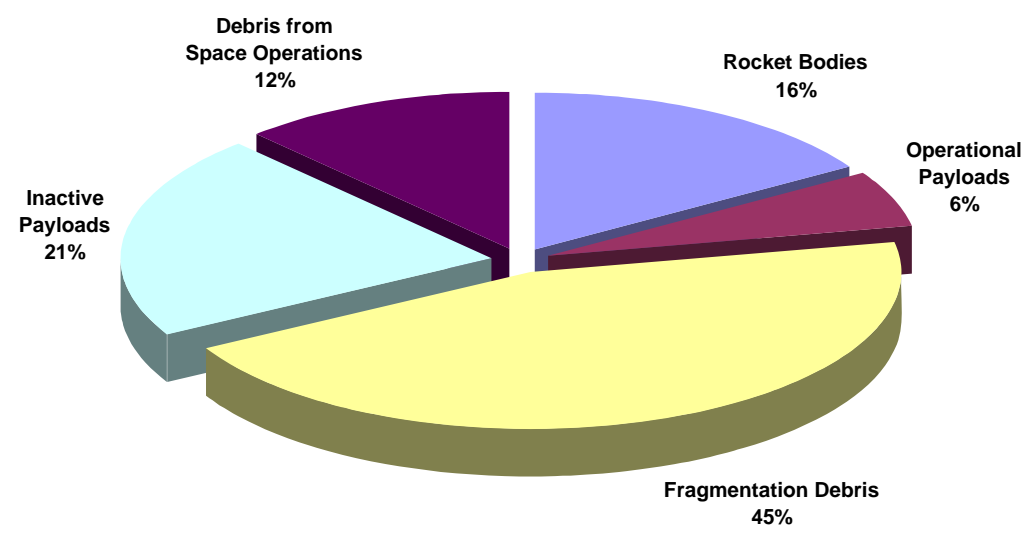

Figure 1.2.1: Cataloged near earth orbiting population from December 8, 1989 [50] 
Prior to 1990, no statistically significant measurements of debris environments for particles less than $10 \mathrm{~cm}$ in diameter existed [49]. While the main concern for space debris is its potential to compromise or degrade the structural integrity of a space vehicle, subsystem functionality such as electrical, optical, or thermal components are also equally threatened. Consequently, particles at micron and sub-micron diameters still pose a serious threat due to shock-induced melting, erosion, and plasma formation generated during a hypervelocity strike [13]. Currently the United States relies on the Orbital Debris Program Office at the NASA Johnson Space Center, and based on the US Space Command (formerly NORAD, the North American Aerospace Defense Command) to catalog debris, predict its development, and formulate mitigation policy for the environment from LEO to GEO [49]. While there are an estimated 8500 pieces of tracked space debris in earth's orbit, there are an estimated over 50000 pieces of untracked debris $1 \mathrm{~cm}$ in diameter or greater, and estimated trillions more untracked less than $1 \mathrm{~cm}$ in diameter [41]. As a result, the most potentially dangerous space debris is the smaller size debris (less than $1 \mathrm{~cm}$ ) due to its population density and unknown location at any given time.

Smaller debris impacts may not catastrophically fail a space asset, yet they can still do considerable damage, particularly when accumulated over time. For example, pressurized modules such as an external tank or crew capsule can burst when penetration from a hypervelocity impact is greater than $1 / 4$ of the wall thickness, and cables or tethers can snap when the diameters are reduced by $1 / 3$ of the original thickness [8]. In the case of a long single-strand space tether, this would correspond to a lifetime on the order of a few weeks to a few months. If a space asset requires solar cells for energy generation, even micron-sized hypervelocity impacts can create conducting paths that can lead to a short circuit and loss of functionality. To provide some perspective, each $8 \mathrm{~cm}^{2}$ solar cell on the Hubble Space Telescope which orbits $560 \mathrm{~km}$ above earth at approximately $7.5 \mathrm{~km} / \mathrm{s}$, experienced 12 micometeoroid or space debris impacts in 8.25 years, on average [8]. Table 1.1 summarizes various damage concerns and potential effects on vehicle performance while Figure 1.2.2 provides an illustration of the escalating "garbage collection" in earth's orbit. 

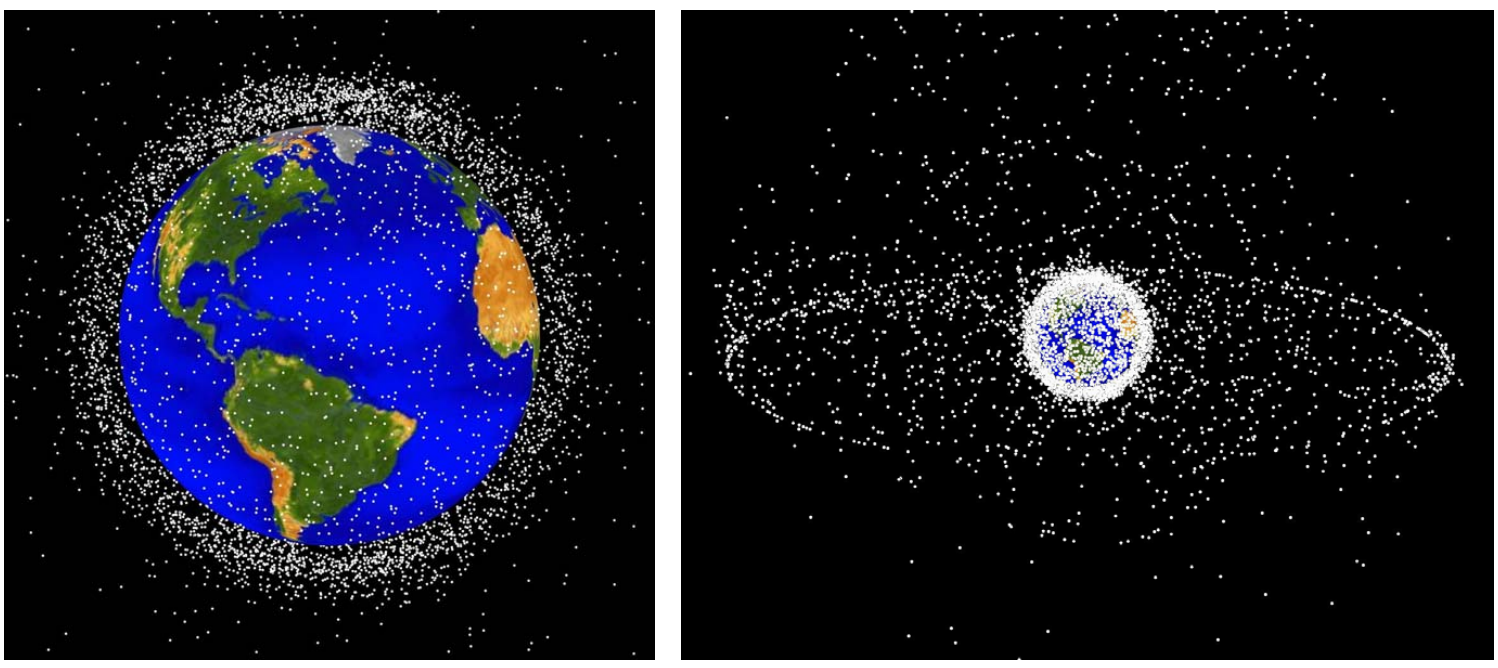

Figure 1.2.2: (Left) LEO orbital population image, (Right) GEO orbital population image, from NASA's Orbital Debris Program Office in 2010, where roughly $5 \%$ of the total visible white population dots are functional orbiting assets [1]
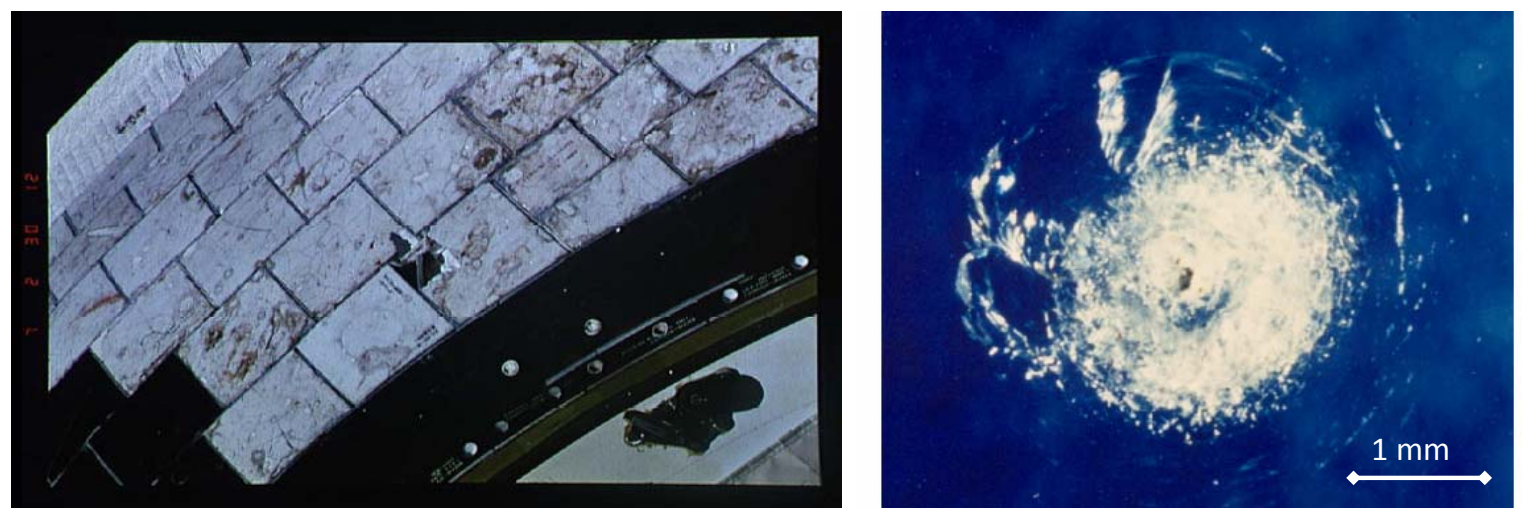

Figure 1.2.3: (Left) space shuttle Endeavour impact tile damage on Orbital Maneuvering System (OMS), image from NASA [31]. (Right) Window damage from hypervelocity impact received during STS-7 mission on space shuttle Challenger, image from Drolshagen [8] 
Table 1.1: Potential adverse effects of hypervelocity impacts on space assets [8]

\section{Cratering and Ejecta}

- surface erosion and degradation

· degradation of sensors, mirrors, internal instruments (secondary ejecta)

- degradation of windows

· sealing problems of exposed hatches, pressure changes

\section{Structural Damage}

· penetration of outer walls, damage of inner subsystems

- penetration of pressurized vessels (balloons, tanks, coolant loops, manned modules)

- cutting of cables or tethers

- short circuits

- damage to exposed focal plane arrays like CCDs

- catastrophic structural collapse, complete failure of space vehicle

\section{Plasma Effects}

- electrical interference

- current flow

- triggering of electrostatic discharges

- light flashes

\section{Momentum Transfer}

- change of attitude

- loss of target lock

- loss of formation flying

\section{Micrometeoroids}

A complete understanding of the origin and nature of the interplanetary dust cloud has yet to be determined, however over the years of space exploration, spacecraft measurements of cosmic dust flux have found that meteoroid flux is a function of meteoroid mass at 1 AU [52]. These high-velocity dust particles in the solar system contribute to the unknown and relatively untraceable components comprising of micrometeoroids. While characteristic micrometeoroids of cometary origin typically consist of iron-carbon, averaging $1 \mathrm{~mm}$ in diameter and $3 \mathrm{mg}$ in mass, these pieces also average ultra-hypervelocity speeds of $70 \mathrm{~km} / \mathrm{s}$ [14]. When ideas of space exploration began to come to fruition, scientists overestimated the threat from meteoroids. Fred Whipple, an astronomer who is 
credited for the Whipple Bumper in $1947^{1}$, predicted that 1 in 25 moonships would be destroyed by micrometeoroids. This concern was further strengthened when Sputnik 3 and Explore 3 satellites returned erroneous signals interpreted as meteoroid flux much higher than expected in the late 1950s [41]. Only later from data received on the Mercury, Gemini, and Apollo programs did the threat of micrometeoroid impact turn out to be much less severe than first perceived. Regardless, it is still present in some form, with the Ulysses and Galileo spacecraft having determined that a $1.5 \times 10^{-4}$ $\mathrm{m}^{-2} \mathrm{~s}^{-1}$ flux of interstellar grain with a mean mass of approximately $3 \times 10^{16} \mathrm{~kg}$ is passing through the solar system [52]. Table 1.2 lists the meteoroid penetration study results from various space science satellite studies, and Table 1.3 lists space assets affected by micrometeoroid and space debris collisions.

Table 1.2: Hypervelocity impact penetration data from ten space science satellites, data from Zook $[52]$

\begin{tabular}{lccc}
\hline & & \multicolumn{2}{c}{ Penetration Rate $\mathrm{m}^{-2} \mathrm{~s}^{-1}$} \\
\cline { 3 - 4 } Satellite & Thickness $(\mathrm{mm})$ & Shielded & Unshielded \\
\hline Explorer 16 & 25 & $3.85 \times 10^{-6}$ & $5.15 \times 10^{-6}$ \\
& 51 & $1.99 \times 10^{-6}$ & $2.66 \times 10^{-6}$ \\
Explorer 23 & 25 & $3.99 \times 10^{-6}$ & $6.09 \times 10^{-6}$ \\
Pegasus 1, 2, \& 3 & 51 & $2.37 \times 10^{-6}$ & $3.53 \times 10^{-6}$ \\
& 40 & $2.17 \times 10^{-6}$ & $3.16 \times 10^{-6}$ \\
& 200 & $2.43 \times 10^{-7}$ & $3.53 \times 10^{-7}$ \\
Lunar Orbiters 1-5 & 400 & $5.63 \times 10^{-8}$ & $8.17 \times 10^{-8}$ \\
\hline
\end{tabular}

\footnotetext{
${ }^{1}$ The Whipple Bumper is the most common shield design comprising of a light-weight metal plate suspended some distance from a backplate housing the structure of the space asset.
} 
Table 1.3: Table of spacecraft damaged by or suffering complete failure from space debris and meteoroid impact, data from Koons and Lai [10, 37]

\begin{tabular}{lccc}
\hline Vehicle & Date & Effect & Possible cause \\
\hline ISEE-1 & October 1977 & Detector window punctured & Meteoroid \\
ISEE-1 & August 1978 & All isobutane gas lost in 5 days & Meteoroid \\
Kosmos-1275 & July 1981 & Fragmented into 200 pieces & Space Debris \\
HST & April 1990 & 5000 impacts in 4 years & Meteoroid/Space Debris \\
Solar-A & August 1991 & Telescope punctured & Meteoroid \\
STS-45 & March 1992 & Gouges on wing edge & Space Debris \\
STS-49 & May 1992 & Chip in window pane & Space Debris \\
Olympus & August 1993 & Satellite failed to function & Meteoroid \\
SEDS 2 & March 1994 & Mission terminated & Meteoroid/Space Debris \\
MSTI 2 & March 1994 & Satellite communication lost & Meteoroid/Space Debris \\
\hline
\end{tabular}

\subsection{Impact Physics}

Numerous computational models are utilized for risk mitigation of hypervelocity impacts on every space endeavor [15], yet at the same time little is fully described in the numerical schemes regarding the mechanisms of damage evolution incurred from a hypervelocity strike. This lack of multi-phenomena damage evolution models for hypervelocity impacts is due to intense collision speeds which generate complex high-energy interactions including: melting, vaporization, dissociation, ionization, plasma formation, fracture, fragmentation, spallation, shear banding, mixed-phased flow, hydrodynamic instabilities, solid-solid phase transition, luminescence, and radiative transport, among others.

There is a distinct difference in the damage formation process from a hypervelocity impact on a thick structure (plane-strain conditions) versus a thin structure (plane-stress conditions), as well as a monolithic solid versus a layered media with interfaces. To pursue a discussion on the basic shock physics of a hypervelocity impact strike, and to correlate to the type of impacts investigated in later chapters, monolithic plane stress conditions are assumed. These high-speed impacts produce inelastic collisions which generate permanent deformation, as well as changes in both colliding bodies corresponding to the release or conversion of energy from relative motion into radiation and heat [42]. It is important to make a designation between the terms perforation and penetration when 
considering impacts of thin plates. In this study, perforation corresponds to the cases where the impactor has created a hole in the target plate at least the size of its characteristic length. Partial perforation occurs when the hole is less than the impactor characteristic length, and penetration is considered when the impactor has permanently deformed, or cratered, the target plate, but not necessarily passed through the entire thickness of the plate.

To descibe the physics of a hypervelocity impact, consider a simple two-dimensional model of a small piece of space debris striking a thin plate ${ }^{2}$ at initial velocity $v_{0}$. Further assume that the space debris is a right-circular cylinder (length equal to diameter) and impacts a thin semi-infinite plate at zero degrees obliquity as illustrated in Figure 1.3.1. Upon impact, two shock waves propagate away from the interface, $I$, one towards the end of the impactor, $\mathrm{S}_{1}$, and one towards the rear side of the plate, $S_{2}$. At nominally the same instant, rarefaction waves, $R_{1}$ and $R_{2}$, are transmitted toward the axis of symmetry of the impactor due to the fact that it has finite diameter. The formation of these rarefactions result in ejecta from the impactor and plate free surfaces. After the shock $\mathrm{S}_{2}$ has reached the rear surface of the plate, it is reflected as a rarefaction wave, $R_{3}$, to satisfy the zero pressure condition. Equivalently a rarefaction wave, $\mathrm{R}_{4}$, is reflected back from the impactor rear surface. It is important to note that because the initial shock compression is a non-isentropic process and the release of the rarefaction waves is an isentropic process, the entropy of the material is increased on impact and then brought back to ambient conditions adiabatically. Thus the additional energy is predominantly converted into localized heating at the impact site. If the shock strength is large enough, the excess entropy can lead to melting, vaporization, and plasma formation. Moreover, the rarefaction waves, $R_{1}, R_{2}, R_{3}$, and $R_{4}$, act as tension waves in the sense that fracture of the plate or impactor will occur if the net tensile stress at any point in either solid exceeds the fracture stress. As a result, additional rarefactions are generated at the free surface of the new fracture site to satisfy newly created stress free boundary conditions. This process continues until the strength of the rarefactions is less than the material strength. In this regard, the process of hypervelocity fracture at the impact site can be regarded as a multiple-spalling phenomenon initiated at the free surfaces [30].

\footnotetext{
${ }^{2}$ The terms debris, impactor, launch package, and projectile will be used interchangeably to describe the body performing the impact, as well as the terms shield, plate, and target as the body being impacted.
} 

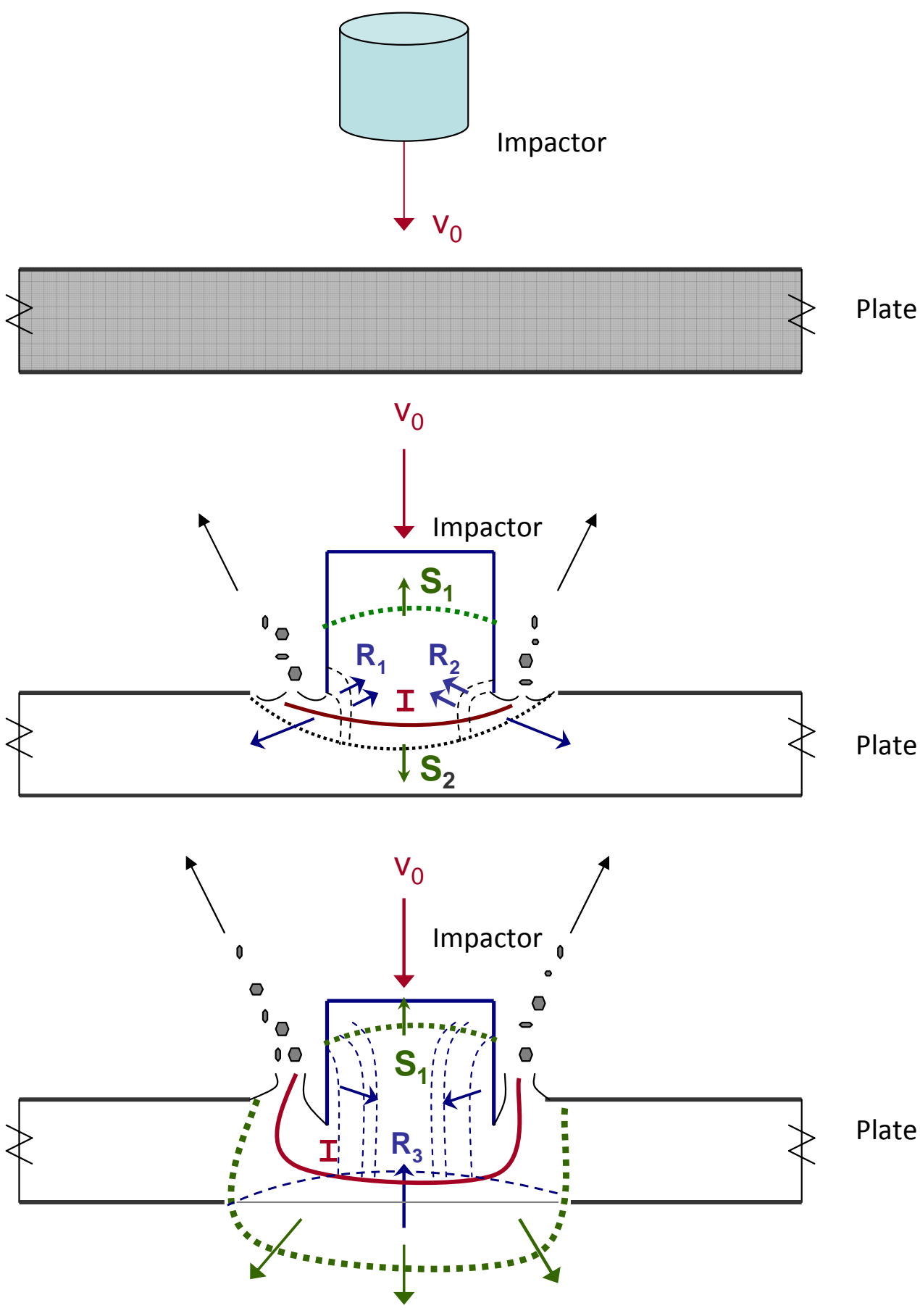

Figure 1.3.1: (Top) Simplified model of hypervelocity impact of right cylinder on semi-inifinite plate at initial velocity, $\mathrm{v}_{0}$, and $0^{\circ}$ obliquity. (Middle) Wave pattern estimated from directly after contact between impactor and plate, and (Bottom) a small time after initial impact in the case where the rarefaction wave has already reflected from rear surface of plate and permanent deformation can be seen to potentially lead to additional ejection of spall 
Given the following notation:

$$
\begin{aligned}
& \mathrm{v}_{0}=\text { impact velocity } \\
& \mathrm{v}_{1}=\text { velocity of material between shocks }\left(\mathrm{S}_{1} \text { and } \mathrm{S}_{2}\right) \\
& U_{1}=\text { velocity of shock } \mathrm{S}_{1} \text { in impactor } \\
& U_{2}=\text { velocity of shock } \mathrm{S}_{2} \text { in plate } \\
& P_{1}=\text { pressure in the region between shocks }\left(\mathrm{S}_{1} \text { and } \mathrm{S}_{2}\right) \\
& \rho_{0}=\text { density of uncompressed impactor material } \\
& \rho_{1}=\text { density of impactor material at pressure } \mathrm{P}_{1} \\
& q_{0}=\text { density of uncompressed plate material } \\
& q_{1}=\text { density of plate material at pressure } \mathrm{P}_{1},
\end{aligned}
$$

taking into account continuity across the interface, the normal shock relations from impact can be described as

$$
\begin{aligned}
P_{1} & =\frac{\rho_{0} \mathrm{v}_{0}^{2}}{1-\left(\frac{\rho_{0}}{\rho_{1}}\right)}\left[1+\left(\frac{\rho_{0}\left[1-\frac{q_{0}}{q_{1}}\right]}{q_{0}\left[1-\frac{\rho_{0}}{\rho_{1}}\right]}\right)^{\frac{1}{2}}\right]^{-2} \\
\mathrm{v}_{1} & =\mathrm{v}_{0}\left[1+\left(\frac{q_{0}\left[1-\frac{\rho_{0}}{\rho_{1}}\right]}{\rho_{0}\left[1-\frac{q_{0}}{q_{1}}\right]}\right)^{\frac{1}{2}}\right]^{-1} \\
U_{1} & =\frac{\mathrm{v}_{0}-\left(\frac{\rho_{1}}{\rho_{0}}\right) \mathrm{v}_{1}}{\left(\frac{\rho_{1}}{\rho_{0}}\right)-1} \\
U_{2} & =\frac{\mathrm{v}_{1}}{1-\left(\frac{q_{0}}{q_{1}}\right)} .
\end{aligned}
$$

In order to solve for velocities and pressures, specific impactor and plate material Hugoniot curves would be necessary and are empirically determined for most common engineering materials.

Figure 1.3.2 illustrates the impact process on a pressure-volume plot. Considering the case where the material of the impactor and plate have the same initial density, $\rho_{0}$, on contact these elements are consequently compressed to some extreme pressure, $P_{H}$, as shown on the Hugoniot curve (points 1-2). The material is returned to ambient conditions adiabatically by the release of rarefaction waves (points 2-3). The additional entropy created by this process heats the material, and the specific internal energy, $e_{H}$, of the shock-compressed material is equal to the area created underneath the dashed line of angle $P_{H}\left(\mathrm{v}_{0}-\mathrm{v}_{H}\right) / 2$, whereas the area under the adiabat corresponds to the energy returned on expansion by the material in pressure-volume work. Since the area under the adiabat is less than that of the dashed line, the difference is proportional to the residual heat left in the material elements. If the shock strength is high enough, the residual heat produces melting, and in some cases vaporization and super-heated vapor of the impactor or plate. This additional entropy also dictates the state and size of spallation or ejecta, from solid to super-heated vapor [30]. 


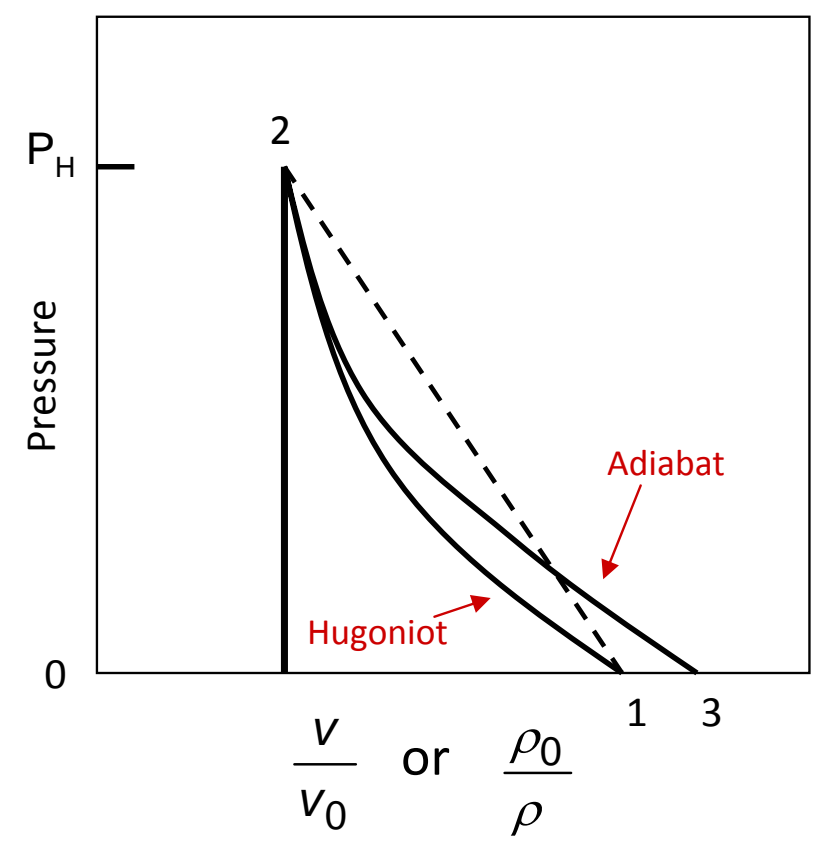

Figure 1.3.2: Hypervelocity impact process on a pressure-volume plot [30]

The flow of the initial shocks traveling towards the rear of the plate and the impactor are essentially one-dimensional until reached by the rarefaction waves generated at the free surfaces, as illustrated in Figure (1.3.3). If the impactor and plate are of the same material, no rarefaction wave would be generated at the interface [30].
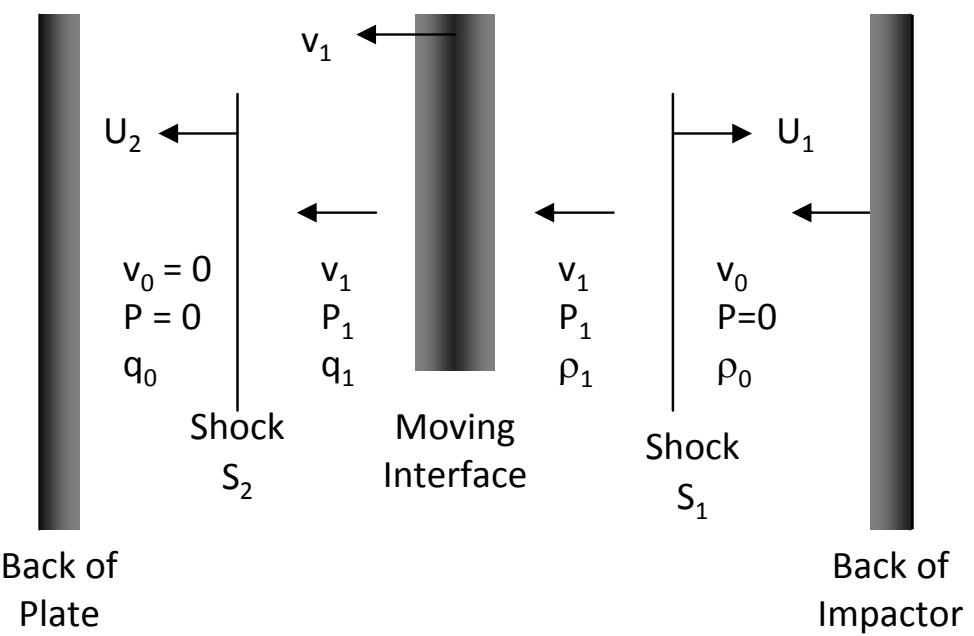

Figure 1.3.3: One-dimensional representation of the shocking and rarefaction wave process immediately after impact with coordinates fixed on the plate [30] 
Another means of illustrating impact wave progression is by an x-t diagram, as in Figure 1.3.4. Considering the length of impactor as $L$ and the target thickness of $\ell$, the rarefaction waves generated in the impact $\left(\mathrm{R}_{1}\right.$ and $\left.\mathrm{R}_{2}\right)$ can attenuate the shock $\left(\mathrm{S}_{1}\right)$. Consequently, the resulting wave pattern depends on specific geometry and material properties of the impactor and plate. For example, the case where $\ell$ is less than $L$ (or the plate is thinner than the length of the impactor), the cylindrical rarefaction waves can overtake the impact shock, $\mathrm{S}_{1}$, on the axis of symmetry of the impactor, if the error in assuming a one-dimensional approximation is small.

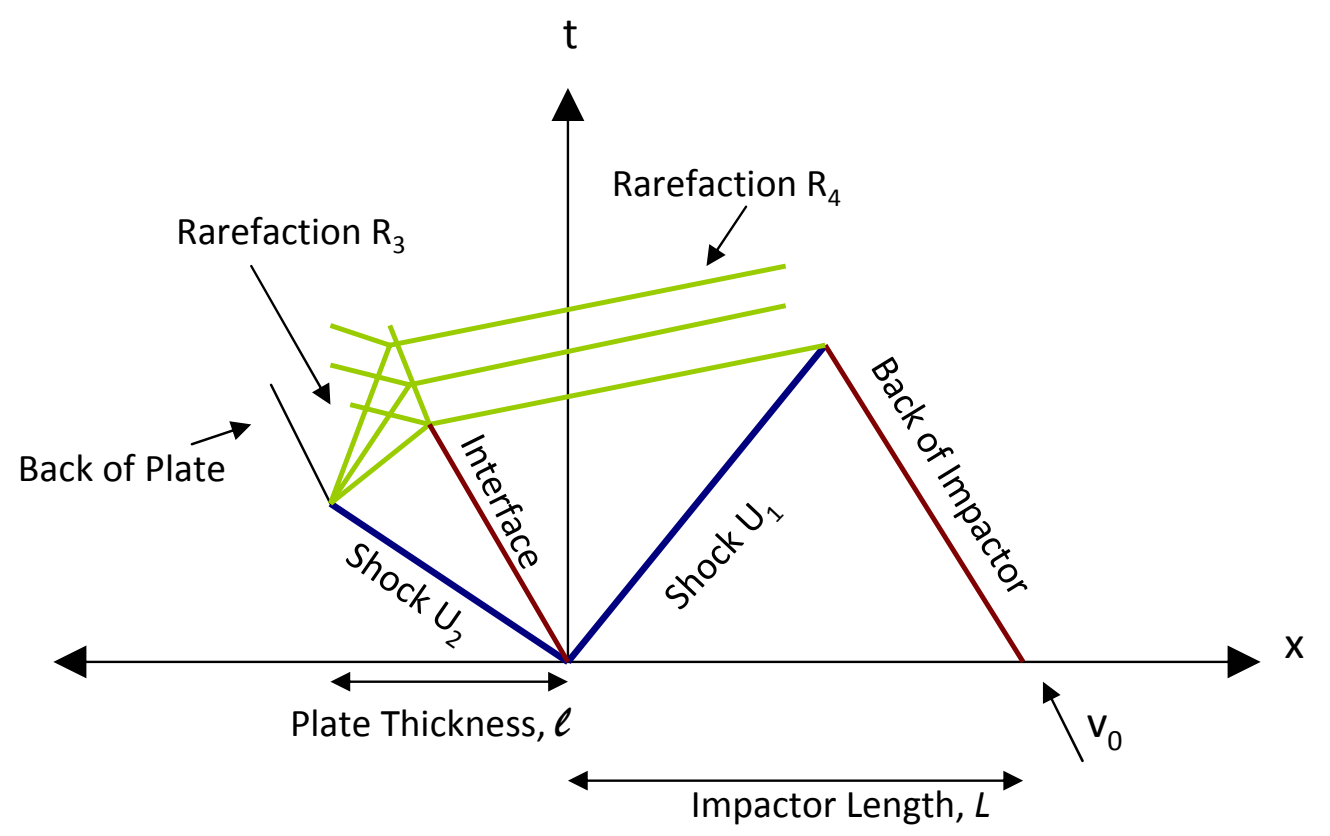

Figure 1.3.4: Time-distance (x-t) diagram illustrating hypervelocity impact shock and rarefaction process [30]

\subsection{History}

The first credited invention of the light-gas gun was by Dr. W.D. Crozier and his team at the New Mexico School of Mines (NMSM) in 1946, where using hydrogen gas as propellant, accelerations of light impactors were created up to $4 \mathrm{~km} / \mathrm{s}$ [40]. By the 1950s, national defense as well as space-related programs were examining means of creating hypervelocity. Unlike the space program concerned with damage to space assets from hypervelocity impacts, the defense effort for generating hypervelocity was motived by examining the possibility of knocking enemy missiles off target via bombardment of a large number of small, ultra-fast moving projectiles launched from a traditional satellite. One of the earliest efforts to develop experimental facilities to examine these high speeds with a single- 
strike precision was at the U.S. Naval Ordinance Test Station at China Lake in 1959 [35]. Shorlty after, Ames Aeronautical Laboratory (now NASA Ames) developed a launcher in the same manner as the Crozier gun, yet used helium, and could shoot up to $4.6 \mathrm{~km} / \mathrm{s}$. Two other methods to compress light-gas to higher pressures than the existing guns mentioned were developed at Ames. One technique required multiple shock compressions to heat and compress the gas to the desired firing pressure. This method included two stages, one where a light piston accelerated the light-gas enough to produce a driving shock to enter a second stage, where the shock would then compress using multiple reflections between the driver face and the transition section at the base of the projectile. The other method required a heavy piston with a deformable front section using an isentropic compression cycle. The deformable piston was extruded through a taper and into the second-stage launch tube. Other light-gas guns were also built and operated in the 1950s and 1960s including Canada's Armament Research and Development Establishment. McGill University built and operated two three-stage light-gas ranges in the 1960s [40]. Today operational hypervelocity gas guns in the United States can be found at Sandia, Lawrence Livermore, and Los Alamos National Laboratories, NASA Ames, Johnson, Glenn, and White Sands, a few private companies, and select universities. A comparison of a few gas-gun facilities is shown in Table 1.4.

Table 1.4: Hypervelocity impact conditions examined in various thin plate test databases [46]

\begin{tabular}{lccc}
\hline Gas-Gun Facility & Year & $\begin{array}{c}\text { Impact Velocity } \\
(\mathrm{km} / \mathrm{s})\end{array}$ & $\begin{array}{c}\text { Impactor Diameters } \\
(\mathrm{cm})\end{array}$ \\
\hline NASA Johnson Space Center & $1990 \mathrm{~s}$ & $2.50-8.06$ & $0.02-1.91$ \\
NASA Marshall Space Flight Center & $1980 \mathrm{~s}$ & $1.62-8.04$ & $0.32-1.27$ \\
Eglin Air Force Base & 1967 & $3.23-5.82$ & $0.32,0.64$ \\
Aerospace Group, Boeing Company & 1965 & $1.40-7.83$ & $0.16,0.32,0.64$ \\
NASA Johnson CR-915 & 1965 & $0.72-8.08$ & $0.16-0.48$ \\
General Motors Defense Research Laboratories & 1963 & $1.37-8.06$ & $0.32,0.48,0.64$ \\
\hline
\end{tabular}

\subsection{Test Facility}

A unique two-stage light-gas gun facility was used to experimentally investigate hypervelocity impacts. Originally designed by Southwest Research Institute and completed in 2006 [19], this particular gun is a joint venture between the California Institute of Technology and NASA's Jet Propulsion Laboratory (JPL), known as the Small Particle Hypervelocity Impact Range (SPHIR). Launch capa- 
bility with slug ${ }^{3}$ impactor technology includes impact velocities between approximately 1 and $7 \mathrm{~km} / \mathrm{s}$ having masses between 5 to $50 \mathrm{mg}$ of polymer or metal composition. Faster impactor speeds are currently being investigated using sabot technology. Launch packages in all tests conducted had a diameter of $1.8 \mathrm{~mm}$, with either spherical or cylindrical geometry. The facility itself is approximately $6 \mathrm{~m}$ in total length with a smooth-bore design, flight tube length of $3 \mathrm{~m}$, and a $2 \mathrm{~m} \times 1 \mathrm{~m} \times 1 \mathrm{~m}$ target chamber housing over a dozen optical ports. The envelope of functional kinetic energies and kinetic energy densities is shown in Figures 1.5.2 and 1.5.3.

\footnotetext{
${ }^{3}$ Slug refers to impactors where the entire loaded projectile collides with the target, whereas sabot refer to a launch package that a portion of the impactor is made to be stripped away during flight, typically by a radial force component on a rifled bore design.
} 


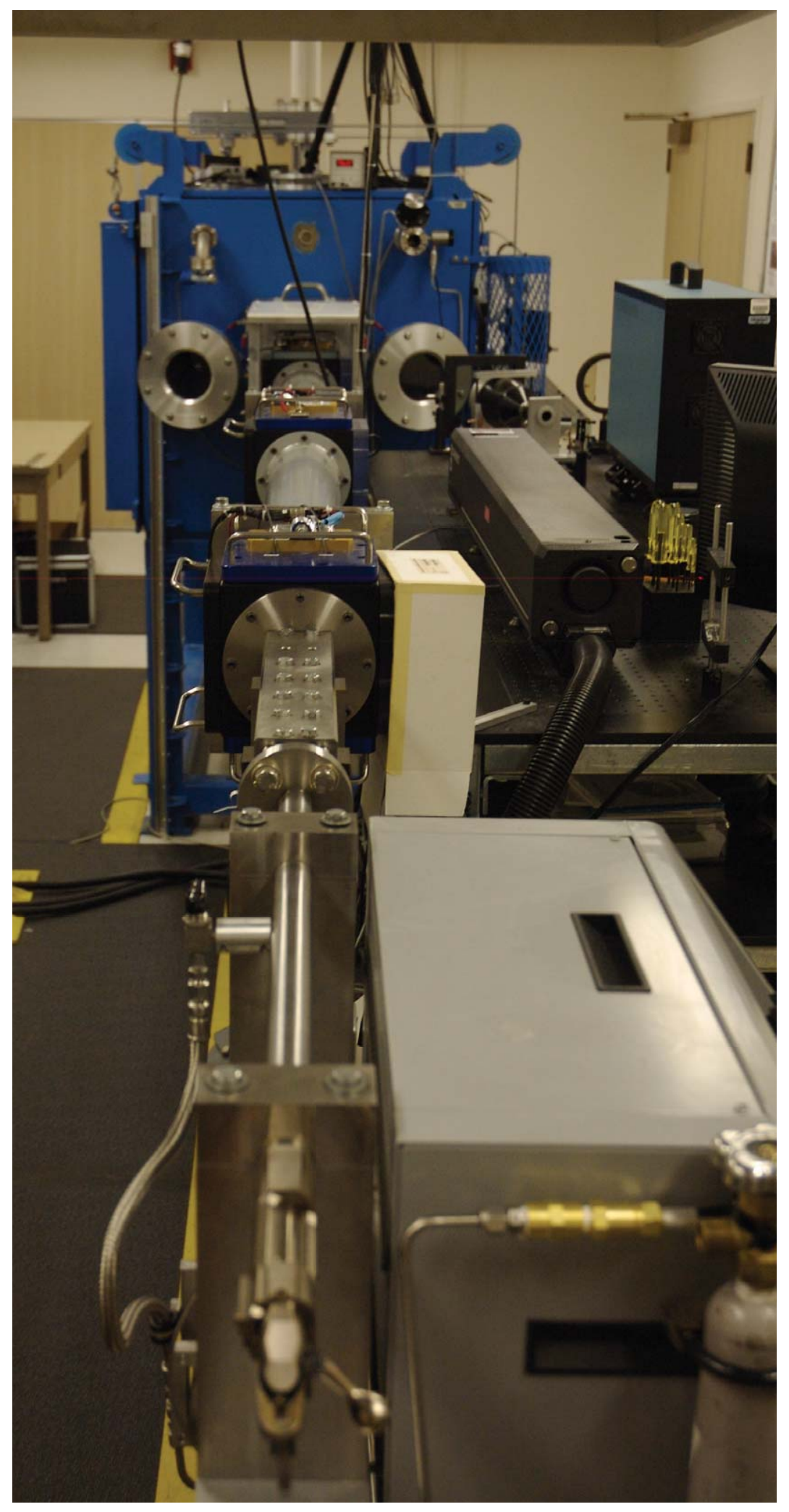

Figure 1.5.1: Two-stage light-gas gun facility viewed down the shot line from trigger pull to target tank 


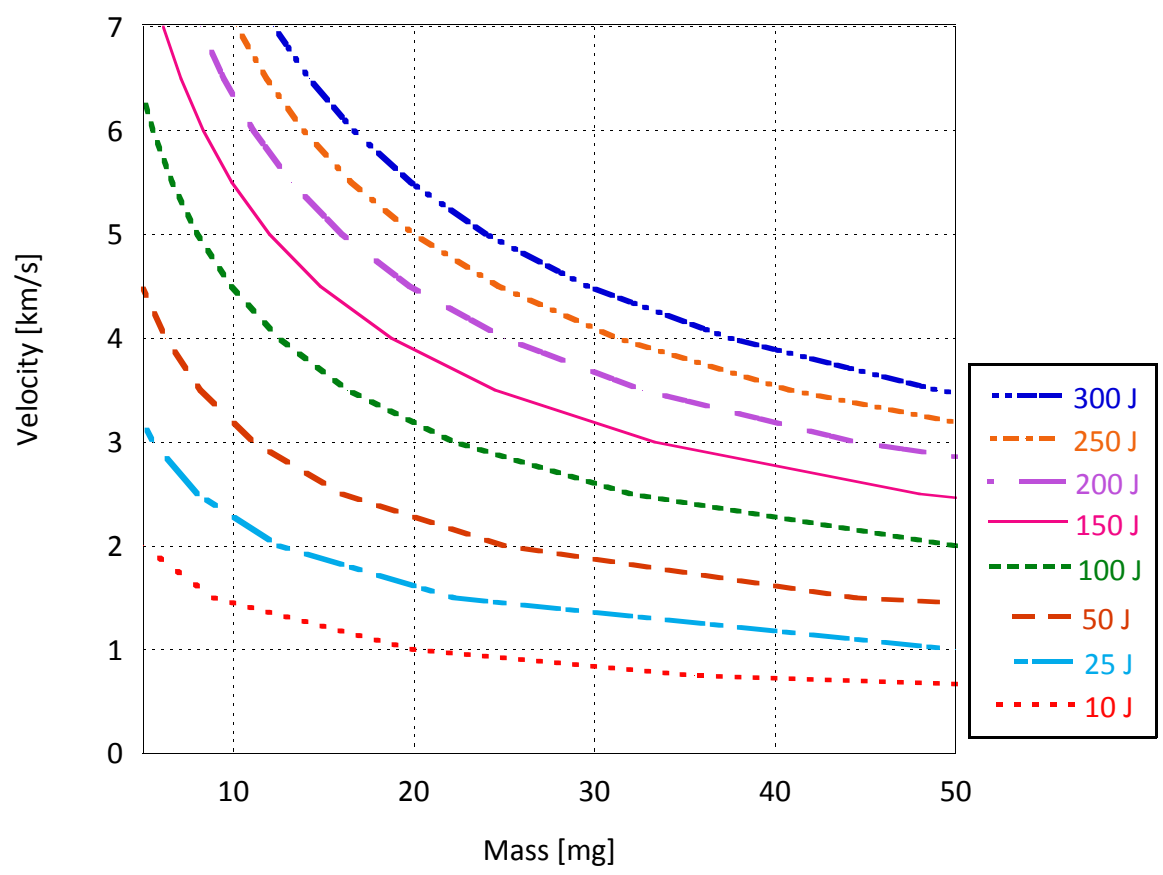

Figure 1.5.2: Working envelope of two-stage light-gas gun impactor velocity and mass for certain constant kinetic energy curves

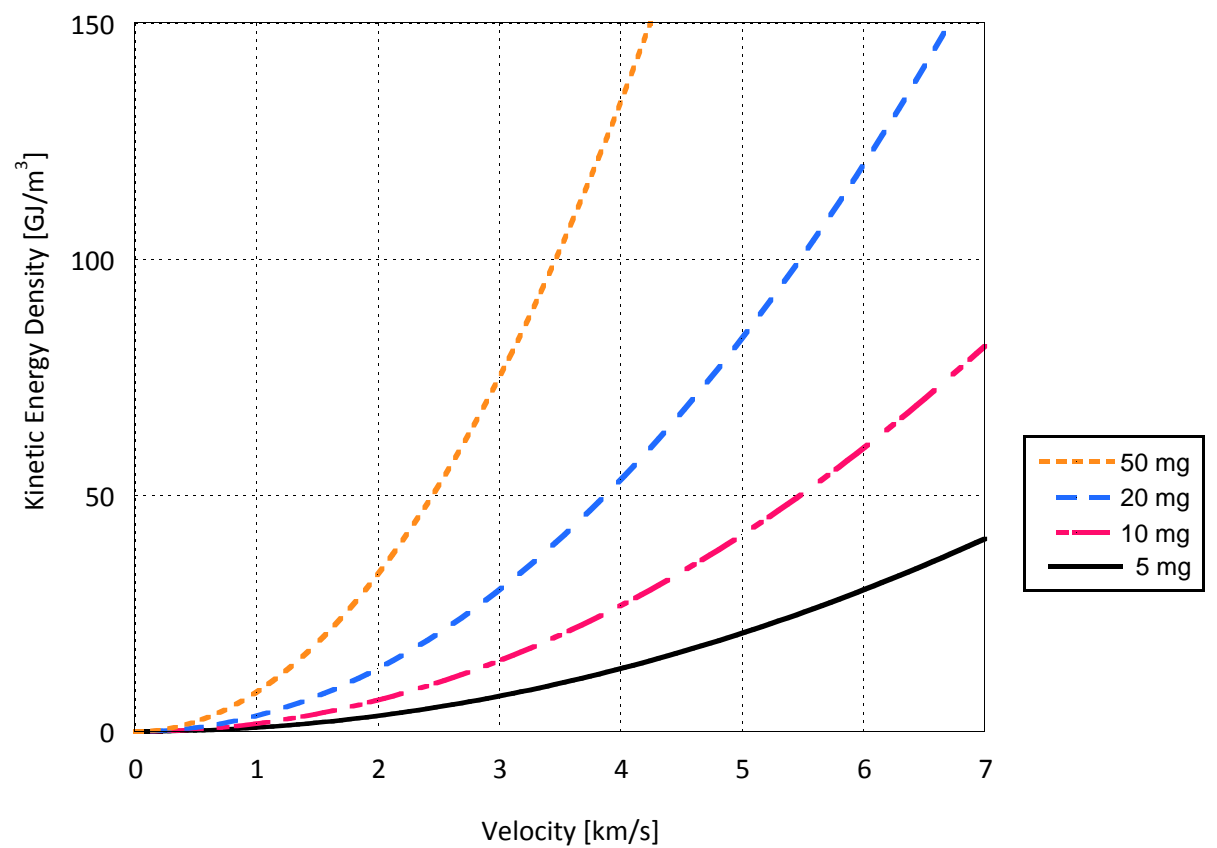

Figure 1.5.3: Working envelope of two-stage light-gas gun kinetic energy density for usable span of impactor mass, assuming a $1.8 \mathrm{~mm}$ diameter sphere volume 


\subsection{Launch Mechanics}

The two-stage light-gas gun consists of a breech, pump tube, accelerated reservoir (AR) section, launch tube, flight tube, and target tank as shown in Figure 1.6.1. The firing mechanism is initiated by a Sako 22-250 rifle action where an empty rifle cartridge is primed, packed with 0.9 grams of smokeless gun powder and a lowlint tissue wad of approximately $55 \mathrm{mg}$, and placed in the breech. A deformable high density polyethylene (HDPE) piston is inserted into the uprange end of the pump tube, otherwise known as stage 1 of the two-stage light-gas gun. The impactor is inserted into the uprange end of the launch tube, known as stage 2, with a $75 \mu \mathrm{m}$ thick Mylar disc placed between the AR section and the launch tube. All portions of the gun are held in place by high-strength steel restraints. Prior to testing, every section downrange of the breech is evacuated and the pump tube is filled with a predetermined amount of light-gas. The lower the molecular weight hence 'light-gas', the higher the speed of sound in the medium. Consequently, all tests in this study use hydrogen gas in the pump tube. To launch the impactor, the gun powder charge is ignited by the trigger pull mechanism which converts the chemical energy of the primer explosion initiated when hit by the firing pin, to kinetic energy setting the HDPE piston (approximately $5.6 \mathrm{~mm}$ diameter) into motion inside the pump tube. This event compresses the hydrogen gas inside the pump tube, generating a high energy shock front. The shock front then reaches the AR section, which is essentially a small converging nozzle that tapers from $5.6 \mathrm{~mm}$ to $1.6 \mathrm{~mm}$ in diameter. The flow is further accelerated through the volume change of the AR nozzle shape, and the piston gets extruded and stuck inside the AR section. The shock front passes through the AR ahead of the piston and reaches the thin Mylar disc on the downrange side of AR section. The disc is then burst by the shock front, similar to firing mechanisms of a conventional shock tube, with a punctured hole roughly the diameter of the converging section of the AR. The focused shock is then released uniformly onto the impactor that is loaded just inside the launch tube on the other side of the Mylar disc. The impactor is accelerated thorough the launch tube, enters free flight in the flight tube, and strikes the target in the target chamber. The evacuated chambers have a working range of 0.13 to $2.4 \mathrm{kPa}$ (1 to 18 Torr) of atmosphere present during a test. 


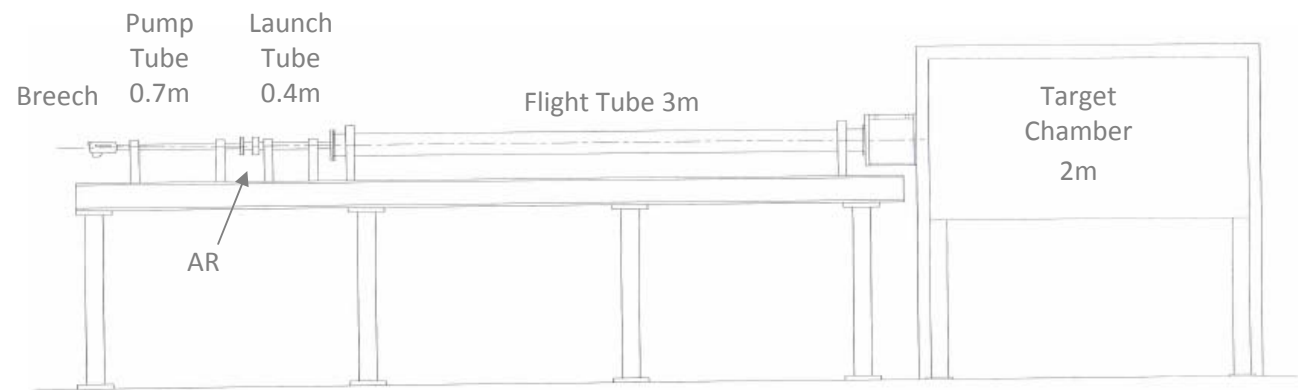

Figure 1.6.1: Schematic of the two-stage light-gas gun viewed directly from the side [19]

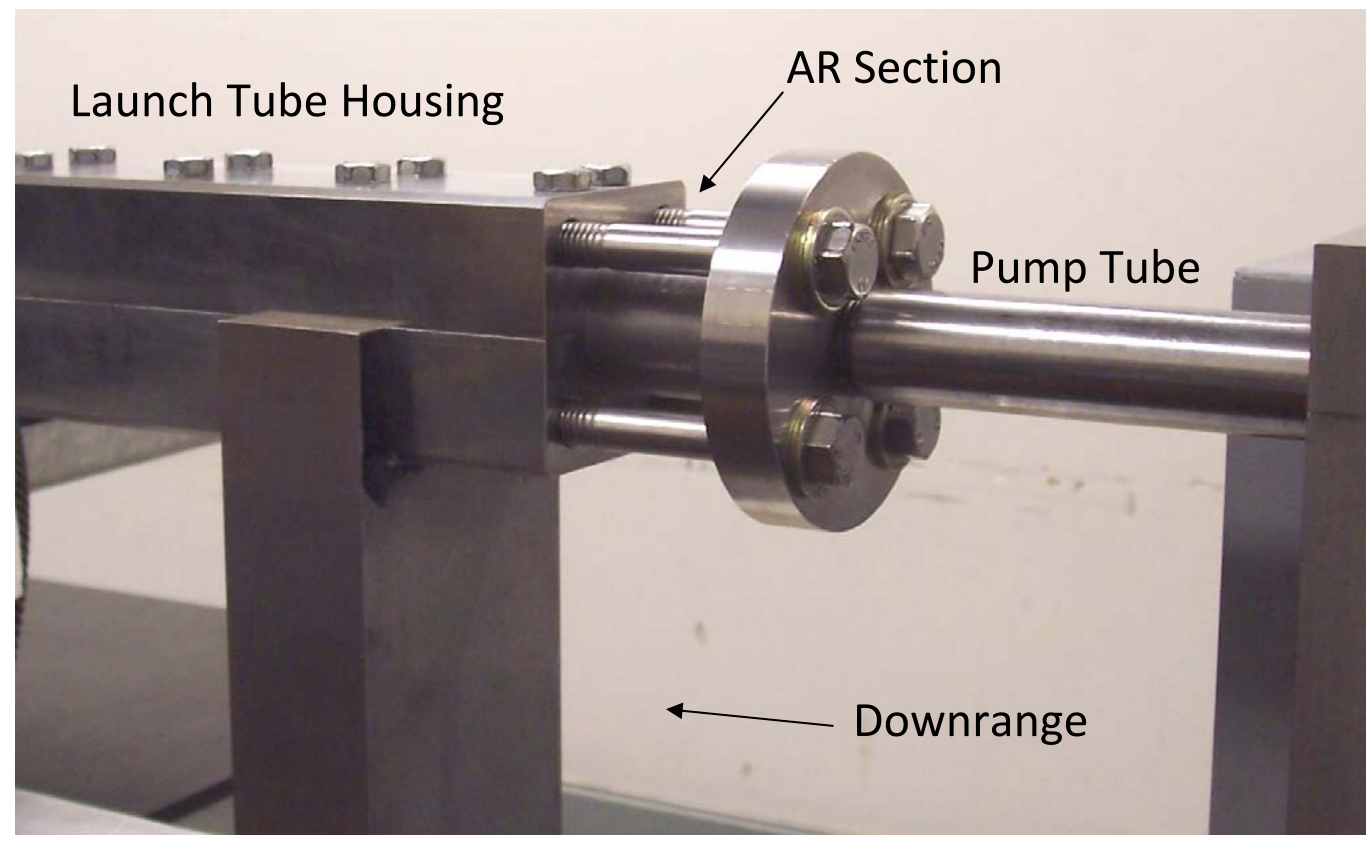

Figure 1.6.2: Photograph illustrating AR separation between stage 1 and stage 2 of the two-stage light-gas gun used for experiments [12] 


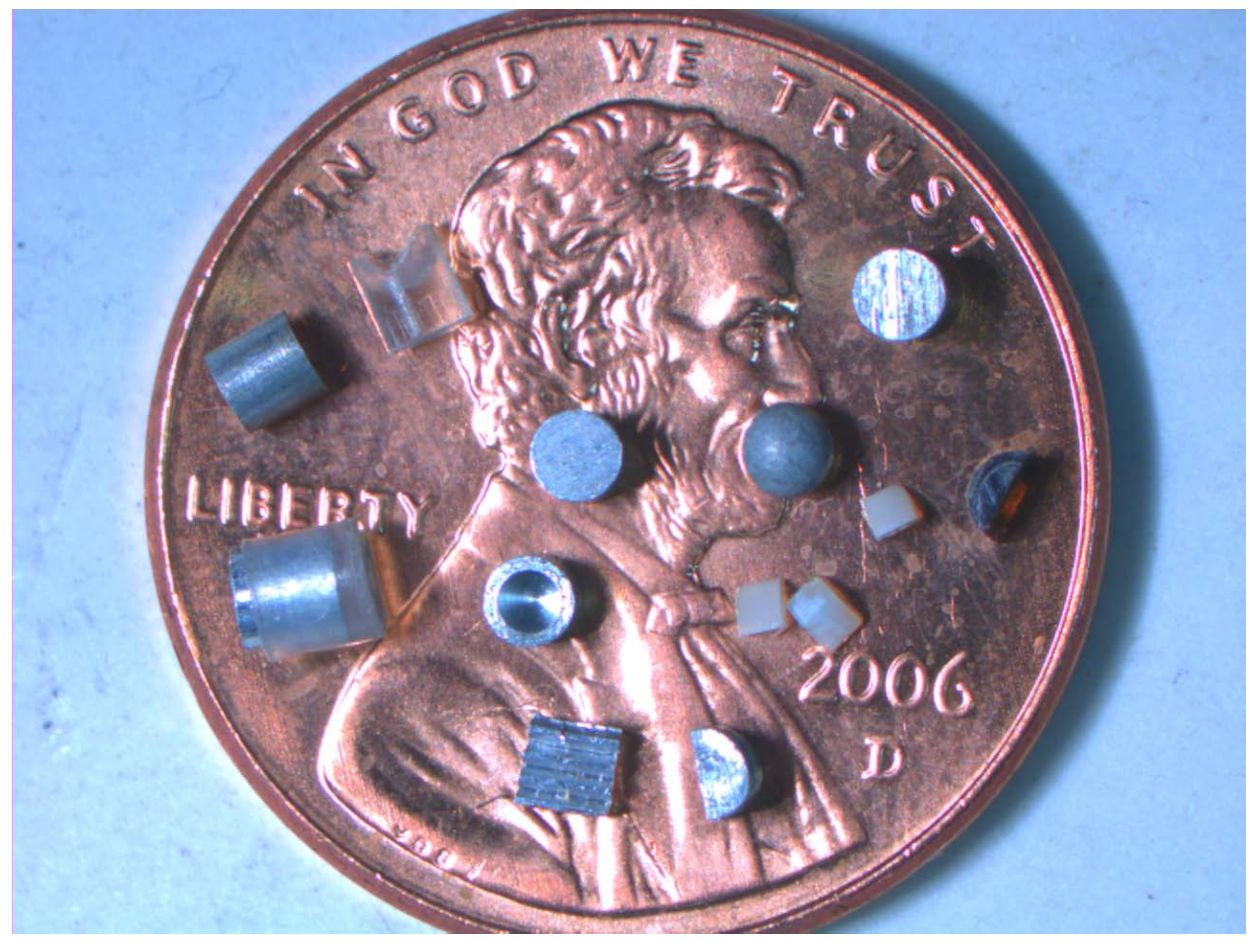

Figure 1.6.3: Example spherical and cylindrical aluminum, steel, polycarbonate, and nylon impactors, and sectioned impactors on a penny for scaling perspective

\subsection{Facility Capability}

The facility was designed with enhanced flexibility in the range of materials that could be used as impactors, as well as the size and type of structures that could be configured in the target chamber, varying shot obliquity, and diagnostics. To date, impactor materials tested include steel, aluminum, tantalum, nylon 6-6 $6^{4}$, and polycarbonate, and target materials have ranged from metals and polymers to ceramic composites and foams for various mission-based NASA applications. Due to the current configuration and safety limits of the components, the light-gas pressure cannot exceed $2 \mathrm{MPa}$ (300 psi) [19], and the limit of the vacuum pump capability is approximately $135 \mathrm{~Pa}$ (1 Torr). A few images of the diverse capability of the facility are shown in Figures 1.7.1 to 1.7.5.

\footnotetext{
${ }^{4}$ Nylon 6-6 is the most common form of nylon made from hexamethylene diamine and adipic acid, which gives 12 carbon atoms, or 6-6 as the name suggests.
} 

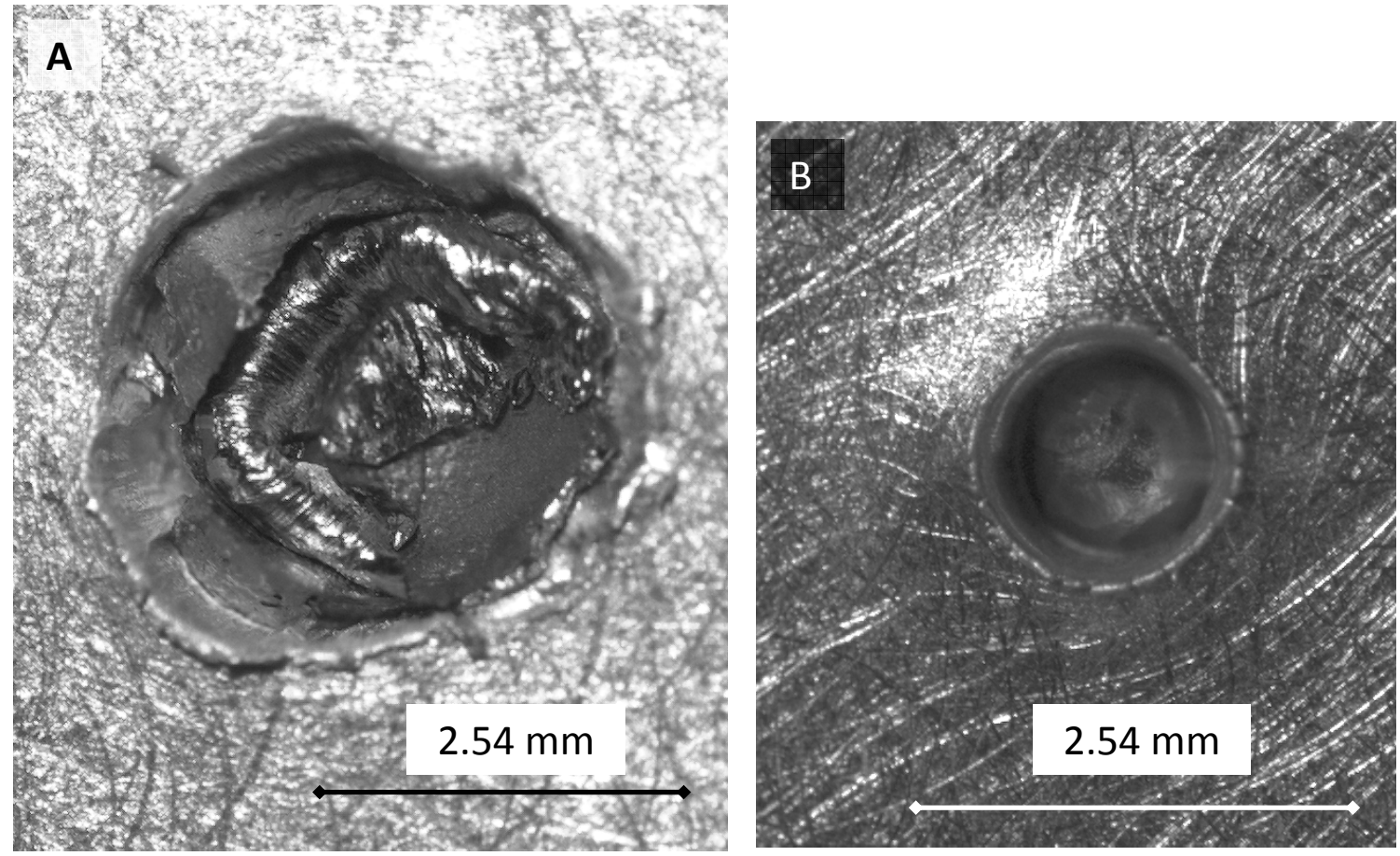

Figure 1.7.1: (A) Tantalum sphere, $1.8 \mathrm{~mm}$ diameter, embedded into 304 stainless steel plate $150 \mathrm{~mm} \times 150 \mathrm{~mm} \times 2.3 \mathrm{~mm}$ upon impact at $1.46 \mathrm{~km} / \mathrm{s}, 0^{\circ}$ obliquity.

(B) Tantalum sphere (1 mm diameter) from sabot technology development embedded into Aluminum 6061-T6 plate $150 \mathrm{~mm} \times 150 \mathrm{~mm} \times 25 \mathrm{~mm}$ upon impact at $3 \mathrm{~km} / \mathrm{s}, 0^{\circ}$ obliquity 

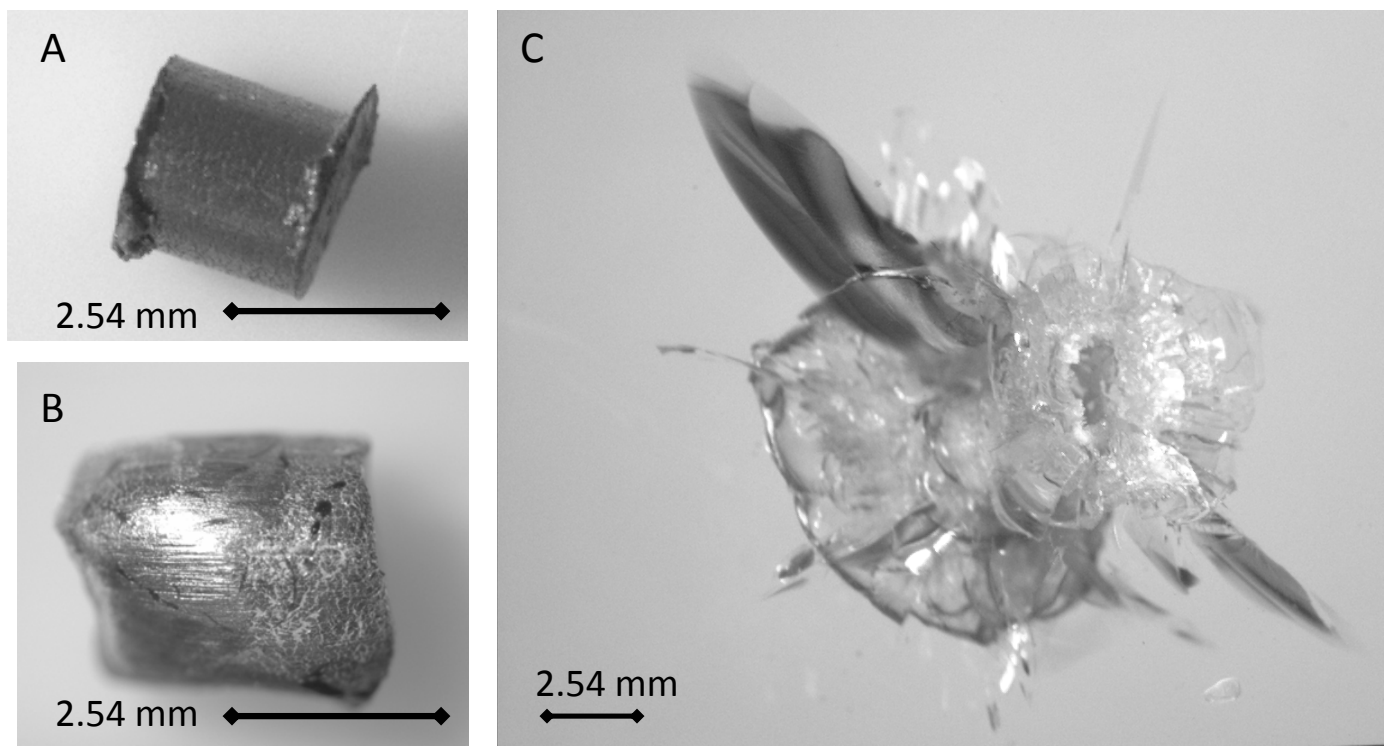

Figure 1.7.2: (A) Tantalum right cylindrical slug, $1.8 \mathrm{~mm}$ and length, before impact. (B) Tantalum right cylindrical slug after impact with Homalite 100 plate, $150 \mathrm{~mm}$ diameter and $2.3 \mathrm{~mm}$ thick, at $0^{\circ}$ obliquity at $1.5 \mathrm{~km} / \mathrm{s}$. (C) Homalite 100 plate impact site, isometric view

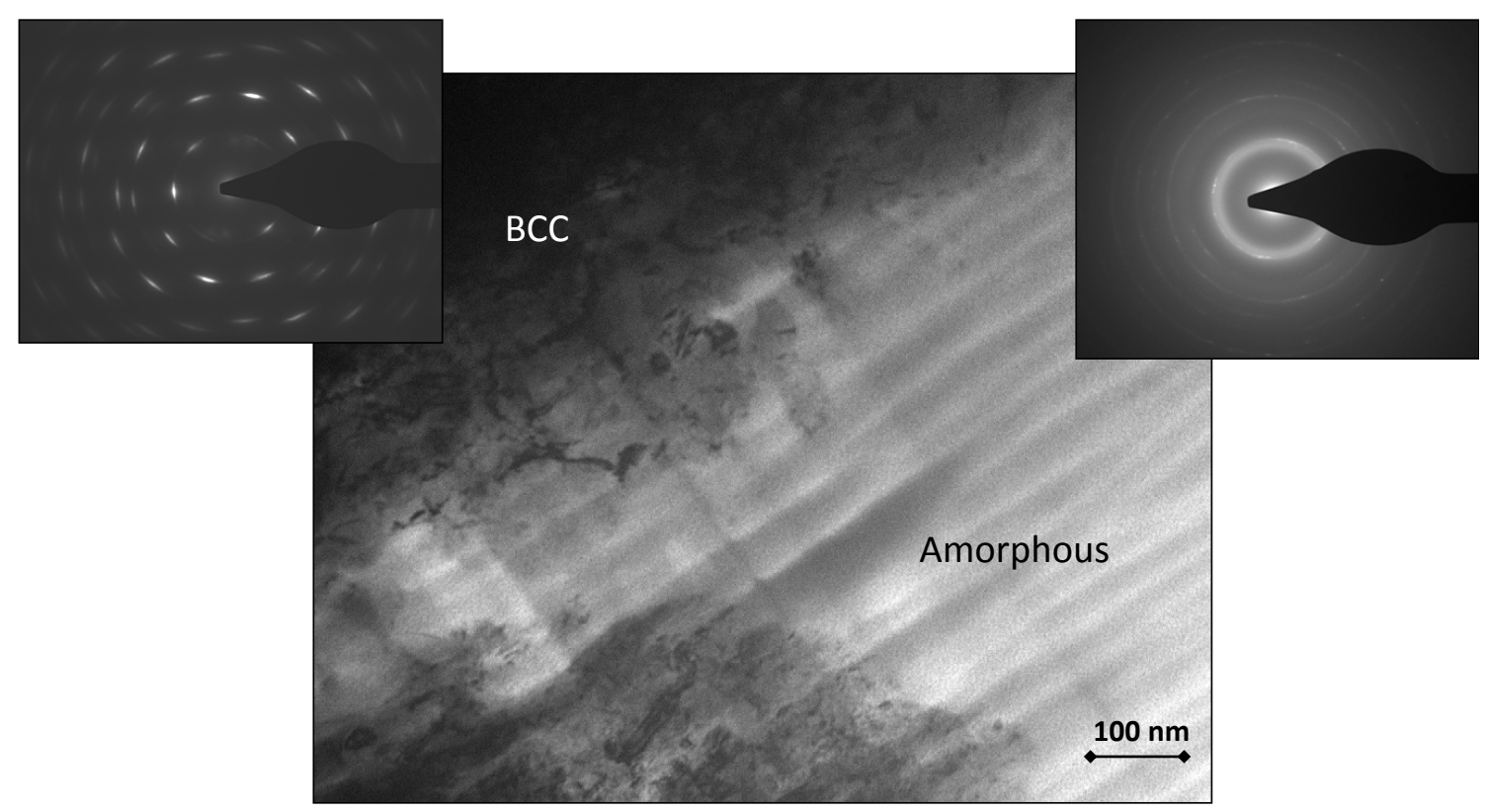

Figure 1.7.3: Bright-field TEM image of impactor (B) from Figure 1.7.2, a tantalum slug after being impacted against Homalite 100 plate, $150 \mathrm{~mm}$ diameter and $2.3 \mathrm{~mm}$ thick at $0^{\circ}$ obliquity and $1.5 \mathrm{~km} / \mathrm{s}$. Image shows region interface between two phases of the tantalum, $\mathrm{BCC}$ and amorphous glass-like, with diffraction patterns inset. Image credit: Carol Garland, Caltech 2008 

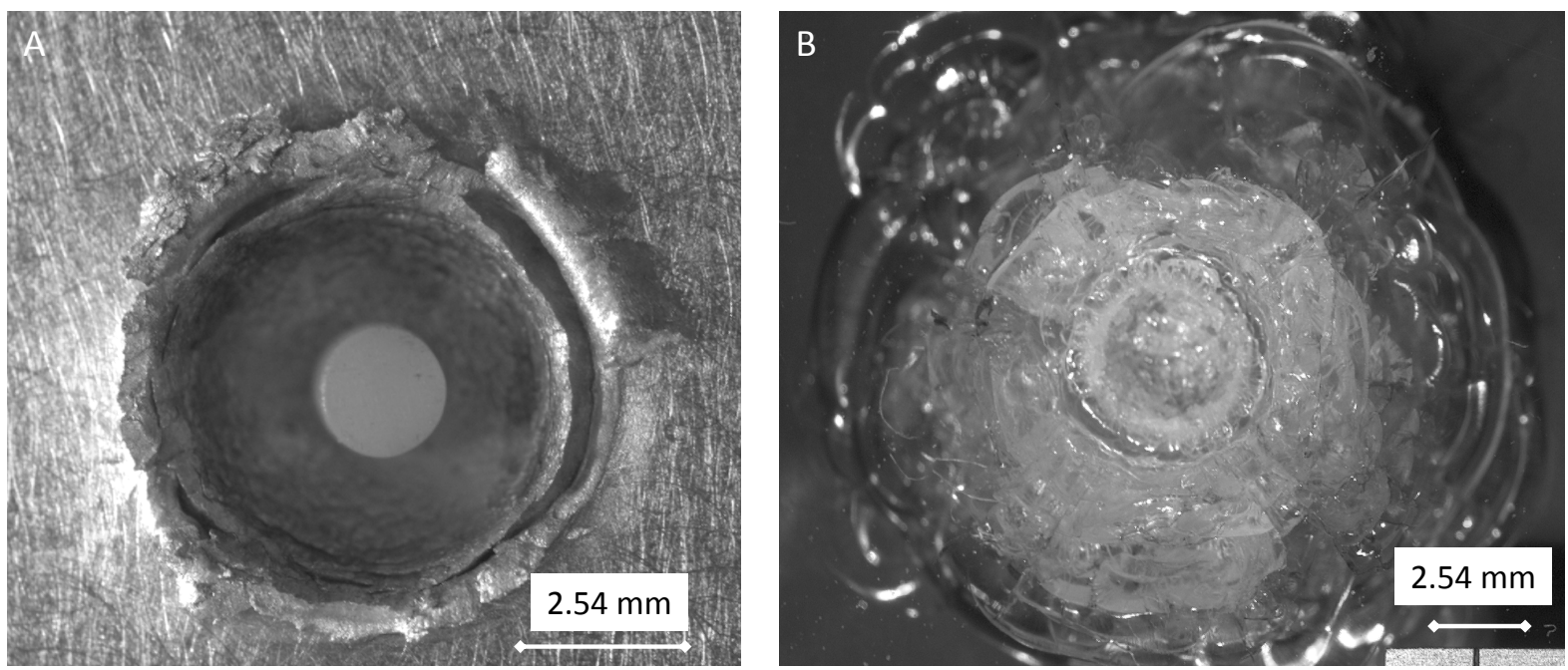

Figure 1.7.4: Nylon right cylindrical slug, $1.8 \mathrm{~mm}$ in length and diameter, having impacted Aluminum 6061-T6 plate $150 \mathrm{~mm} \times 150 \mathrm{~mm} \times 25 \mathrm{~mm}$ at $6 \mathrm{~km} / \mathrm{s}, 0^{\circ}$ obliquity. (A) At this high speed, the impactor vaporizes, but for perspective, an un-tested identical projectile is placed inside the impact crater generated. (B) Homalite 100 plate, $150 \mathrm{~mm}$ diameter and $6 \mathrm{~mm}$ thick, having been impacted by nylon right cylindrical slug at $6.5 \mathrm{~km} / \mathrm{s}, 0^{\circ}$ obliquity
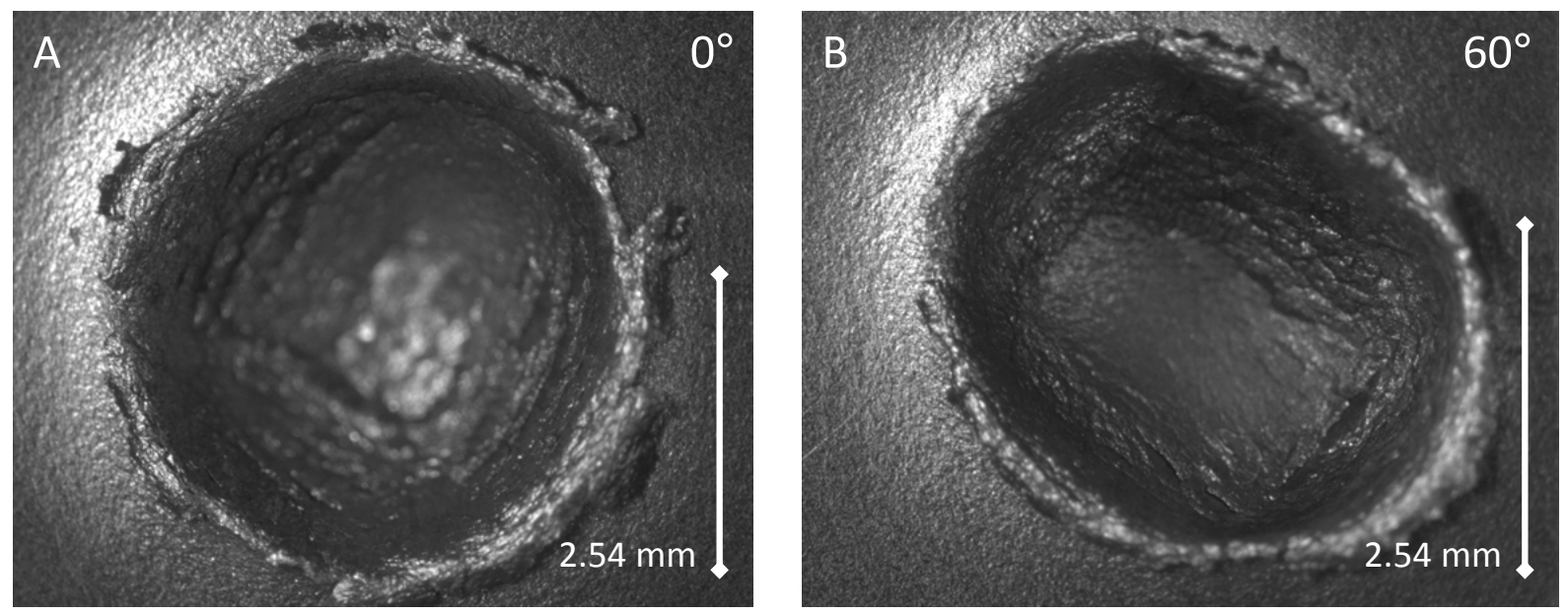

Figure 1.7.5: Plates of 1018 steel, $150 \mathrm{~mm}$ diameter and $10 \mathrm{~mm}$ thick, impacted at (A) $0^{\circ}$ obliquity and (B) $60^{\circ}$ obliquity by a right nylon cylindrical slug, $1.8 \mathrm{~mm}$ diameter and length, at $6.65 \mathrm{~km} / \mathrm{s}$. 


\subsection{Facility Efficiency}

One key difference of the gas-gun used in this investigation is that it has a much shorter flight tube, at approximately $4 \mathrm{~m}$, than the common 20 to $30 \mathrm{~m}$ long flight tubes found in many existing gas-gun facilities. Additionally, this gas-gun facility does not take any explosives or large amounts of gun powder to initiate impactor motion. As a result, the size of the launch package is smaller than most other facilities. Moreover, the test facility used in this investigation is a smooth bore facility, a consequence of the small impactor size, shorter length, and launch mechanism. While this design may attribute to the higher launch speeds attainable, it does so at a cost in accuracy and repeatability of the shot location. Presently, shot accuracy is within roughly 20 diameters of the impactor characteristic length.

Some of the inherent factors that contribute to the facility's overall efficiency include: the conversion of chemical energy in the gun powder to the kinetic energy of the piston and later the impactor, the friction of the piston and impactor in the pump and flight tubes, the alignment of the facility components, the vacuum seal on all interfaces, the amount of recoil on a shot, the slight variations in gun powder and wad amounts, the Mylar disc rupture or pre-rupture from blow-by effects, and the material and pressure wave speed coupling which affects the shock front and initial thrust timing, strength, and temperatures released onto the launch package, among others.

The design component of an AR section between stage 1 and stage 2 in the facility, although allowing for quick shock front acceleration, does so with some negative consequences on the facility. Specifically, high temperatures and pressures generated during a test can erode, and in some cases, plastically deform the steel components to the point where they have exceeded a safe limit and must be replaced. Consequently, a component of facility health monitoring must be performed in conjunction with testing. This is not a condition specific to this particular facility. In fact, Piekutowski and Poorman [40] have documented the facility degradation effect on AR-type acceleration light-gas guns, stating "Accelerated reservoir firing cycle guns exhibit launch-tube erosion [...] and at certain loading conditions produce deformation, erosion or other damage to launcher components". As a result, a new launch tube is used for every shot and safety limits are posed for AR section nozzle diameters, as well as the steel launch tube housing. 
Considering the gun powder burn or potassium nitrite combustion expression,

$$
10 \mathrm{KNO}_{3}+3 \mathrm{~S}+8 \mathrm{C} \rightarrow 2 \mathrm{~K}_{2} \mathrm{CO}_{3}+3 \mathrm{~K}_{2} \mathrm{SO}_{4}+6 \mathrm{CO}_{2}+5 \mathrm{~N}_{2}
$$

the energy of the reaction gets dissipated roughly between the following:

- Kinetic energy to bullet $29 \%$

- Kinetic energy to gases $19 \%$

- Heat to barrel $22 \%$

$\circ$ Heat to gases $19 \%$

- Heat to piston friction $7 \%$

- Heat to cartridge case $4 \%$.

Consequently, energy yield from the propellant burn of the facility is approximately $3.35 \mathrm{~kJ}$. As shown previously, the range of kinetic energies produced in the launch packages in this study are between 10 and $300 \mathrm{~J}$. Therefore, the facility has approximately $0.3 \%$ to $9 \%$ conversion of propellant energy to kinetic energy of the launch package. How the internal and terminal ballistics affect the resulting impact velocity are discussed in detail in later chapters.

\subsection{Outline}

The objective of this study is threefold. The first goal is to understand the internal and terminal ballistics of the unique two-stage light-gas gun facility as a means to decouple launch mechanism components and facility-induced behavior, from damage mechanisms purely due to target impact. Once known, the second objective is to optimize the facility for hypervelocity investigations using the principles of experimental statistics. By examining some of the post-mortem hypervelocity impact ballistic limit equations in the context of micrometeoroid and orbital debris risk mitigation, a slightly improved and more physically based plane stress monolithic ballistic limit equation is introduced, as well as the need to obtain quantitative information regarding damage evolution during the event itself. As a result, the most significant objective of the study is the in situ investigation of the dynamic fracture behavior of brittle polymers, with the goal of determining a valid fracture criterion which can be used to predict the reliability of the material being impacted under the extreme conditions of hypervelocity impact. 
The remainder of the thesis is outlined as follows:

- Chapter 2 describes two hypervelocimetry methodologies developed on the facility, examines velocity distributions, and discusses possible sources of uncertainty in velocity measurements.

- Chapter 3 presents a full-factorial design of experiments conducted on the facility to characterize the effects of specific factors on internal and external ballistic response, and to measure empirical error while maximizing impactor velocity.

- Chapter 4 examines terminal ballistic equations in context of micrometeoroids and orbital debris strikes, and presents a modification to the Charters expression.

- Chapter 5 investigates in situ dynamic fracture behavior of brittle polymers resulting from hypervelocity impact utilizing optical diagnostics.

- Appendix 1 provides additional experimental statistical analysis.

- Appendix 2 lists additional optics information and extensions on research.

- Supplemental 1 comprises of example hypervelocimetry test videos.

- Supplemental 2 presents high-speed photography results of dynamic fracture behavior. 


\section{Chapter 2}

\section{Hypervelocimetry}

One of the most fundamental challenges associated with hypervelocity impact empirical studies is developing robust and accurate velocimetry methodologies. This is particularly critical for the unique two-stage light-gas gun used in these investigations due to the fact that the projectiles are much smaller, averaging $1.8 \mathrm{~mm}$ in length and diameter, than comparable facilities. Consequently, more traditional means of measuring velocity where the projectile interrupts laser sheets or photodetectors, or perhaps breaks an electrical connection, are not practical given the speed and scale of the impactors. While the previously listed methods work well for larger projectiles or slower impacts, the two-stage light-gas gun in this study uses a projectile that is too small, traveling too quickly, to be accurately detected (or often detected at all) when using these methods alone. Additionally, highly energetic phenomena developed from the launch mechanism, as well as from the hypervelocity impact event, can create sources of interference and error in velocity measurement systems. In order to address these concerns, high-speed photography velocimetry methodologies were developed to capture a physical image history of the projectile flight and impact over a known time with minimal error and high reliability. 


\subsection{Detection and Error}

A Photron SA-1 Fastcam high-speed camera fitted with a $25 \mathrm{~mm}, 0.95 \mathrm{f}$ (optical aperture) objective lens was used to capture a video of the projectile in flight, as well as the impact event and post-impact phenomena. The camera was mounted on top of the target chamber, and focused down through an optical port in the plane of the incoming projectile at a known calibrated distance before the target. In addition, optical mirrors were mounted in such a way that the target could also be captured in the field of view of the high-speed camera, as shown in Figure 2.1.1. For all tests presented, the camera operated between 72000 and 200000 frames per second (taking an image approximately every 5 to $14 \mu \mathrm{s}$ ) with a field of view between roughly $75 \mathrm{~mm} \times 25 \mathrm{~mm}$ to $300 \mathrm{~mm} \times 100 \mathrm{~mm}$. Tantalum and nylon slugs were used to probe both a lower velocity regime $(<3 \mathrm{~km} / \mathrm{s})$ in the former case, and a higher velocity regime $(>3 \mathrm{~km} / \mathrm{s})$, in the latter case, as it is shown in a later chapter that launch package density dictates the impact speed.

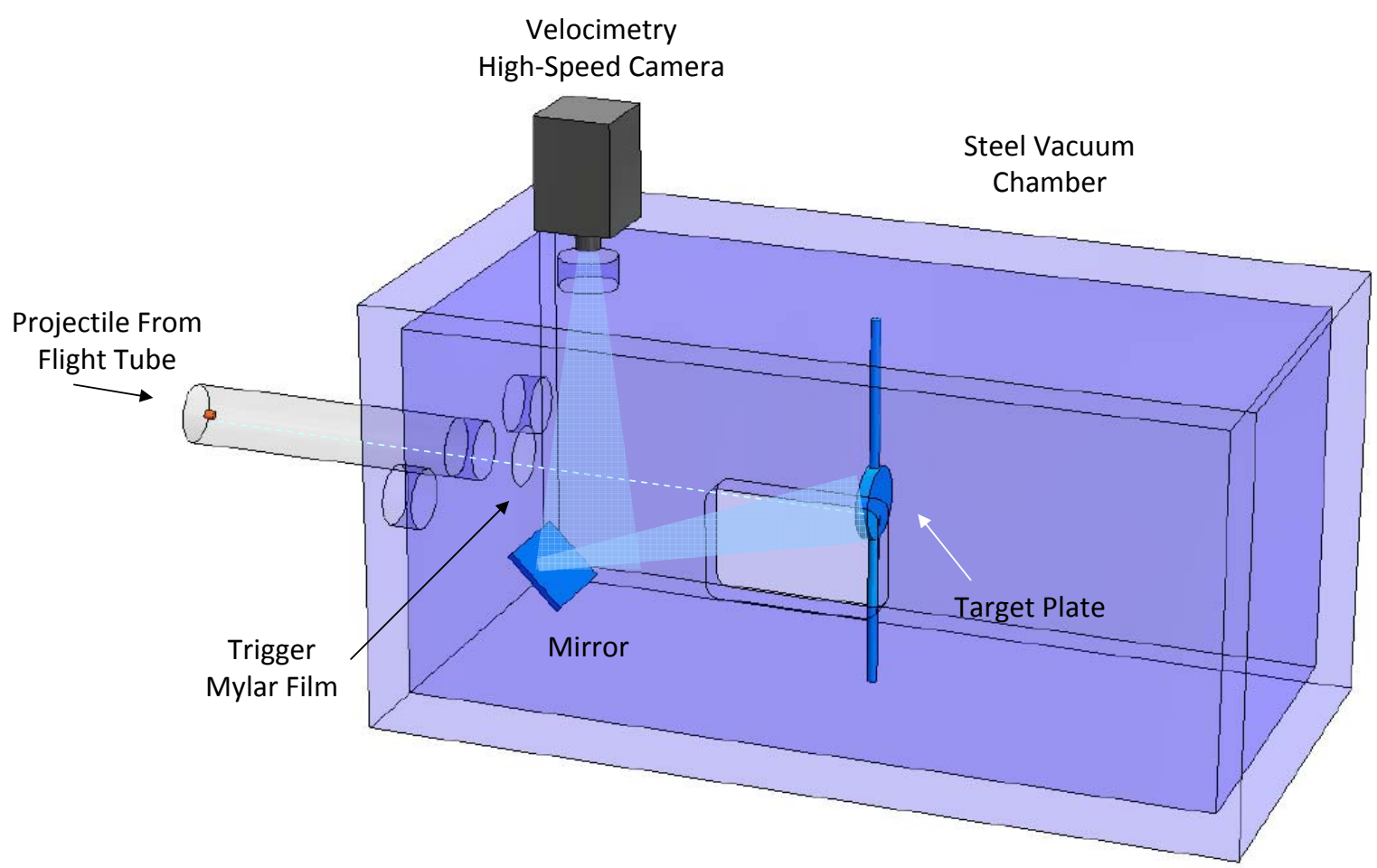

Figure 2.1.1: Velocimetry configuration for two-stage light-gas gun [16]

A trigger with a potentiometer was designed specifically for the facility ${ }^{1}$, to be used inside the target tank, and its circuit diagram is shown in Figure 2.1.2. The trigger has a variable resistor, $R_{1}$, which can be adjusted to a threshold value for a particular test configuration and location of

\footnotetext{
${ }^{1}$ Trigger designed and built by Tom Reynoso from NASA's Jet Propulsion Laboratory in 2007
} 
the diode. Through a symmetrical pair of p-type and n-type metal oxide semiconductor field effect transistor (MOSFET), the device has high noise immunity and low static power consumption. Upon excitation, a 5 V DC TTL (transistor to transistor logic) trigger is sent to the high-speed camera. Commercial high-speed silicon detectors with a wavelength range between 200 to $1100 \mathrm{~nm}, 0.8 \mathrm{~mm}^{2}$ active area, and a 1 ns response time, were used for an additional means of monitoring; but due to lack of reliability, were only configured in redundant velocity measurement systems.

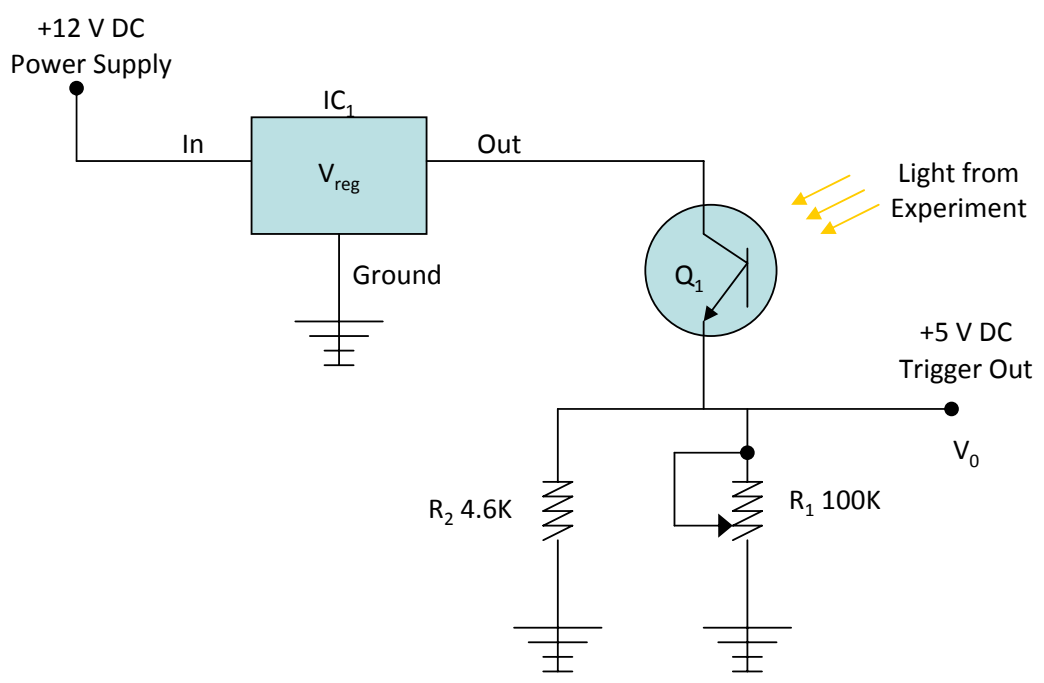

Figure 2.1.2: Trigger mechanism used for high-speed velocimetry in the gas-gun facility. $\mathrm{Q}_{1}$ sends a $5 \mathrm{~V}$ DC output upon excitation at a variable threshold region, $\mathrm{R}_{1}$

The gas-gun used in this investigation is approximately $6 \mathrm{~m}$ in total length, having a flight tube section of about $3 \mathrm{~m}$, or the portion of the gun where the impactor experiences free flight before entering the target chamber. Due to this relatively short flight tube, generating a clean separation distance between the impactor, muzzle flash structure ${ }^{2}$, and hot barrel gases can be problematic, and can lead to mis-triggering or interfering with velocity measurement systems. Additionally, highly energetic phenomena present at the impact site can also cause disturbances and triggering issues.

The facility has no source of muzzle brake or blast deflector between the launch tube and flight tube, and as a result muzzle flash and blast features are ejected downrange of the launch tube. There are five predominant components of muzzle flash, each with associated causes, wavelength emittance, and gas flow phenomenon. Pre-flash is the flash caused by blow-by, which is the condition where the light-gas leaks around the projectile or even earlier, around the Mylar disc in the AR section,

\footnotetext{
${ }^{2}$ In the case of the two-stage light-gas gun used in this investigation, muzzle refers to the downrange section of the launch tube where the projectile enters free flight.
} 
and exits the muzzle before the impactor. Blow-by is a consequence of inherent imperfections in the experimental configuration. Another consequence of imperfections in the launch system is that the projectile may not obturate perfectly from the muzzle, which could be due to gun wear, slight loading misalignment, or possibly friction.

A gun-powder launch also has a primary, intermediate, and secondary flash associated with the muzzle blast structure. Primary flash is the flash that occurs from solid powder particles or gases burning when the projectile exits the muzzle (not before the impactor, like pre-flash). The intermediate flash is the illumination that occurs when gas travels ahead of the normal shock at the muzzle, and is due to an increase in pressure and temperature of the light-gas as it passes through the shock front. The secondary flash is due to the reaction of the combustion products oxidizing when mixing with the low level of atmosphere present in the flight tube [4] as can be seen in Figure 2.1.3.

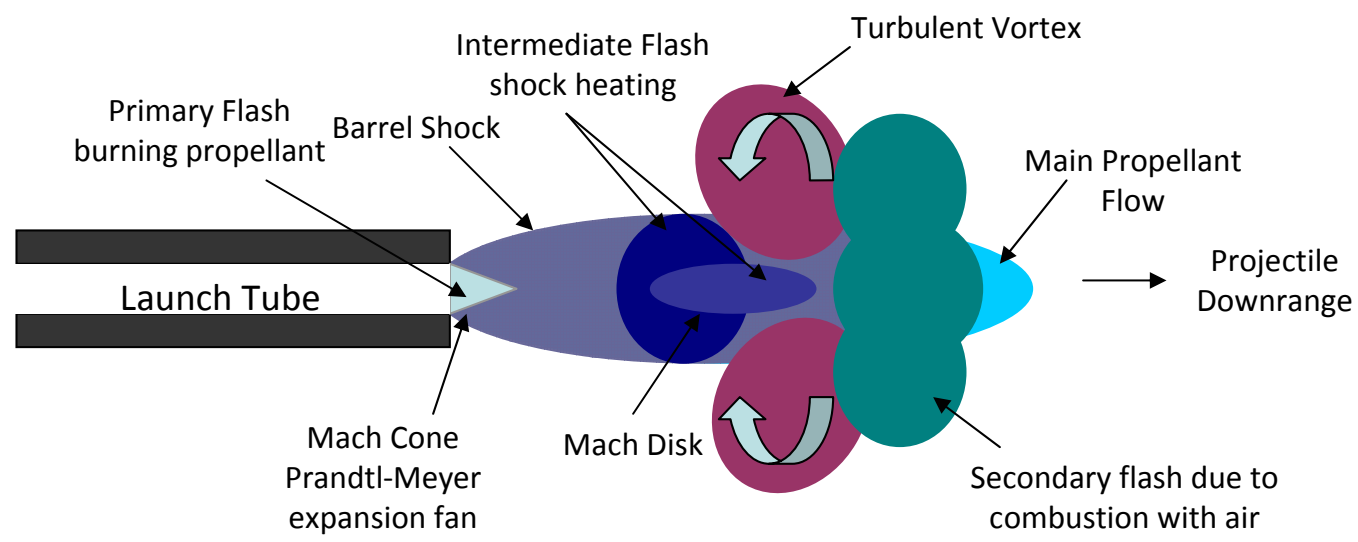

Figure 2.1.3: Muzzle blast structure on the downrange end of the launch tube which can affect velocity measurements [4]

In addition, muzzle glow occurs purely from the gas inside the shock bottle. The shock bottle is the shock and Mach cone that form after the pre-flash shock. The shock is created as the increasing pressure in the gas being compressed by the onrushing impactor attempt to push into the precursor flow field. In essence, the precursor flow field actually constrains the flow exiting the muzzle and a Mach cone is generated when the wall constraining the jet of gas exiting the muzzle disappears. As the flow reaches the end of the muzzle (launch tube) it looses the wall constraint, attempts to turn the corner, but cannot, so an expansion fan forms instead. As gases continue to jet out of the end of the muzzle, the gas near the outer boundaries looses momentum and forms a vortex. The vortex progresses downrange and in some cases can approach or even overtake the pre-flash [4]. 
At the point of impact in the target chamber, depending on the projectile and target materials as well as the speed of the collision, the spectral flash will have varying wavelength components. While it is possible to use only photodetectors at the muzzle flash and at the target flash to base a velocity measurement from, the accuracy or level of uncertainty in the velocity measurement can be large. This is because there are numerous sources of light at the muzzle due to the launch mechanism that are not present at the target, such as pre-flash which can cause pre-triggering, or and primary, intermediate, and secondary flashes which can cause post-triggering of the diode. All of these are not easy to decouple from the actual projectile release at the muzzle.

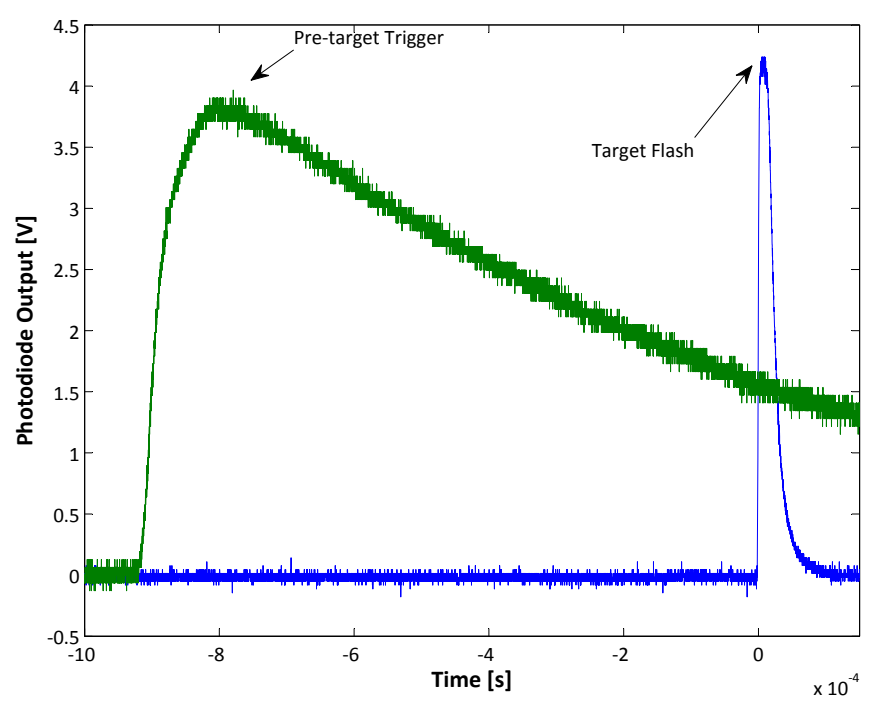

Figure 2.1.4: Photodiode trigger velocity system output from a hypervelocity impact test to illustrate the difference in diode response time and duration, which varies depending on the impactor and target materials, as well as the triggering event and location

In nearly all impact cases, ejecta will be thrown, often from both the front and rear surface of the plate, and can interfere with detectors, triggering, and diagnostics (shown in Figure 2.1.7). As a result of these possible impact events, rise time and detection wavelengths generated at the target will be different than those generated at the muzzle end, or at any location along the flight tube. An example showing the difference between the wavelength signals detected by photodiodes from a pre-target location and at target flash is shown in Figure 2.1.4. Moreover, impact velocities above approximately $3 \mathrm{~km} / \mathrm{s}$ generate a plasma sheath surrounding the moving projectile, and can pre-trigger or send electrical surges through the system. Videos of the high-speed photography velocimetry are shown in Supplemental 1. 

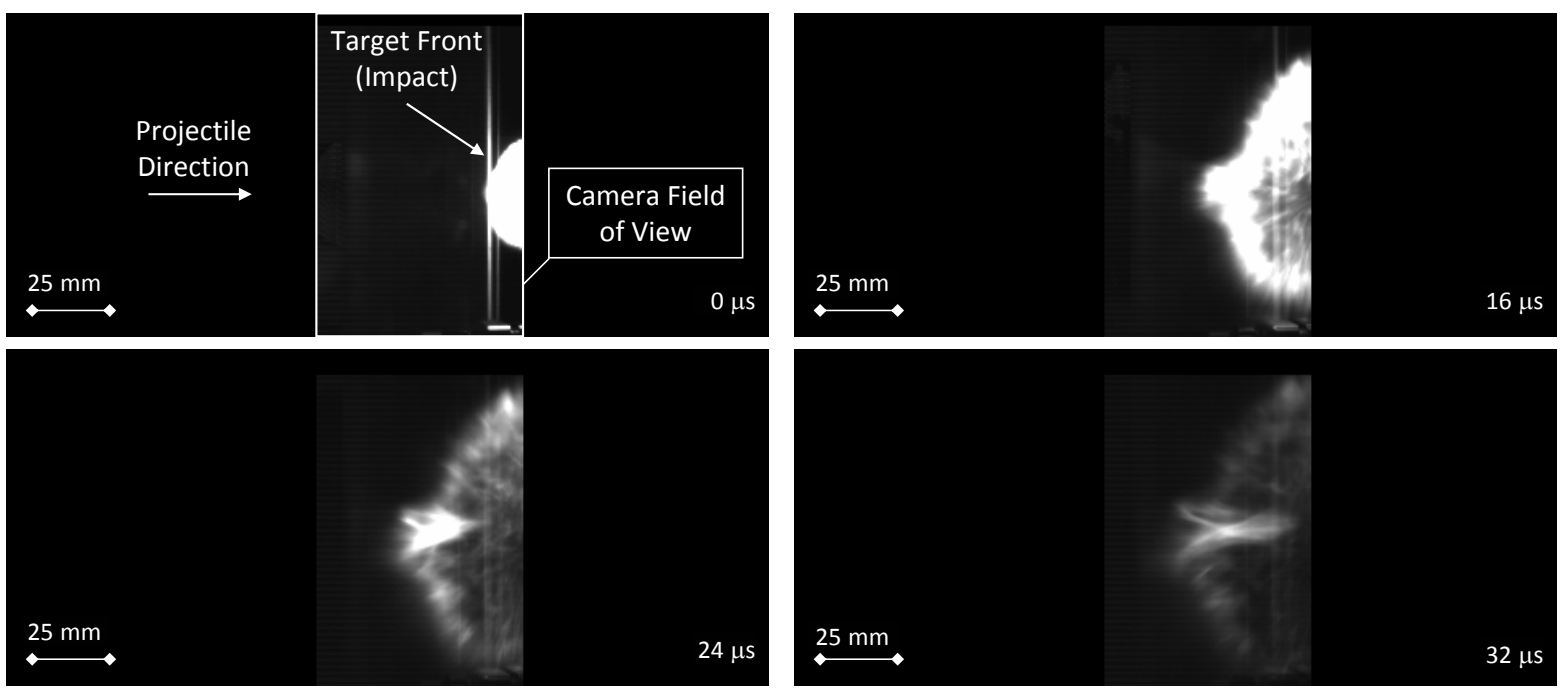

Figure 2.1.5: Example of forward ejecta captured by high-speed photography viewed from the side of a nylon cylindrical slug, $1.8 \mathrm{~mm}$ in length and diameter, penetrating a $150 \mathrm{~mm} \times 150 \mathrm{~mm} \times 12.7 \mathrm{~mm}$ Mylar plate at $4.5 \mathrm{~km} / \mathrm{s}$
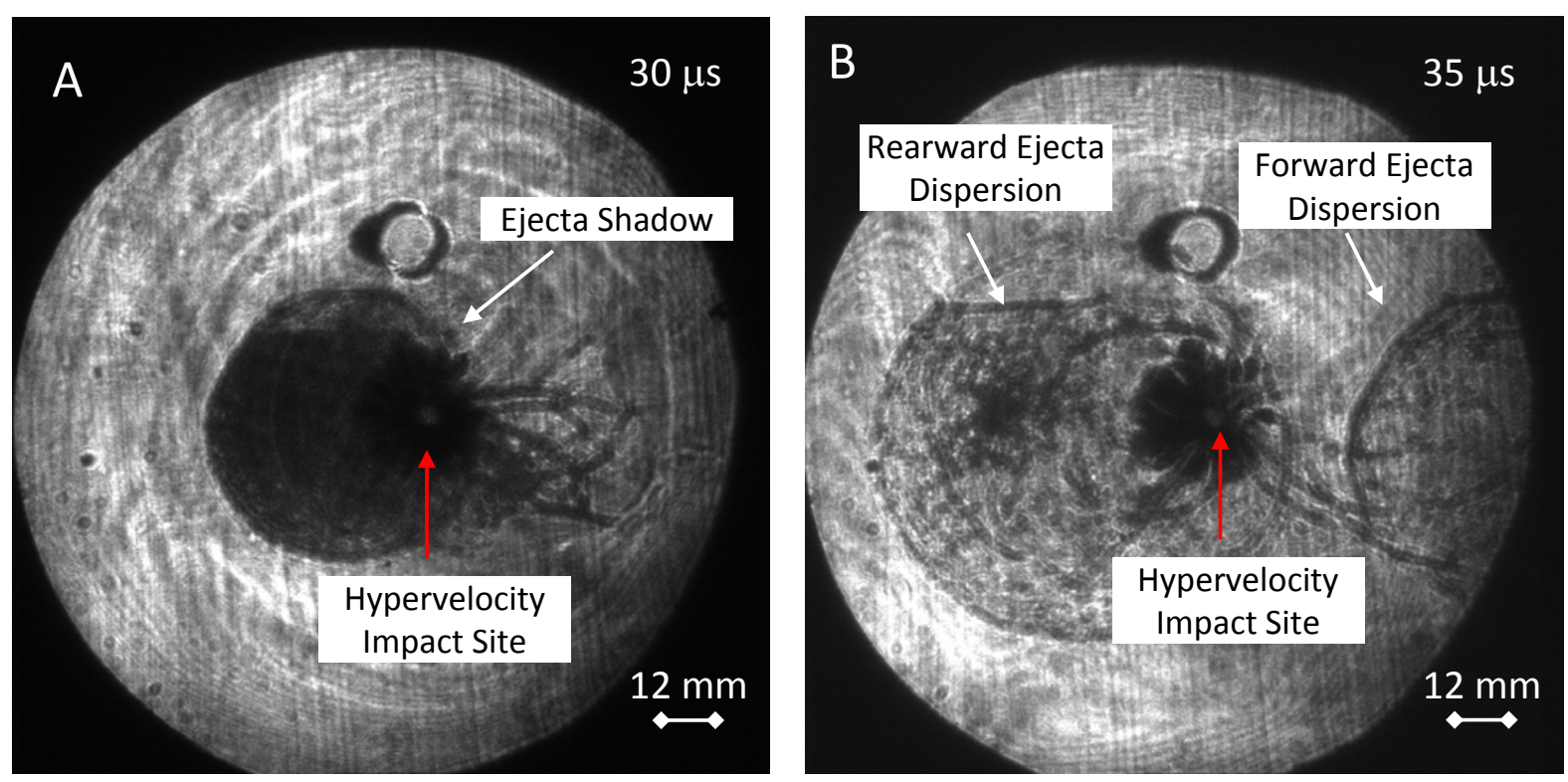

Figure 2.1.6: Shadowgraph of ejecta (A) $30 \mu \mathrm{s}$ after impact and (B) $35 \mu$ s after impact with nylon cylindrical slug, $1.8 \mathrm{~mm}$ in diameter and length, on Mylar plate, $150 \mathrm{~mm}$ diameter and $1.6 \mathrm{~mm}$ thick, at $6 \mathrm{~km} / \mathrm{s}$. Target has $20 \mathrm{~mm}$ hole and is loaded in $92 \mathrm{kPa}$ tension by Kevlar thread with weights 
In summary, muzzle blast structure, and in some cases the impactor plasma sheath, combined with target impact events such ejecta and plasma, may generate false triggers, impair velocity measurements, and add uncertainty in the true collision speed of the impactor. To address these issues, a few independent means of measuring hypervelocity were developed to minimize error, maximize reliability, and quantify sources of uncertainty.
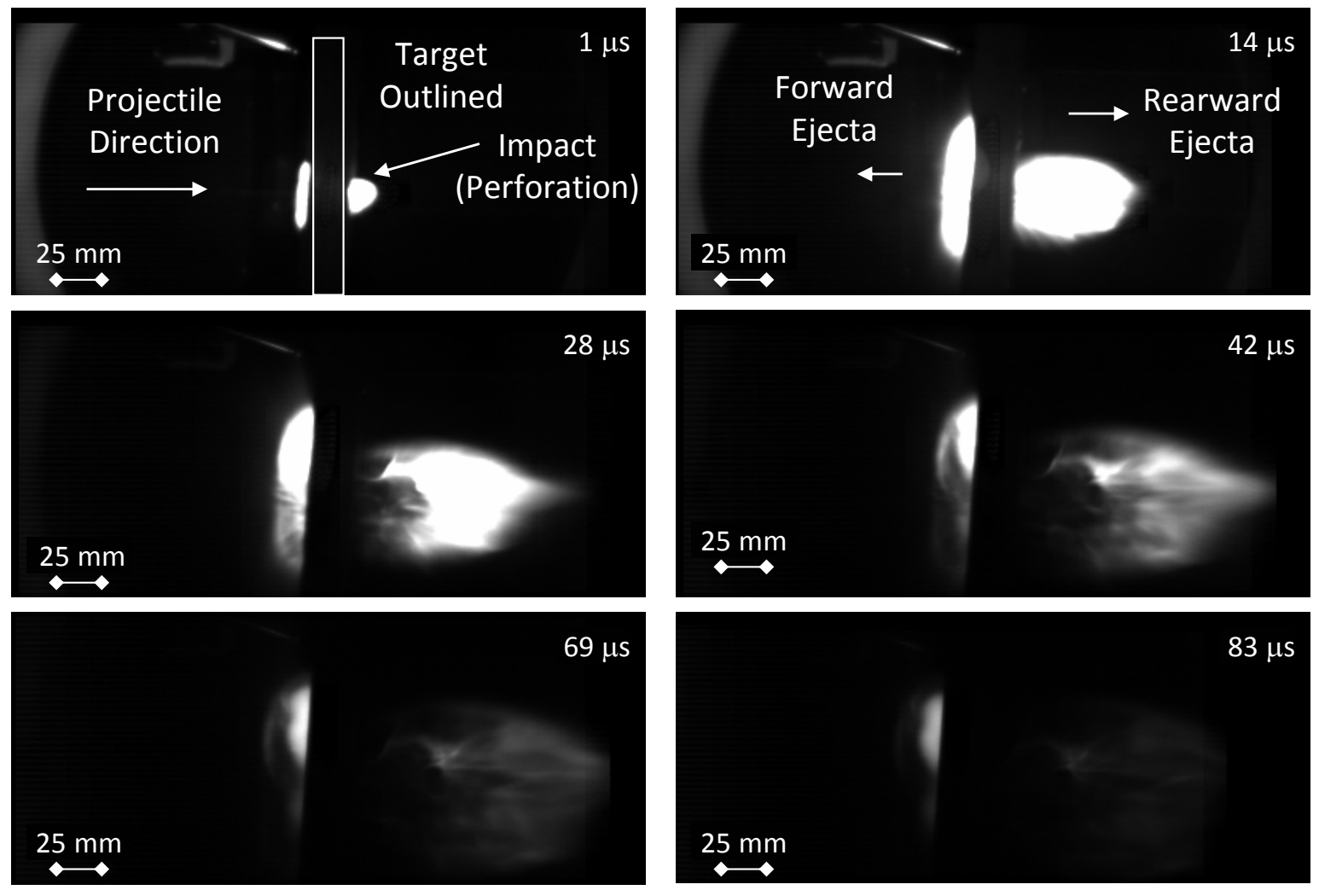

Figure 2.1.7: Example of forward and rearward ejecta, spectral flash, and plasma glow captured by high-speed photography viewed from the side of a nylon cylindrical slug, $1.8 \mathrm{~mm}$ in length and diameter, perforating, or creating a hole in, $150 \mathrm{~mm}$ diameter and $10 \mathrm{~mm}$ thick Homalite 100 plate at $4.9 \mathrm{~km} / \mathrm{s}$

\subsection{Method 1: Mylar Flash Method}

At the slowest region of hypervelocity examined in this study, namely collision speeds less than $3 \mathrm{~km} / \mathrm{s}$, no plasma sheath is generated around the impactor and consequently no visible light is produced by the impactor during flight. In order to determine the location of the impactor, a thin Mylar film $(12.7 \mu \mathrm{m})$ is placed at a known distance ahead of the target. The film is in the plane perpendicular to the velocity vector of the impactor, and in the field of view of the high-speed 
camera. When the impactor passes through the Mylar film, it produces a bright flash adequate for the camera to capture. Some frames later, a second flash is captured as the impactor hits the target, providing a means of estimating velocity. To attain velocities between less than $3 \mathrm{~km} / \mathrm{s}$, high density metallic impactors of tantalum and steel were used. Consequently, the pre-target Mylar film does little damage to the impactor. Uncertainty in the velocity measurement from this method comes from the camera field of view coupled with the framing rate. Each is the limiting factor for the other. This means that the larger field of view, the slower the available framing rates on the camera. The opposite is true as well, very fast framing rates must have small fields of view. Additionally, the pixel resolution versus impactor size adds uncertainty in the measurement, restricting the resolution of the length scale. Depending on the distance between the Mylar film and the target, as well as the speed of the projectile, uncertainty in the velocity measurement is on the order of $\pm 10 \%$ or less. An example from the Mylar flash is shown in Figure 2.2.1.
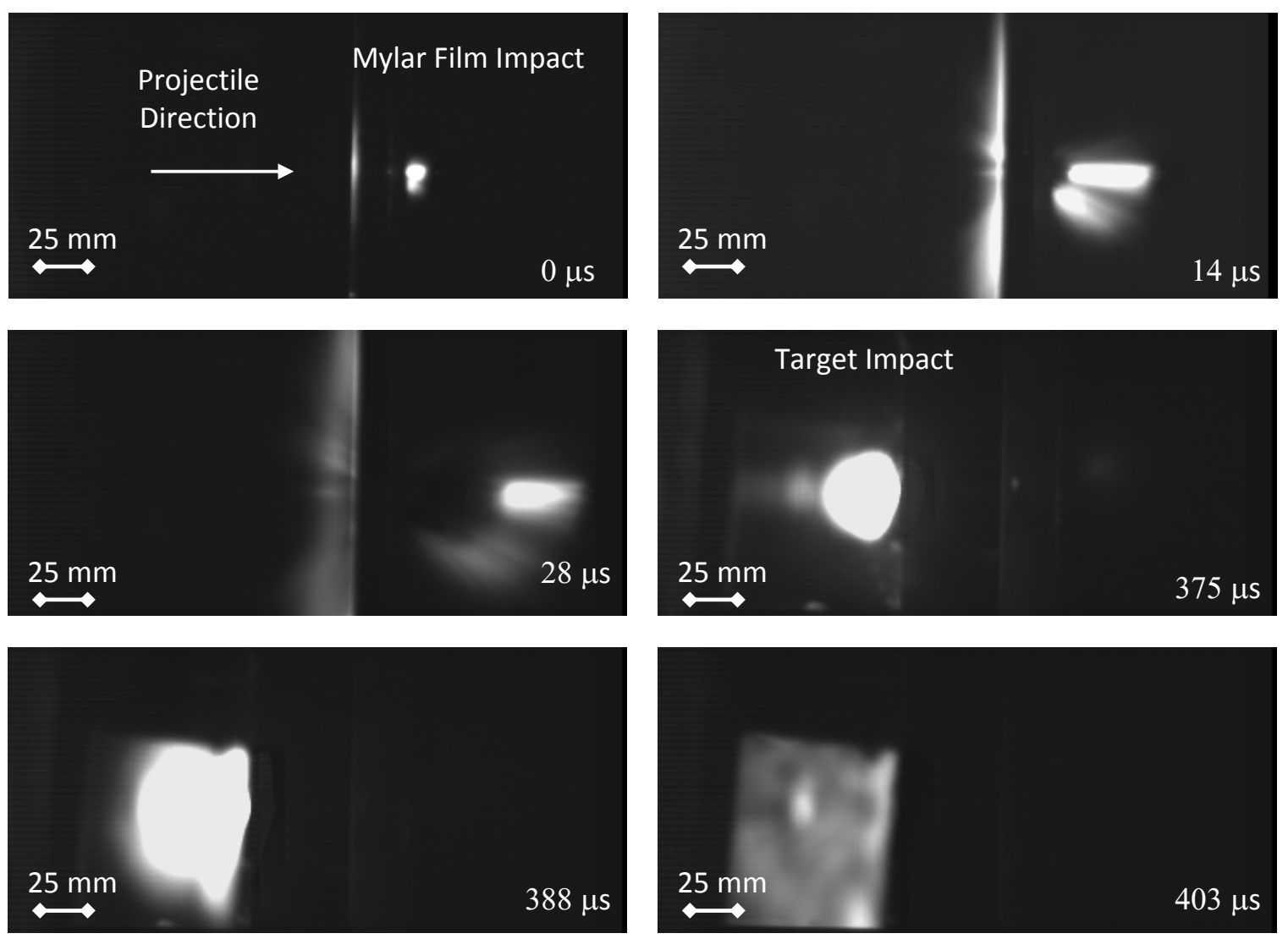

Figure 2.2.1: Mylar flash velocimetry method showing a 440C stainless steel sphere impactor, $1.8 \mathrm{~mm}$ in diameter, colliding with a 304 stainless steel plate, $150 \mathrm{~mm} \times 150 \mathrm{~mm} \times 2.7 \mathrm{~mm}$ at $0^{\circ}$ obliquity and $2.67 \mathrm{~km} / \mathrm{s} \pm 6.5 \%$. The Mylar film impact is directly in the camera field of view, whereas the target plate is shown in the reflection of an optical mirror and is located $0.64 \mathrm{~m}$ downrange 


\subsection{Method 2: Streak Method}

When the impactor is traveling at speeds greater than $3 \mathrm{~km} / \mathrm{s}$, the kinetic energy is high enough such that, when combined with the low-pressure present in the flight tube and target chamber $(0.13$ to $2.4 \mathrm{kPa}$ ), gas particles are ionized around the moving projectile and create a self-illuminating streak or plasma sheath. In this case, the impactor is bright enough to be seen by the high-speed camera and can be used, when examined frame-by-frame, to generate an accurate time-distance history. Although a shock is present ahead of the impactor, it remains the same relative distance from the impactor during flight. As a result, the head of the shock front is used to determine the impactors location without adding additional uncertainty in the velocity measurement. Like the Mylar flash method, the high-speed camera framing rate, coupled with the field of view, dictate the uncertainty. The streak method typically has an uncertainty on the order of $\pm 2 \%$ or less, depending on the configuration.
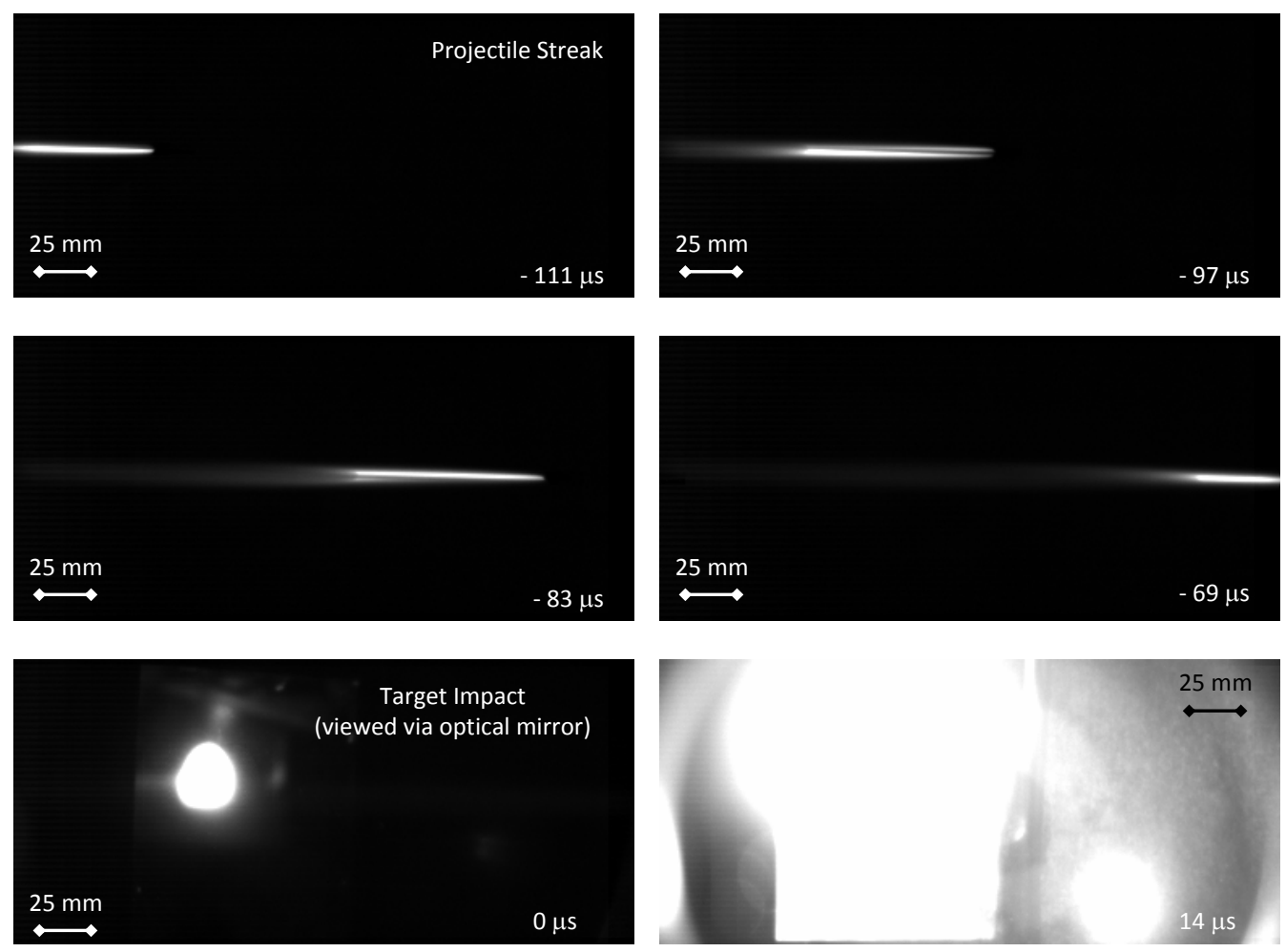

Figure 2.3.1: Streak velocimetry method showing plasma sheath around nylon cylindrical impactor, $1.8 \mathrm{~mm}$ in diameter and length, at a series of instances in time, colliding with an Aluminum 6061-T6 plate, $150 \mathrm{~mm} \times 150 \mathrm{~mm} \times 25 \mathrm{~mm}$ at $60^{\circ}$ degrees obliquity at $6.5 \mathrm{~km} / \mathrm{s} \pm 2 \%$. The streak of the impactor with a plasma sheath is directly in the camera field of view, whereas the target plate is shown in the reflection of an optical mirror and is located $0.64 \mathrm{~m}$ downrange 


\subsection{Velocimetry Comparison}

In the lower velocity regime tested $(<3 \mathrm{~km} / \mathrm{s})$, the streak method could be utilized in a slightly different manner since the impactor gives off light a few frames after impact with the pre-target Mylar film. This method is referred to as the hybrid streak method. In the higher velocity regime tests $(>3 \mathrm{~km} / \mathrm{s})$ when nylon cylindrical slugs were used as the impactor, both the Mylar flash and streak velocimetry methodologies can be used. A graph of how the methods compare for each velocity regime is shown in Figures 2.4.1 and 2.4.2.

Overall, neither method showed immense deviation from the other, and both methods had statistically the same standard deviation. This observation was validated by performing an F-test and the details are shown in Appendix A. Movies from these two hypervelocimetry methodologies can be viewed in Supplemental 1.

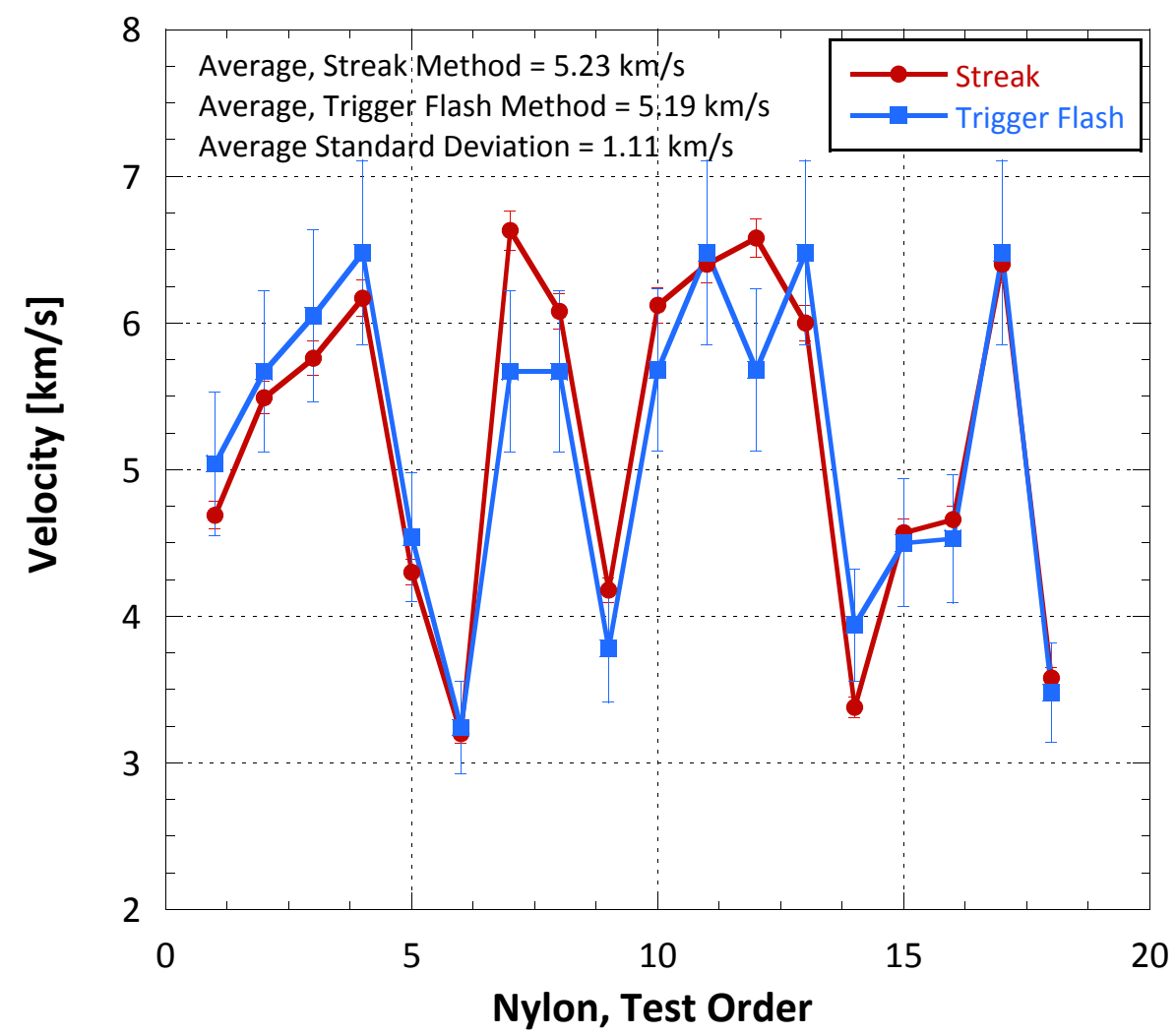

Figure 2.4.1: Graph of hypervelocity impact tests using nylon cylindrical slugs, $1.8 \mathrm{~mm}$ length and diameter, with velocity readings for both the Mylar flash and streak methods 


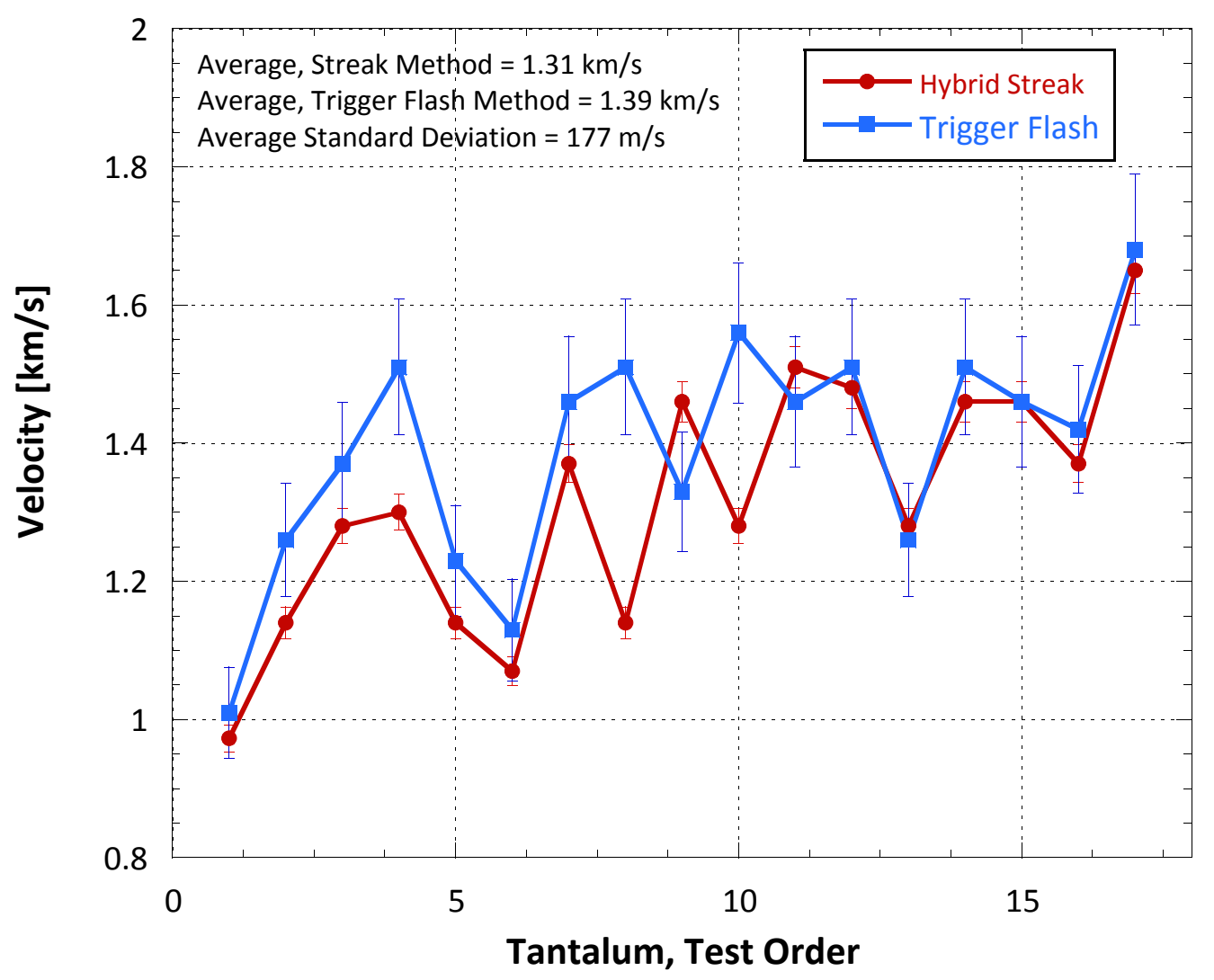

Figure 2.4.2: Graph of hypervelocity impact tests using tantalum sphere, $1.8 \mathrm{~mm}$ diameter, with velocity readings for both the Mylar flash and streak methods 


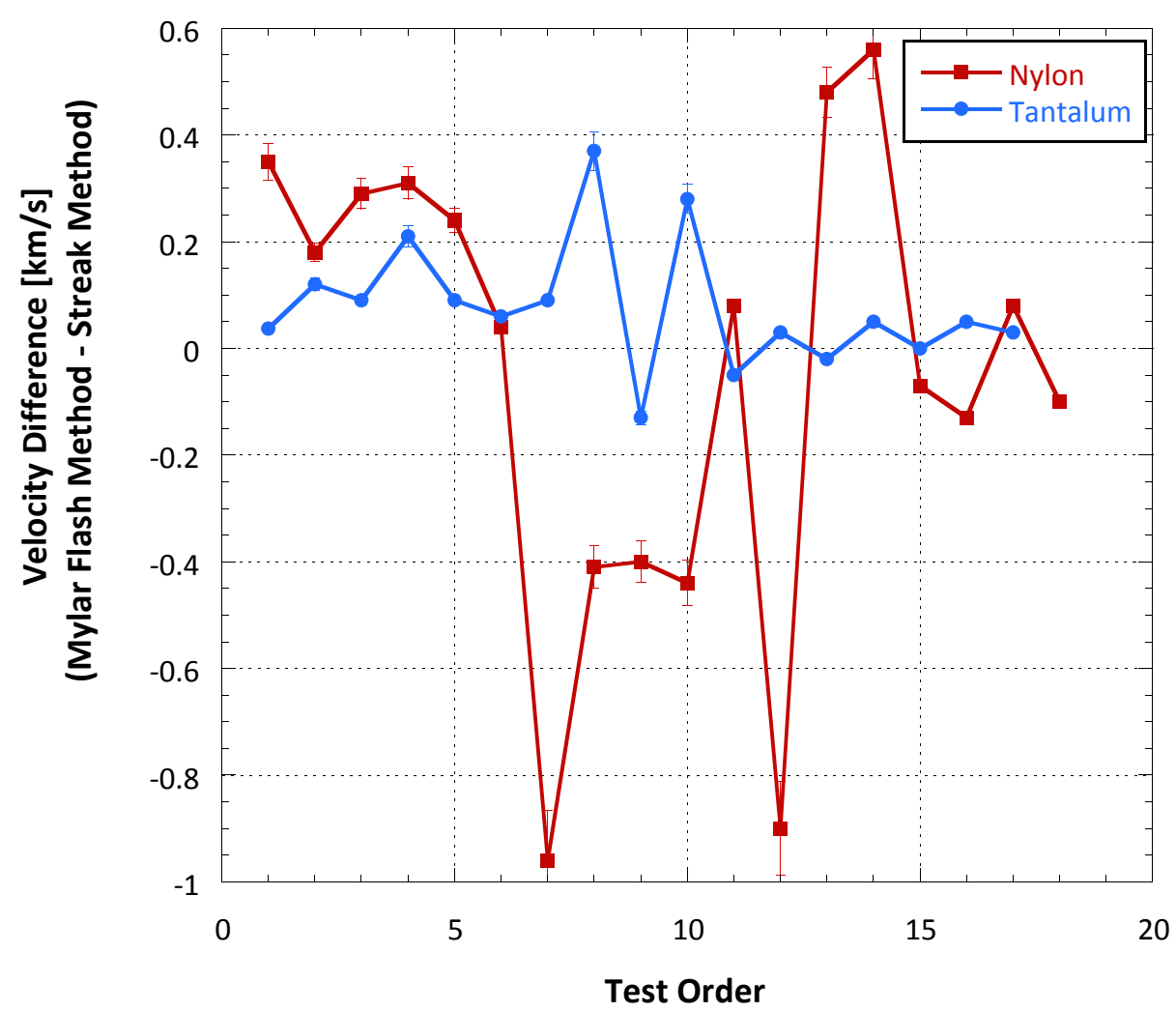

Figure 2.4.3: Graph of the difference between the Mylar flash method and the streak method of measuring hypervelocity. Both sets of experiments from nylon right cylindrical slugs at speeds greater than $3 \mathrm{~km} / \mathrm{s}$, and tantalum spheres at speeds less than $3 \mathrm{~km} / \mathrm{s}$, are shown

These high-speed-photography-based methods of velocimetry may be configured simultaneously for redundancy, and uncertainty in the measurement is dictated by the coupled effect of the framing rate with the camera field of the view. It is important to note that by using these optical methodologies to determine velocity, the high-speed camera frames provide a two-dimensional representation of a three-dimensional event. Therefore, small amount of error unaccounted for in the third dimension may exist. If the impactor has some pitch associated with the flight path, it would slightly skew the calculated velocity from the Mylar flash or streak method. However, since the change in length in the direction of flight is much greater than the length that may be associated with the small variation in out-of-plane location during free flight, this error is considered negligible.

Due to the atmosphere present in the target tank (minimum $0.13 \mathrm{kPa}$ ) in the higher velocity regime case with nylon slugs, a shock develops ahead of the moving impactor as shown in the shadowgraph in Figure 2.4.4. However, the conventional bow shock angle-inverse Mach relation should not be 
used to determine the impactor velocity. Assuming standard conditions, the oblique shock relation between the shock angle, $\beta$ and the Mach number of impactor, $M$, is

$$
1 / M=\sin \beta .
$$

To determine the uncertainty of the expression, the derivative of 2.4 .1 is

$$
d \beta / d M=\frac{-M^{3}}{\sqrt{M^{2}-1}},
$$

and reveals that a small change in $\beta$ corresponds to a large change in Mach number, on the order of $M^{2}$. Conversely, when $M$ is large (like in hypervelocity), the corresponding change in shock angle, $\beta$, will tend towards approximately $-1 / M^{2}$, or quite insignificant relative to the Mach. This simple differentiation illustrates the insensitivity of a bow shock angle velocity method at high Mach numbers.

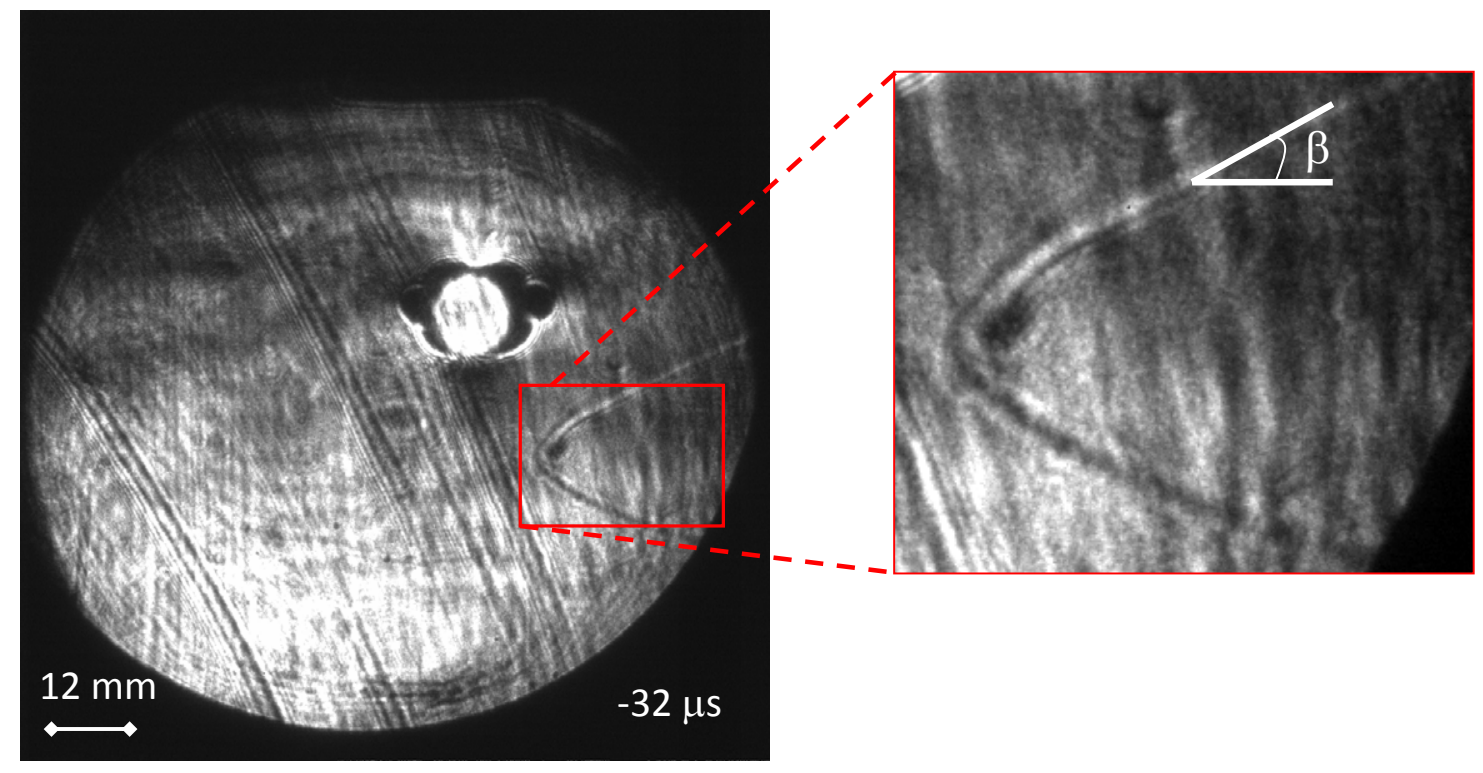

Figure 2.4.4: High-speed photography shadowgraph of a bow shock ahead of a moving nylon cylindrical impactor, $1.8 \mathrm{~mm}$ in diameter, traveling at $4.23 \mathrm{~km} / \mathrm{s}$ in $2.1 \mathrm{kPa}$ (16 Torr) atmosphere inside the target chamber 


\subsection{Velocity Distribution}

In both the lower velocity regime $(1-3 \mathrm{~km} / \mathrm{s})$ explored with tantalum spheres and the higher velocity regime $(>3 \mathrm{~km} / \mathrm{s})$ explored with nylon cylindrical slugs, the velocity distribution seems to be adequately defined by a normal distribution, shown in Figures 2.5.1 and 2.5.2. While other distributions may equally be argued as adequate distributions, an Anderson-Darling test was used to test for normality, and details are found in Appendix A.

The normal probability plots were generated with MINITAB ${ }^{3}$ and are shown in Figures 2.5.1 and 2.5.2. Both the tantalum impactors at lower velocities, as well as the nylon impactors at higher velocities, have P-values (listed on the right of the plots) greater than 0.05 and can be assumed to not deviate from normality.

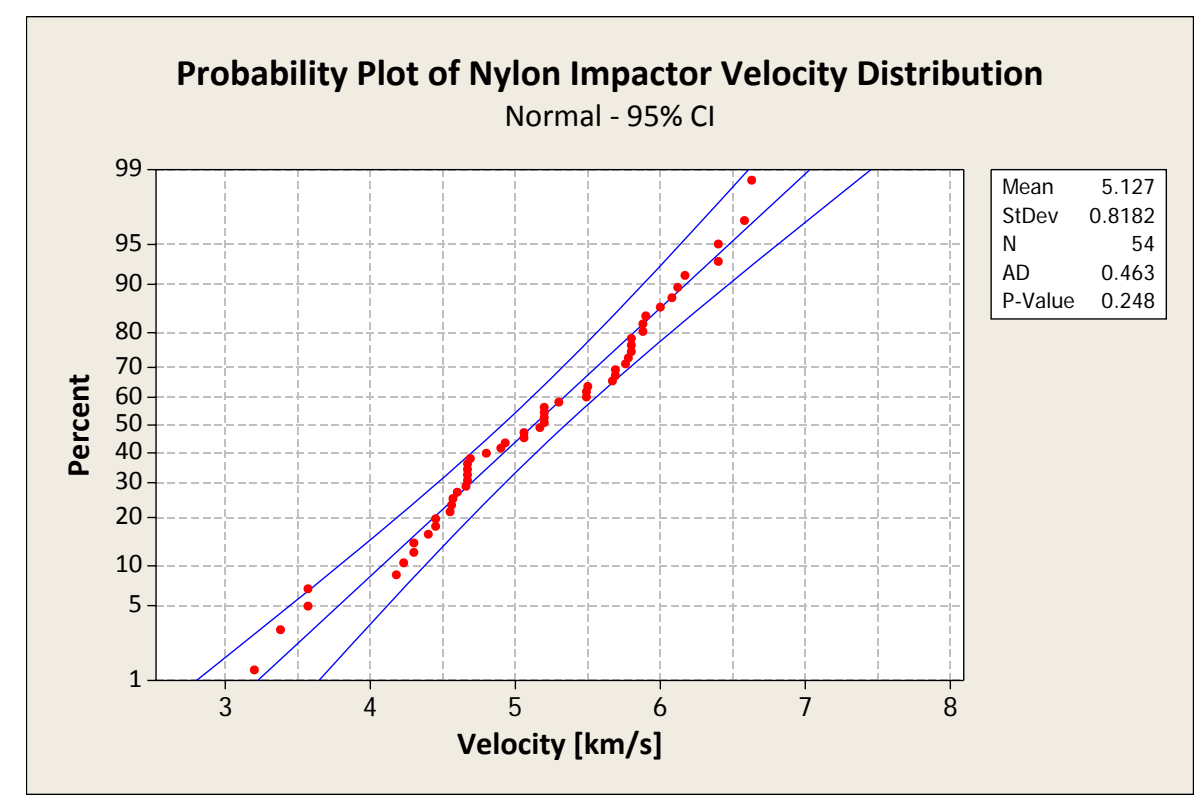

Figure 2.5.1: Normal distribution probability plot of velocity for nylon cylindrical projectiles, $1.8 \mathrm{~mm}$ in diameter and length, with a $95 \%$ confidence interval

\footnotetext{
${ }^{3}$ MINITAB, Release 15, 2007, www.minitab.com
} 


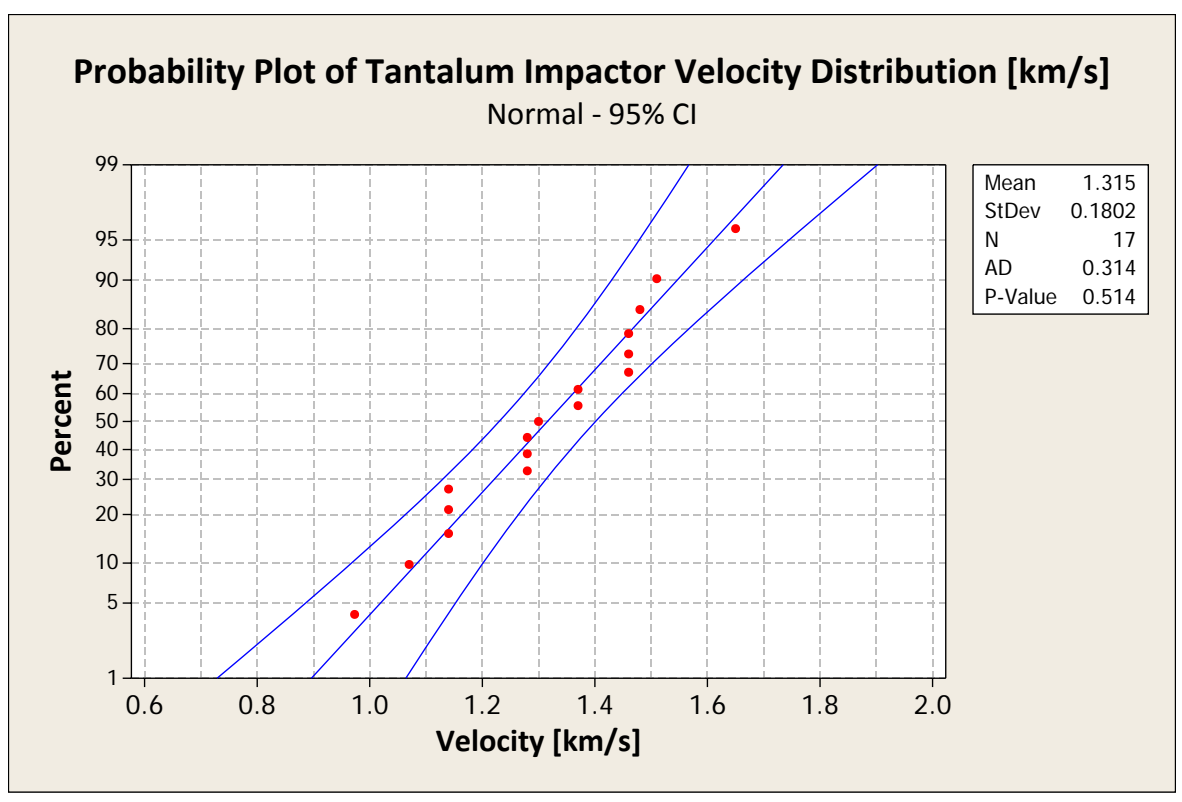

Figure 2.5.2: Normal distribution probability plot of velocity for tantalum sphere projectiles, $1.8 \mathrm{~mm}$ in diameter, with a $95 \%$ confidence interval 


\section{Chapter 3}

\section{Statistical Analysis of Hypervelocimetry}

Development of well-controlled and replicable hypervelocity launch capabilities is a crucial step to improve the understanding of material behavior in these complex impact regimes. Due to the fact that the two-stage light-gas gun facility used to carry out the experiments is relatively unprecedented in design and scale, and never previously performed a controlled set of experiments examining variational affects, the internal and external ballistic factors and their overall effect on the resulting velocity and consequent impact damage remained unknown. To address this issue, a design of experiments was carried out on the facility.

\subsection{Design of Experiments}

A full factorial design of experiment was chosen due its advantages over other classical design of experiments (DOE) including ease of implementation, straightforward analysis, and most importantly ability to ascertain factor interactions which improves with each factor considered. Determining interactions between factors is not possible with numerous DOE such as a Latin square design ${ }^{1}$, and using multiple t-tests runs a higher risk of committing Type $I$ error. ${ }^{2}$ Additionally, a full factorial approach is more efficient than studying one factor at a time. In this case a split-plot design was not necessary since a completely randomized run order was achievable, as well as replications. It should be noted that the main disadvantage with a full factorial design is that the test matrix size grows multiplicatively with each factor considered since all possible treatment combinations must be run; yet no database of systematic shots had ever been generated on this hypervelocity impact facility,

\footnotetext{
${ }^{1} \mathrm{~A}$ Latin square design can handle three factors each at the same number of levels and requires fewer runs than a full factorial, but interactions cannot be present.

${ }^{2}$ Type $I$ error occurs when the null hypothesis that is really true is rejected.
} 
making a full-factorial DOE a more favorable choice [29].

\subsection{Analysis of Variance Model}

The goal of a full-factorial design is to conduct true experiments to characterize response and empirical error, in order to provide the combination of independent variables which produces a maximum response of the yield variable [21]. In this study the yield variable, $y$, was velocity. In other words, the goal of the DOE was to determine the controllable factors on the two-stage light-gas gun which could have a statistically significant effect on the resulting impact velocity. Consequently, the null hypothesis of the DOE is "no treatment effect", meaning that the factor or factor combination has no effect on velocity.

Three controllable internal and terminal ballistic factors were considered. These included: hydrogen pressure in the pump tube which makes up the first stage of the gas-gun uprange of where the launch package is loaded, vacuum level (the amount of atmosphere present in the flight tube and target chamber that the projectile flies through), and launch package density (a function of projectile mass and geometry). Based on the criteria provided by Southwest Research Institute, the original designer of the gas-gun facility, the envelope of safe operation included approximately 0.7 to $2 \mathrm{MPa}$ (100 to $200 \mathrm{psi}$ ) of hydrogen gas pressure in the pump tube, and approximately 0.14 to under $3 \mathrm{kPa}$ (1-20 Torr) vacuum in the flight tube and target chamber. Consequently three levels of hydrogen gas, 0.7, 1, and 1.4 MPa (100, 150, and $200 \mathrm{psi})$, and three levels of vacuum, 0.14, 1, and $2 \mathrm{kPa}(1$, 7.5 and 15 Torr) were considered in the DOE. In addition, the most dense and least dense slug-type launch packages were used to vary launch package geometry and mass. These included tantalum spheres $1.8 \mathrm{~mm}$ in diameter with a density of $16.65 \mathrm{~g} / \mathrm{cm}^{3}$, and nylon right-cylindrical slugs $1.8 \mathrm{~mm}$ in length and diameter with a density of $1.14 \mathrm{~g} / \mathrm{cm}^{3}$. All three factors in the experiment are fixed, so a simple linear model can be adopted to test the hypotheses about the main effects and interactions.

In fundamental form, the two-stage light-gas gun full factorial model is given by

$$
y_{i j k m}=\mu+\tau_{i j k}+\epsilon_{m(i j k)}
$$

where $y_{i j k m}$ is the observed velocity response, $\mu$ is the common effect for the entire set of experiments, $i=1,2,3$ corresponding to the three levels of hydrogen pressure, $j=1,2,3$ corresponding to the three levels of vacuum, $k=1,2$ for the two levels of launch package density, $m=1,2$ for the

2 observations (replications) of each $i, j, k$ treatment combination, $\tau_{i j k}$ is the eighteen treatment 
combinations, and $\epsilon_{m(i, j, k)}$ is the error within each of the treatments. To complete the DOE, a total of 36 experiments in random order were conducted with these factors and the resulting velocity observation recorded. The results of the tests are presented in Table 3.1.

If you further split up $\tau$ into its three factors: hydrogen pressure, $A_{i}$, vacuum level, $B_{j}$, and launch package density, $C_{k}$, equation (3.2.1) can be expanded to show possible two- and three-way interaction between variables as

$$
\begin{aligned}
y_{i j k m}=\mu+A_{i}+B_{j}+C_{k}+(A B)_{i j} & (A C)_{i k}+(B C)_{j k}+(A B C)_{i j k}+\epsilon_{m(i j k)} \\
i & =1,2, \ldots, a, \\
j & =1,2, \ldots, b, \\
k & =1,2, \ldots, c, \\
m & =1,2, \ldots, n .
\end{aligned}
$$

Each of the three main effects has a degree of freedom equal to the number of levels of the factor minus one, and the degree of freedom for an interaction is the product of the the degree of freedom associated with the individual components of the interaction. In this model $a$ is equal to 3 for the number of levels of hydrogen pressure, $b$ is equal to 3 for the number of levels of vacuum considered, $c$ is equal to 2 for the number of launch package densities considered, and $m$ is 2 for the number of replications of each observation.

Analysis of variance models (ANOVA) rely on the sum of squares (SS) approach. The sum of squares partitioning for a three-way analysis of this type is shown in Figure 3.2.1.

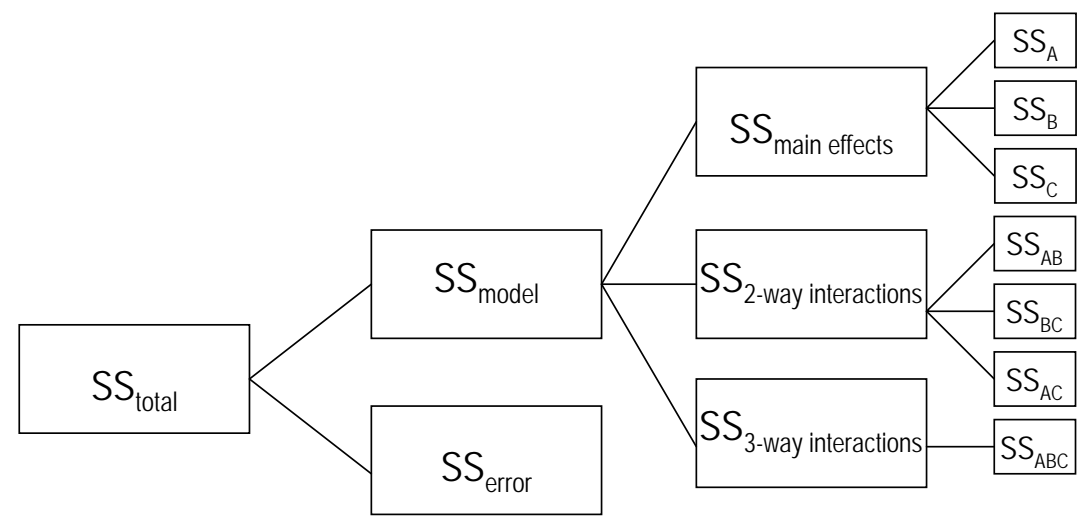

Figure 3.2.1: Sum of squares partitioning for three-way analysis of variance model 
Table 3.1: Hypervelocity observations from DOE tests varying launch package, hydrogen pressure, and vacuum levels on two-stage light-gas gun

\begin{tabular}{|c|c|c|c|c|}
\hline $\begin{array}{l}\text { Run } \\
\text { Order }\end{array}$ & Launch Package & Hydrogen Level [MPa] & Vacuum Level $[\mathrm{kPa}]$ & Velocity $^{\dagger}[\mathrm{km} / \mathrm{s}]$ \\
\hline 1 & Nylon & 1 & 0.14 & 4.69 \\
\hline 2 & Tantalum & 1.4 & 0.14 & 0.973 \\
\hline 3 & Nylon & 1.4 & 1 & 5.49 \\
\hline 4 & Tantalum & 0.7 & 0.14 & 1.14 \\
\hline 5 & Nylon & 1.4 & 0.14 & 5.76 \\
\hline 6 & Nylon & 1.4 & 2 & 6.17 \\
\hline 7 & Tantalum & 1 & 2 & 0.932 \\
\hline 8 & Tantalum & 1.4 & 1 & 1.28 \\
\hline 9 & Tantalum & 0.7 & 2 & 1.30 \\
\hline 10 & Tantalum & 1 & 1 & 1.14 \\
\hline 11 & Nylon & 1 & 2 & 4.30 \\
\hline 12 & Nylon & 0.7 & 2 & 3.20 \\
\hline 13 & Tantalum & 1 & 0.14 & 1.07 \\
\hline 14 & Nylon & 0.7 & 0.14 & 6.63 \\
\hline 15 & Nylon & 0.7 & 1 & 6.08 \\
\hline 16 & Tantalum & 1.4 & 2 & 1.37 \\
\hline 17 & Tantalum & 0.7 & 1 & 1.14 \\
\hline 18 & Nylon & 1 & 1 & 4.18 \\
\hline 19 & Nylon & 0.7 & 1 & 6.12 \\
\hline 20 & Nylon & 0.7 & 2 & 6.40 \\
\hline 21 & Nylon & 1.4 & 0.14 & 6.58 \\
\hline 22 & Tantalum & 1 & 1 & 1.46 \\
\hline 23 & Tantalum & 1 & 2 & 1.28 \\
\hline 24 & Tantalum & 0.7 & 0.14 & 1.51 \\
\hline 25 & Tantalum & 1.4 & 1 & 1.48 \\
\hline 26 & Nylon & 1 & 2 & 6.00 \\
\hline 27 & Nylon & 0.7 & 0.14 & 3.38 \\
\hline 28 & Tantalum & 1.4 & 2 & 1.28 \\
\hline 29 & Tantalum & 0.7 & 1 & 1.46 \\
\hline 30 & Nylon & 1.4 & 1 & 4.57 \\
\hline 31 & Tantalum & 0.7 & 2 & 1.46 \\
\hline 32 & Tantalum & 1 & 0.14 & 1.37 \\
\hline 33 & Tantalum & 1.4 & 0.14 & 1.65 \\
\hline 34 & Nylon & 1 & 1 & 4.66 \\
\hline 35 & Nylon & 1 & 0.14 & 6.40 \\
\hline 36 & Nylon & 1.4 & 2 & 3.57 \\
\hline
\end{tabular}


This model assumes that the error terms, $\epsilon_{i j k}$, are normally and independently distributed with mean zero and variance $\sigma^{2}$. Variance in this discrete case is defined as

$$
\sigma^{2}=\frac{\sum(y-\mu)^{2}}{N}
$$

where $\mu$ is the mean and $N$ is the total number of observations. The model also assumes that the observations are normally and independently distributed. Consequently, the total sum of squares $\left(S S_{T}\right)$ is a sum of squares of normally distributed variables. Each sum of squares divided by its corresponding degrees of freedom is a mean square (MS). In the case of fixed effects models, test statistics for each main effect and interaction may be constructed by dividing the corresponding mean square for the effect or interaction by the error mean square [21]. The error mean square is simply the average variance observed under the same conditions and is true when you have the same number of observations per cell. It holds true in this model since each treatment combination is replicated, so there are two observations per cell. This implies that if the null hypothesis were true and all the factors and possible combinations of factors made no difference on the resulting velocity, then all mean squares, $M S_{A}, M S_{B}, M S_{A B}, M S_{C}$, and so on, would all estimate $\sigma^{2}$. Conversely if there are levels of a factor that do make a difference in velocity, then the corresponding mean square would be larger than the error mean square, $M S_{E}$. Table 3.2 illustrates the variance partitioning for the three-factor fixed effects gas-gun facility model. Note that each row in the ANOVA table represents a null hypothesis in which the means of each factor level are equal.

Using this hypothesis testing statistical technique, it is assumed that the sum of independent and normally distributed variables is approximately normally distributed, as justified by the Central Limit Theorem [36]. Thereby a chi-square sampling distribution can be assumed for each of the variables in the model, and the ratio of mean squares are assumed $F$ distributed. All ANOVA models calculate an $F$ statistic $\left(F_{0}\right)$ that is assumed to be $F$ distributed, and $P$ value for each row. The critical output of the DOE is therefore the value of the response in the upper tail of the $F$ distribution which corresponds to the $F$ statistic used in determining the $P$ value. Integrating the $F$ distribution from zero to the ratio of mean squares in a particular case provides the $P$ value. The plausibility of the null hypothesis is measured with the $P$ value; the smaller the $P$ value, the less plausible the null hypothesis. Essentially, the $P$ value is the probability of obtaining the data set, or worse (one that has less correlation to the null hypothesis), when the null hypothesis is true [20]. In this model, a $P$ value of 0.05 or less is used as the level of significance. 
47

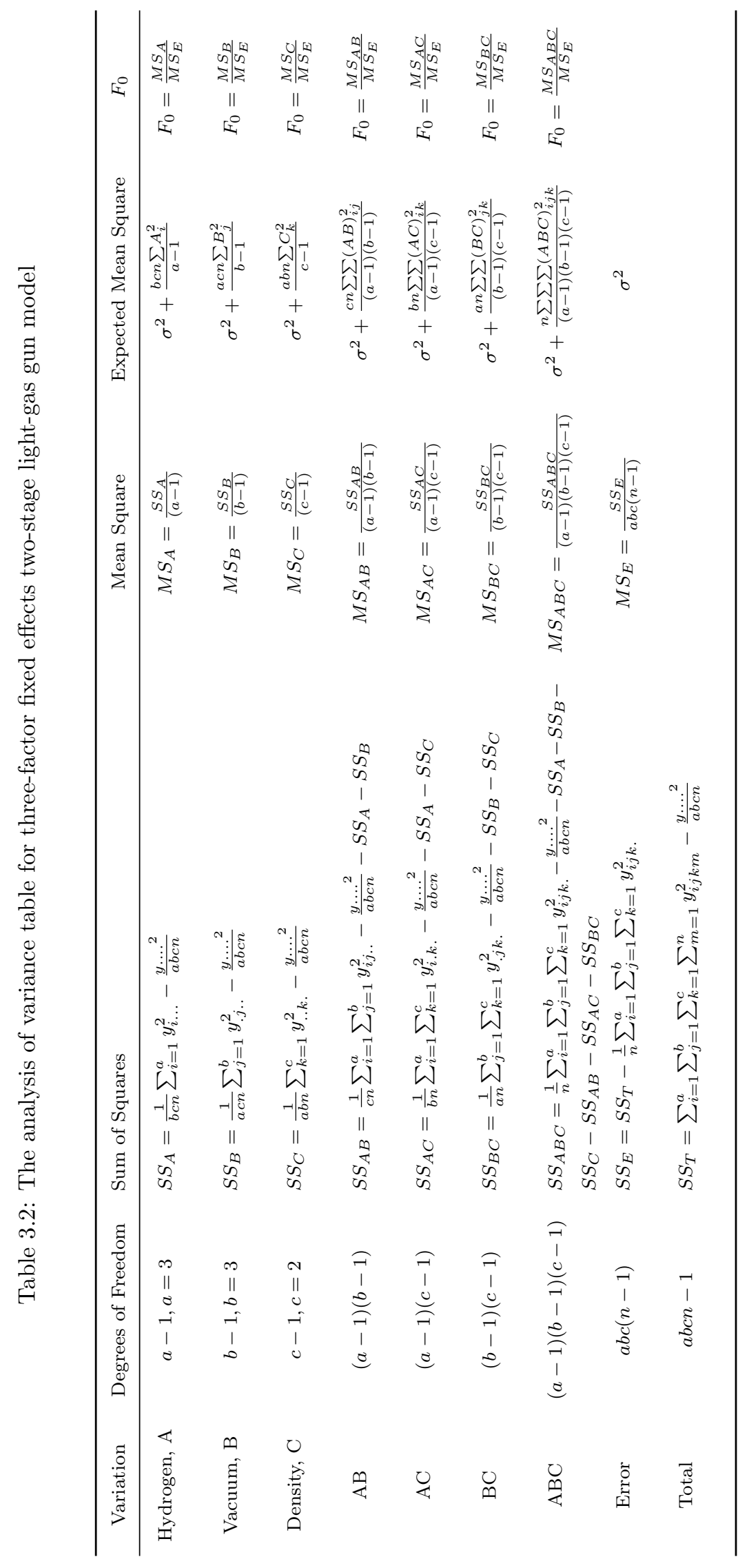


To provide more detail on the calculations in the ANOVA, the $F$-distribution can be described on the $[0, \infty)$ domain with parameters $\nu_{1}, \nu_{2}$, referred to as degrees of freedom, with the probability function

$$
f\left(y \mid \nu_{1}, \nu_{2}\right)=\frac{\Gamma\left(\frac{\nu_{1}+\nu_{2}}{2}\right)}{\Gamma\left(\frac{\nu_{1}}{2}\right) \Gamma\left(\frac{\nu_{2}}{2}\right)}\left(\frac{\nu_{1}}{\nu_{2}}\right)^{\nu_{1} / 2} y^{\frac{\nu_{1}}{2}-1} \frac{1}{\left(1+\frac{\nu_{1}}{\nu_{2}} y\right)^{\frac{1}{2}\left(\nu_{1}+\nu_{2}\right)}}, y \geq 0
$$

where $\Gamma$ is the Gamma function defined as

$$
\Gamma(n)=\int_{0}^{\infty} x^{n-1} e^{-x} d x
$$

Consider two independently distributed chi-square random variables $y_{1}$ and $y_{2}$ representing variables in the model with $\nu_{1}$ and $\nu_{2}$ degrees of freedom. The corresponding $F$ statistic is consequently

$$
F_{0}=\frac{\frac{y_{1}}{\nu_{1}}}{\frac{y_{2}}{\nu_{2}}}=\frac{y_{1}}{y_{2}} \times \frac{\nu_{2}}{\nu_{1}}
$$

which is $F$-distributed with $\nu_{1}$ and $\nu_{2}$ degrees of freedom [36].

Under the null hypothesis that changes in the levels of a factor have no effect on the velocity response, the quantity

$$
F_{0}=\frac{M S_{f}}{M S_{e}}
$$

where

$$
\begin{aligned}
& M S_{f}=\text { Mean square for the factor under consideration } \\
& M S_{e}=\text { Mean square for error }
\end{aligned}
$$

will be $F$-distributed with degrees of freedom corresponding to degrees of freedom of each mean square. Consequently for a certain calculated $F_{0}$, the corresponding $P$ value is determined by

$$
\int_{0}^{F_{0}} f\left(y \mid \nu_{1}, \nu_{2}\right) d y=1-P
$$

\subsection{Full-Factorial Results}

The DOE was analyzed with MINITAB ${ }^{3}$ and results are listed in Table 3.3. Prior to the DOE, it was hypothesized that the projectile velocity could potentially be adjusted by varying the amount of hydrogen pressure in the pump tube, the amount of vacuum or atmosphere present in the flight tube and target chamber, and/or using a different geometry and mass (hence density) launch package;

\footnotetext{
${ }^{3}$ All ANOVA calculations done with MINITAB, Release 15, 2007, www.minitab.com
} 
Table 3.3: The analysis of variance table for three-factor fixed effects gas-gun experiment

\begin{tabular}{clcccc}
\hline Variation & DOF & Sum of Squares & Mean Square & F Value & P Value \\
\hline Hydrogen Pressure & 2 & 0.356 & 0.178 & 0.18 & 0.839 \\
Vacuum Level & 2 & 0.632 & 0.316 & 0.31 & 0.734 \\
Launch Package Density & 1 & 139.575 & 139.575 & 138.76 & 0.000 \\
Hydrogen-Density & 2 & 0.057 & 0.028 & 0.03 & 0.972 \\
Vacuum-Density & 2 & 0.603 & 0.302 & 0.30 & 0.745 \\
Hydrogen-Vacuum & 4 & 1.749 & 0.437 & 0.43 & 0.782 \\
Hydrogen-Vacuum-Density & 4 & 2.333 & 0.583 & 0.58 & 0.681 \\
Error & 18 & 18.106 & 1.006 & & \\
Total & 35 & 163.411 & & & \\
\hline Standard Deviation $=1.00294$ & $R^{2}=88.92 \%$ & & & \\
\hline
\end{tabular}

yet the actual extent to which this was true was unknown. For example, it was previously speculated that the velocity of the launch package could be increased by increasing the level of hydrogen pressure in the pump tube, closer to $1.4 \mathrm{MPa}$, and setting a lower level of vacuum, near $0.14 \mathrm{kPa}$. This assumption was based on the idea that the projectile would receive a stronger shock wave to initiate its movement from higher levels of hydrogen pressure, and would experience less drag during free-flight from the decreased amount of air molecules present in a lower vacuum level. However, these assumptions proved to be false. The only factor that produced a statistically significant impact on velocity was the launch package density. In fact, its P-value is so much less than the other factors or combination and interaction of the other factors, it can be said that it is the single driving variable on which hypervelocity depends (within the safe envelope of operation investigated by this DOE).

To provide an example of interpretation and understanding the MINITAB results, consider the second row of Table (3.3). The $M S_{f}$ for the factor 'Vacuum Level' is 0.316 with 2 degrees of freedom while the $M S_{e}$ for error is 1.006 with 18 degrees of freedom. Therefore the ratio is 0.314 , and if the $\mathrm{F}$ distribution were integrated using the probability function

$$
\int_{0}^{0.314} f(y \mid 2,18) d y=0.266
$$

This integral produces a $1-P$ value of 0.266 , providing a $P$ in the ANOVA table of 0.734 . Since this value is well above the 0.05 level of significance set, the factor is not a rare event based on the experimental results. In other words, the factor of vacuum level alone does not create a statistically significant effect on resulting velocity. 


\subsection{Validation of ANOVA Model}

In order to have confidence in the DOE analysis, the assumptions of the ANOVA model must be met. The three assumptions of an ANOVA model are that:

1. The values for each level are normally distributed (Gaussian).

2. The observations are independent.

3. The variances are the same for each level (Homogeneity of Variance).

In order to help validate the model, a normal distribution plot is examined. Figure 3.4.1 plots the normal probability distribution of the residuals. The residuals are the difference between the fitted model and the data points. The ANOVA model assumes that the residuals are normally distributed with a mean of zero. The blue line is the best fit normal distribution to the data points with a forced zero fit at the $50 \%$ point. The independent axis is the normal distribution percentage while the dependent axis is the actual deviations. Recall that the error standard deviation was 1.003 (from the eighth row of results in Table 3.3) and this is reflected in the values on the independent axis. As can be seen from Figure 3.4.1, the model appears to be well-behaved and helps support the first two model assumptions.

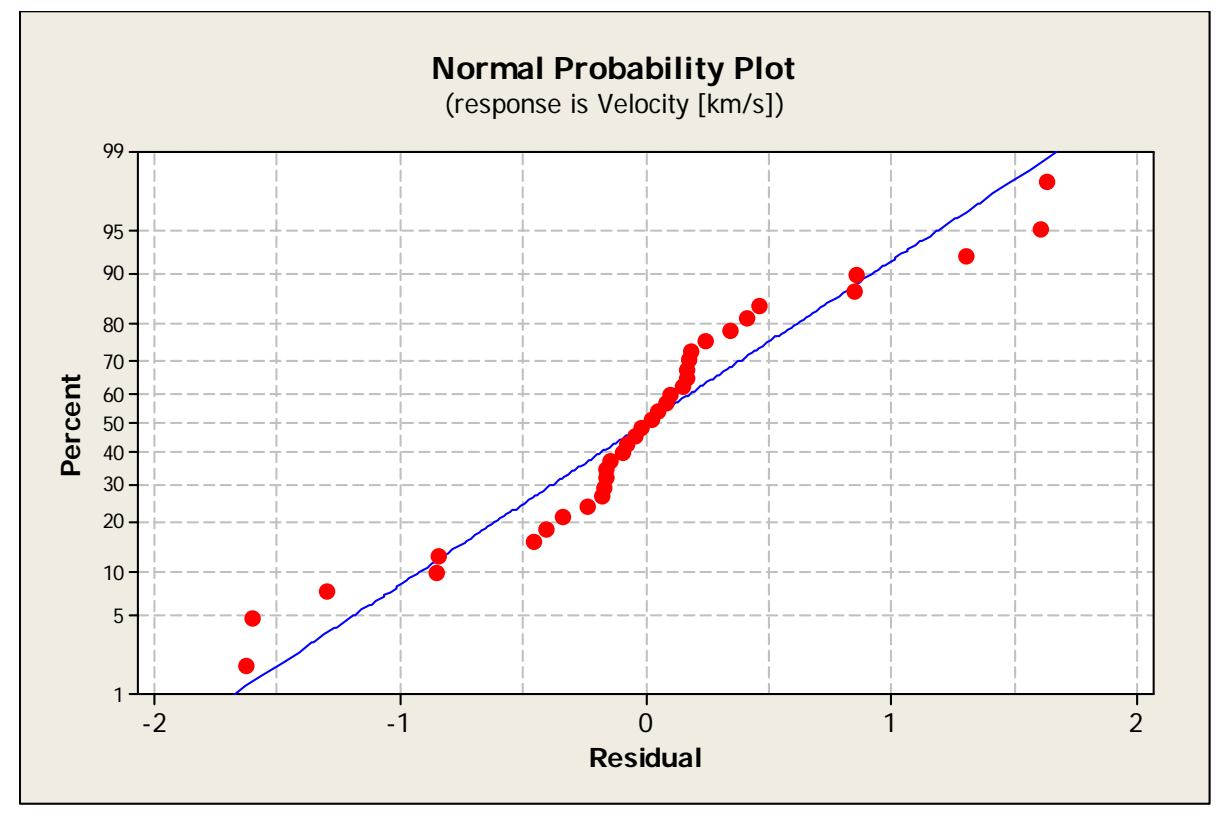

Figure 3.4.1: Normal probability plot of residuals in two-stage light-gas gun ANOVA DOE 
Figure 3.4.2 illustrates a run chart of the residuals, or the residuals as a function of observation order. There appears to be no pattern to the run chart, so the residuals are most likely mutually independent. This means there there was no discernible systemic error or unknown influence on the DOE. The residuals also appear to be homoscedastic with respect to run order. As a result, the third assumption of the ANOVA model does not appear to be violated since the observations seem independent.

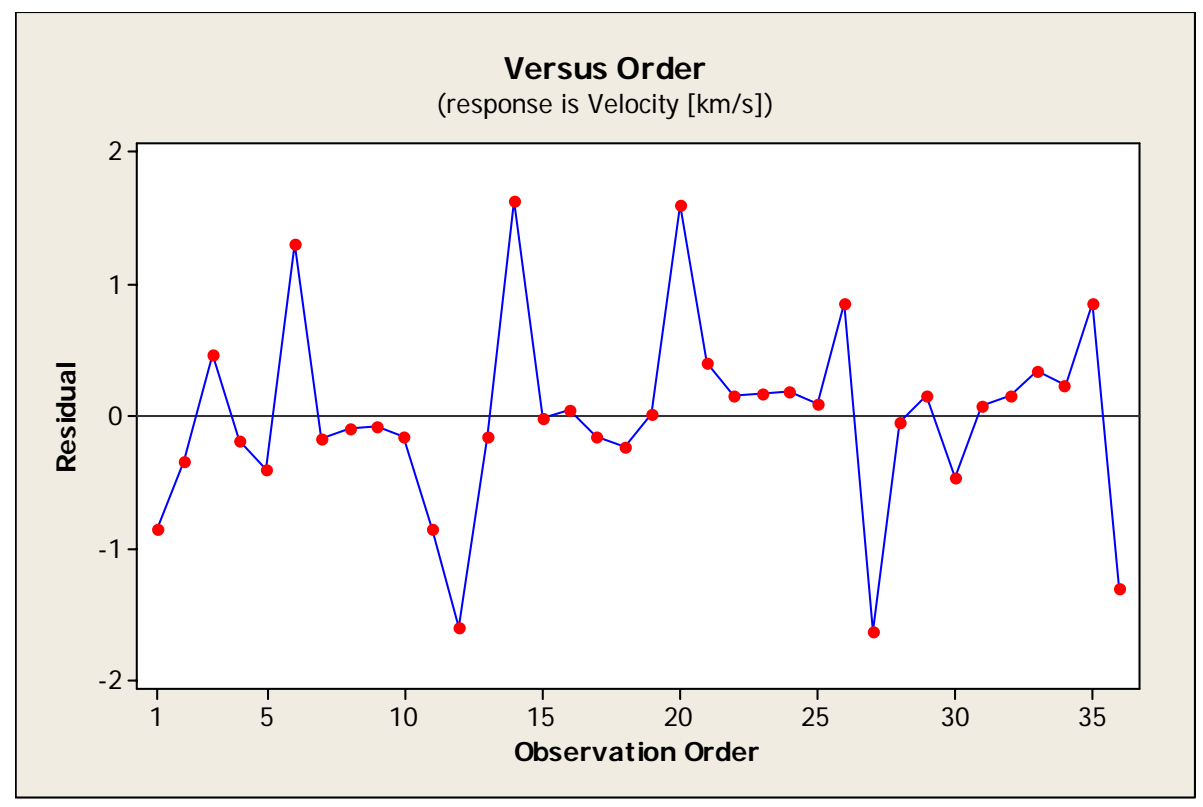

Figure 3.4.2: Residuals versus the order of the recorded observations in two-stage light-gas gun ANOVA DOE

In this particular DOE, the only factor that needs the equality of variances examined in the different samples (validating assumption 3) is launch package density, since both hydrogen pressure and vacuum level factors were determined to have no significant effect on impact velocity. Therefore an F-test is performed in MINITAB to determine if the standard deviations of the two launch package density populations are equal. A two-tailed test is used with the $\mathrm{F}$ hypothesis defined with the null hypothesis, $H_{0}$ as:

$$
\sigma_{1}=\sigma_{2}
$$

stating that the variances are equal, and the alternate hypothesis, $H_{a}$ as

$$
\sigma_{1} \neq \sigma_{2}
$$

stating that the variances are not equal [48]. 
Figure 3.4.3 illustrates the homogeneity of variance from these two tests used for determining homoscedasticity. The only factor that is considered is launch package density since both vacuum level and hydrogen pressure factors exhibited no significant effect on velocity. However, both the F-test and Levene's test show an unequal variance across the samples, and the homogeneity of variance is not achieved. A more detailed description of the F-test and Levene's test is in Appendix A.

In addition, the F-test results examine if the standard deviations of two populations are equal. Again, it looks as though the model exhibits some heteroskedascity, since the distributions are uneven in span. This suggests that perhaps the second assumption of the ANOVA model is violated and should be examined further.

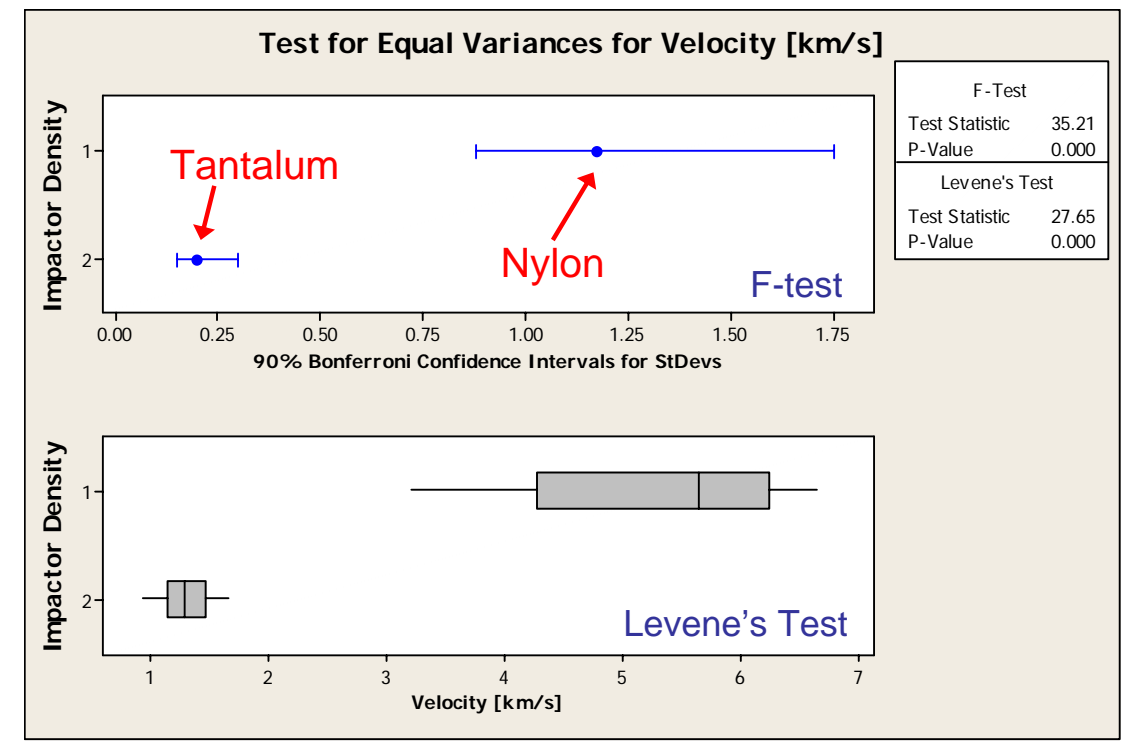

Figure 3.4.3: F-test and Levene's Test to examine ANOVA model equivalence of variance

To examine the second assumption of an ANOVA model further, Figure 3.4.4 shows a plot of the residuals versus the predicted values. The amount of variation in the residuals appears to be uneven depending on whether the magnitude of the response is large or small. This again suggests that the model is heteroskadastic in nature. Essentially, the residual versus fitted plot shows that the ANOVA model fits tantalum well (more dense launch package), but nylon not nearly as well (less dense launch package). This variation could be for a variety of reasons involved in the actual mechanics of a launch. For instance, the scatter could be due to the fact that the nylon is not a perfectly symmetric geometrical shape like the tantalum spheres and exhibits tumble down the flight tube, or because the nylon is a polymer unlike the crystalline metallic tantalum and could exhibit areas of rapid melting and vaporization due to friction along the launch tube walls before it enters free-flight. 


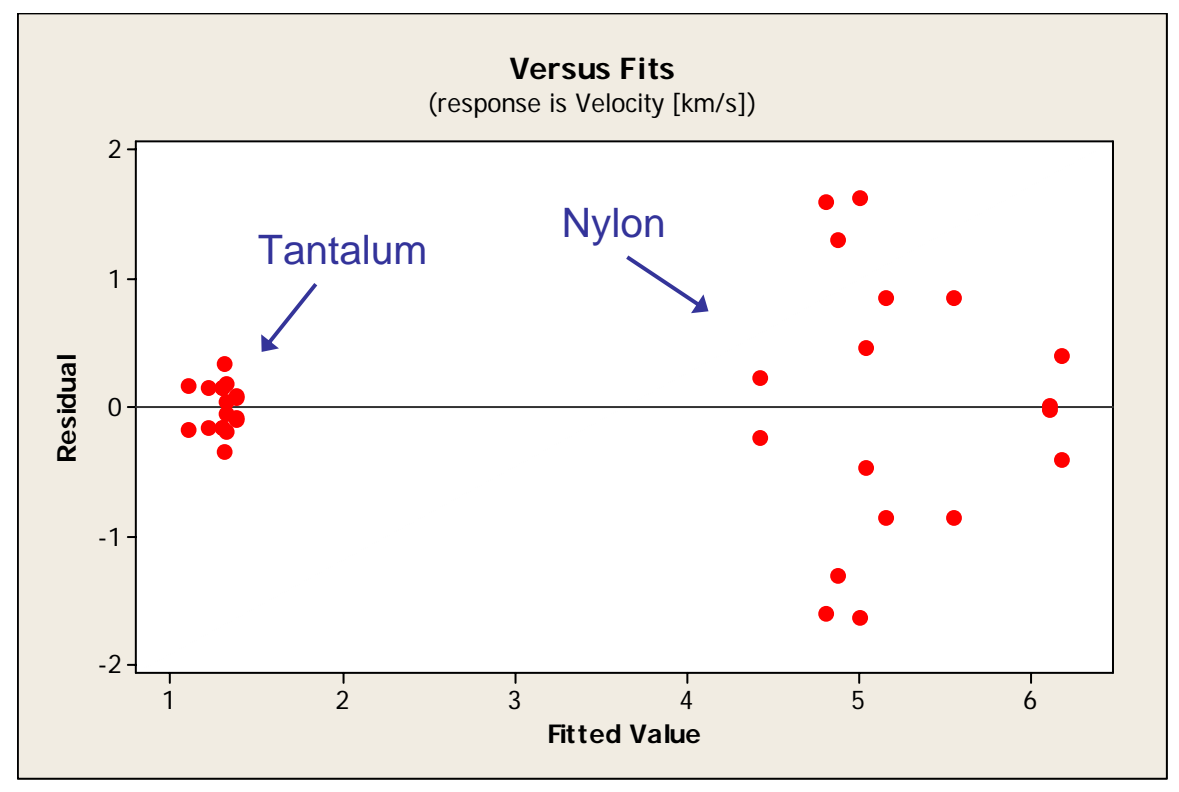

Figure 3.4.4: Residuals versus fitted values

Plotting residuals versus the value of a fitted response should produce a distribution of points scattered randomly about 0 , regardless of the size of the fitted value. In this case, however, residual values seem to increase as the size of the fitted value increases. When this happens, the residual cloud becomes 'funnel shaped' with the larger end toward larger fitted values; that is, the residuals have larger and larger scatter as the value of the response increases. This again suggests that the second assumption of the ANOVA model is violated. If a rigorous analysis of the yield variable were needed, a transformation could be performed to make the variances equal. However, in this case since the only significant factor on velocity is the launch package density, the analysis can be further split to check the validity of the model on each launch package separately. Moreover, the ANOVA model is relatively insensitive to deviations in the condition of homoskedasticity [32].

\subsection{Interactions}

The presence of interactions compromises interpretation of main effects, depending on the magnitude of interaction. Interaction plots depict the levels of one factor across the horizontal axis, with the vertical axis being the units of response. In this case, Figure 3.5.1 illustrates the potential interactions between the three factors of hydrogen gas pressure, vacuum level, and launch package density on the outcome of velocity in the gas-gun facility. One possible interaction exists between the amount 
of hydrogen gas and vacuum level as can be seen by the crossing lines, however, the difference is subtle and may only be a source of random error. When compared with the launch package density, for the most part the lines of varying hydrogen gas pressures and vacuum levels appear on top of one another, again suggesting that they play a negligible role, even coupled, on the yield variable.

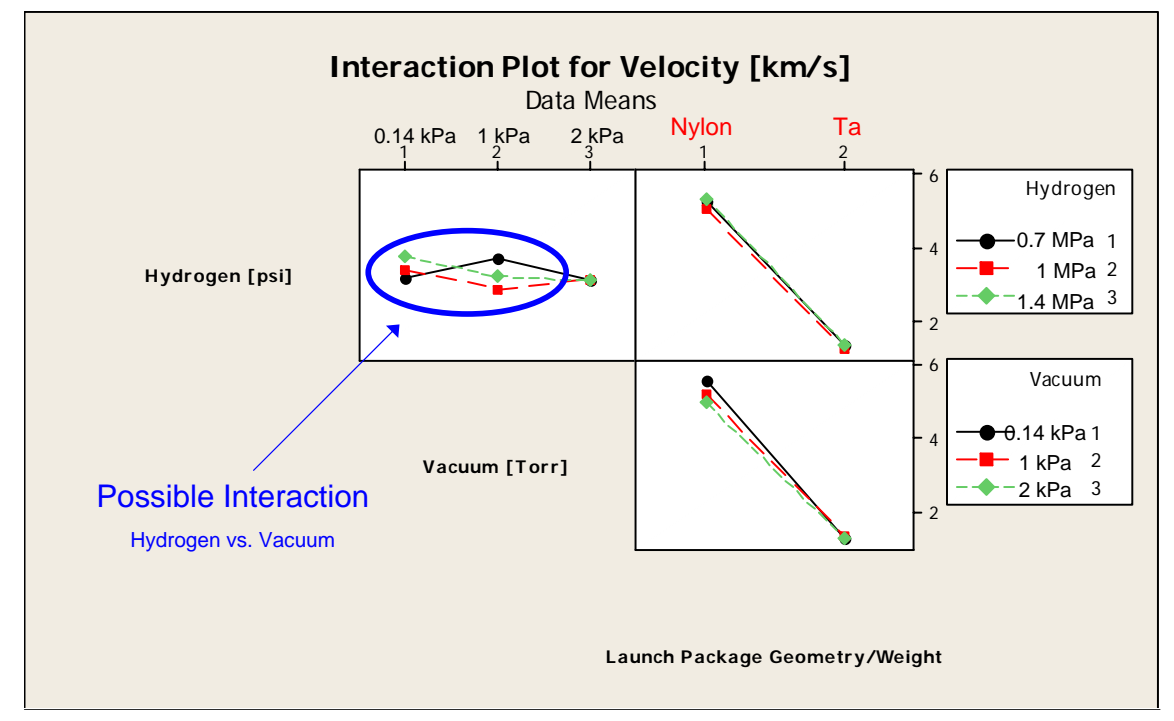

Figure 3.5.1: Factor interaction plot of gas-gun ANOVA DOE

\subsection{First-Order Hypervelocimetry Model}

Now that it has been established that the internal ballistics factor of hydrogen pressure, and external ballistics factor of vacuum level, or any interaction of the two, has no statistically significant role in the yield variable, velocity, a simple regression model on the DOE data can be determined. This expression provides a first-order approximation of the launch package velocity as a function of its mass (derived from its density). The ballistic factors are free to be modified for other benefits, such as enhancing certain diagnostics, without affecting the impact velocity. For example, to utilize some visualization techniques of the launch package, it would be advantageous to run a test at a higher vacuum level in order to have more air molecules present in the target chamber and create a brighter plasma sheath around the launch package to capture using high-speed photography.

Furthermore, tests that were run with different density launch packages, regardless of the other test conditions, can be added to the first-order model to improve it. In this case, approximately 20 more tests run at various hydrogen and vacuum levels using launch packages of 440C stainless steel spheres, each $1.8 \mathrm{~mm}$ in diameter and roughly $23 \mathrm{mg}$ in mass, were added into the initial first-order 


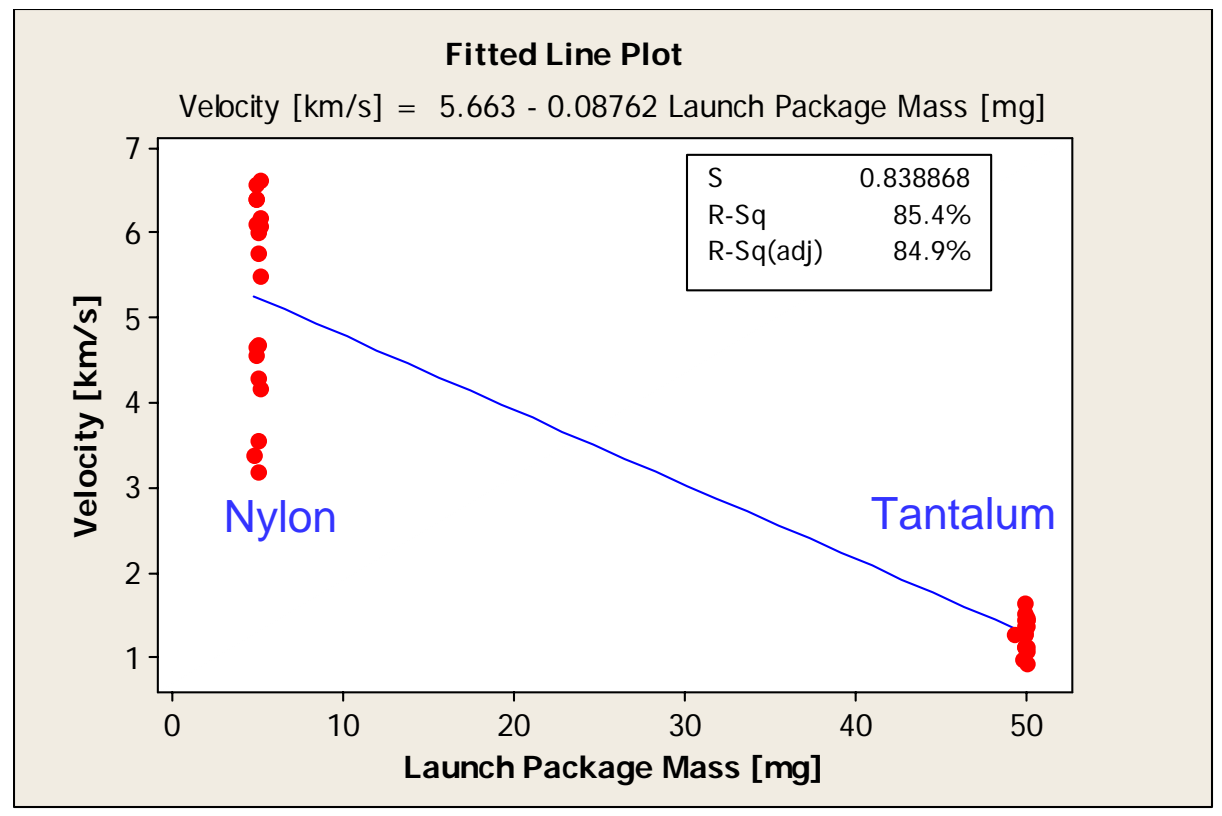

Figure 3.6.1: Fitted line plot of DOE data, mass of launch package versus resulting velocity

model. As a result, Figure (3.6.2) shows the relation between hypervelocity and launch package mass is actually not a linear one, but rather grows in a more quadratic fashion.

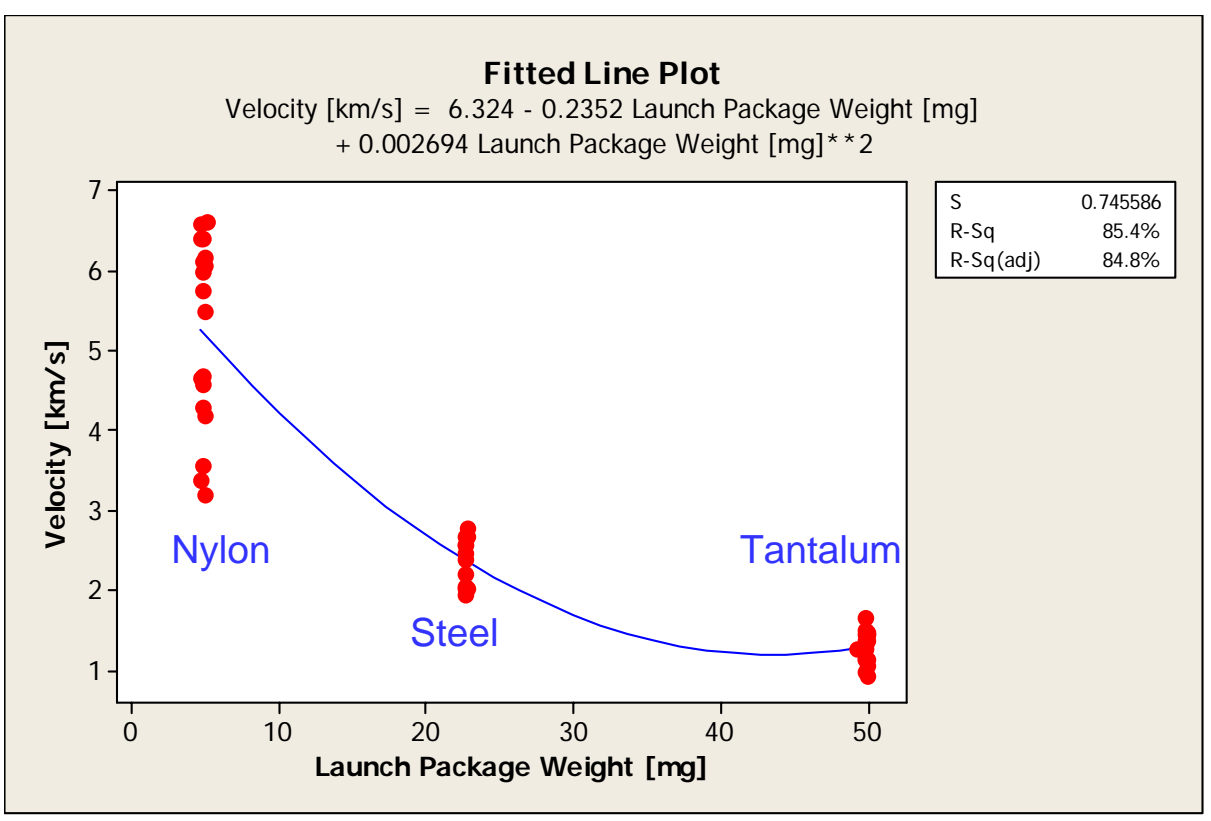

Figure 3.6.2: Regression analysis, fitted curve to extended DOE data, mass of launch package versus resulting velocity 


\subsection{Conclusions \& Recommendations}

A complete full-factorial analysis of variance design of experiments was conducted on the two-stage light gas gun used in empirical investigations. The unique facility had never previously been systematically studied to obtain the effect of internal and external ballistic factors on maximizing velocity. It was discovered that out of the three factors examined, hydrogen gas pressure, vacuum level, and launch package density, only launch package density had a statistically significant influence on the yield variable of velocity.

Additionally, the validity of the assumptions in the ANOVA model were examined. The normal probability distribution plot of the residuals verified that the values for each level are normally distributed. Moreover the plot of the residuals versus the order of the data verified that there was no appreciable systematic error and that the observations were mutually exclusive. Lastly, the assumption of equal variance of each level appeared to be violated, as was indicated in the Levene's and F-test plots. This heteroskadastic tendency was further noted by the plot of residuals versus fitted values as there appeared to be significantly larger scatter for nylon density launch packages than for tantalum density launch packages. A few potential physical reasons for this unequal variance were suggested. A possible transformation could be performed to force homogeneity of variance, however because the ANOVA model is relatively insensitive to deviations in the condition of homoskedasticity, this was not pursued.

It should be noted that only the first level of internal and external ballistic variables were considered in the DOE. That is, only factors that were at a first-level means of altering in a launch. Consequently, one of the recommendations for future work in statistical analysis of hypervelocimetry on the gas-gun would be to consider another, less straightforward, set of factors. Some of these include the amount of gun powder used, size of AR section, size of piston, or use of lighter launch packages in the form of a sabot. However, these types of variables have limits that are dictated less by safety and more by the physical mechanics of the light-gas gun. The limit of gun powder and size of AR section are coupled in that you can only increase gun powder until either you damage the action or you completely push the piston out of the converging end of the AR section and create a dirty shot downrange. In any case, the results of the ANOVA DOE provided a first-order approximation of estimating gas-gun velocity depending on the density (or more directly mass) of the launch package used. 


\section{Chapter 4}

\section{Terminal Ballistics}

In the case of spacecraft design and shielding, the ballistic limit describes the velocity threshold where a specific diameter shield just stops a given size orbital debris or meteoroid from perforation or shield failure. Numerous thin plate perforation (or hole prediction) equations have been developed over the years, predominantly through testing at various NASA centers [22]. The majority of relationships are empirical in nature, developing expressions to determine the diameter of the hole in a thin sheet subjected to hypervelocity impact based on various geometrical and material properties. A consequence of this method of developing perforation expressions is that, the models are often unreliable when used on different materials or geometries, which means they are lacking some of the underlying physical parameters that govern hypervelocity impact damage. A few of the current and historical models will be reviewed briefly, and then compared to some of the thin plate perforation experiments performed on the two-stage light-gas gun facility.

\subsection{Ballistic Limit Equations}

One of the early physically based models for plate perforation was developed by Rolsten in 1964 for spherical projectiles impacting a thin target plate with no obliquity [51]. In this approach, the impactor and plate material that come in contact are assumed to behave like a fluid. Radial flow of the impactor is assumed to stop when an equal volume of plate material has been displaced. Using this hydrodynamic approach to perforation, a hole diameter is said to equal the displaced quantity [51]. Using Bernoulli's assumption at a steady state condition, the expression for stagnation pressure, $\mathrm{P}_{0}$, in terms of the impact velocity, $\mathrm{v}_{i}$, perforation velocity, $\mathrm{v}_{p}$, target density, $\rho_{t}$, and impactor density, $\rho_{i}$ is

$$
P_{0}=1 / 2 \rho_{t} \mathrm{v}_{p}^{2}=1 / 2 \rho_{i}\left(\mathrm{v}_{i}-\mathrm{v}_{p}\right)^{2}
$$


and rearranging this expression gives

$$
\frac{\mathrm{v}_{i}}{\mathrm{v}_{p}}=\left(\frac{\rho_{i}}{\rho_{p}}\right)^{1 / 2}+1
$$

Assuming the length of the impactor is $x_{i}$ and is consumed at any time increment, $d t$, by

$$
d x_{i}=\left(\mathrm{v}_{i}-\mathrm{v}_{p}\right) d t
$$

the depth of penetration into the target can be described as

$$
d x_{t}=\mathrm{v}_{p} d t
$$

Let $d Q_{i}$ be the volume of incremental impactor consumed, $d Q_{t}$ be the volume of incremental target removed from impact, and $d Q_{H}$ be the volume of ultimate target material removed and is expressed as

$$
d Q_{i}+d Q_{t}=d Q_{H}
$$

For an initial impactor radius, $R_{0}$, and initial impactor diameter, $D_{0}$, the volume of the impactor consumed can be written

$$
d Q_{i}=\pi R_{0}^{2} d x_{i} d Q_{i}=\int_{0}^{t} \pi R_{0}^{2}\left(\mathrm{v}_{i}-\mathrm{v}_{p}\right) d t
$$

and the volume of target material consumed is

$$
d Q_{t}=\int_{0}^{t} \pi R_{0}^{2} \mathrm{v}_{p} d t
$$

If an annular segment of the target is removed

$$
d Q_{H}=2 \pi \int_{R_{0}}^{R} R d R \int_{0}^{t} \mathrm{v}_{\mathrm{p}} d t
$$

this gives

$$
R^{2}=R_{0}^{2}\left[1+\left(\frac{\mathrm{v}_{i}}{\mathrm{v}_{p}}\right)\right] .
$$

Using 4.1.9, substituting the expression 4.1.2, and solving for diameter, the final expression becomes

$$
D=D_{0}\left[2+\left(\frac{\rho_{t}}{\rho_{p}}\right)^{\frac{1}{2}}\right]^{\frac{1}{2}} .
$$

One of the most significant limitations of the Rolsten model is that is does not take impact velocity into account, which has been strongly suggested to relate to perforation diameter [22]. 
The other physically derived perforation prediction model for thin plates was developed by Charters in 1959 using principles from mechanics [45]. In this case it was assumed that $1 / 2$ the incoming kinetic energy is used to expand a fluid shell around the impact site, and is related to the target plate deformation stress $S$ [45] by

$$
\int_{0}^{D} S \times 2 \pi D^{2} d D=\frac{1}{4} m \mathrm{v}^{2}
$$

where $D$ is the diameter of the impact penetration, $m$ is the mass of the impactor, and $\mathrm{v}$ is the incoming collision velocity. This corresponds to

$$
S=\frac{3}{8} \pi\left(\frac{m \mathrm{v}^{2}}{D^{3}}\right)
$$

and by converting the impactor mass to density and volume of a sphere of diameter $D$, leads to the equation

$$
D=\frac{1}{2}\left(\frac{\rho_{p} \mathrm{v}^{2}}{2 S}\right)^{\frac{1}{3}}
$$

One of the limitations of the Charters model is that the strength required to make the equation fit the data is many times the static strength and it has been observed that increased material strength does not necessarily decrease the penetration hole size [45].

Most of the other existing perforation equations are empirically or semi-empirically based. A few of the more traditional expressions include the Maiden model, developed from data on spherical aluminum projectiles impacting aluminum targets [33], which is given as

$$
D=D_{p}\left[2.4 \frac{\mathrm{v}}{c}\left(\frac{t_{t}}{D_{p}}\right)+0.9\right]
$$

where $D$ is the penetration hole diameter, $D_{p}$ is the impactor diameter, $\mathrm{v}$ is impact velocity, $c$ is longitudinal wave speed of the target, and $t_{t}$ is target thickness.

The Ames equation was empirically developed for thin semi-infinite solids and corresponds to

$$
D=D_{p}\left(2.6\left[\left(\frac{\rho_{p}}{\rho_{t}}\right)\left(\frac{\mathrm{v}}{c}\right)\right]^{0.22}\left(\frac{t_{t}}{D_{p}}\right)^{\frac{2}{3}}+1.0\right)
$$

where $D$ is the penetration hole diameter, $D_{p}$ is the impactor diameter, v is impact velocity, $c$ is the longitudinal wave speed of the target, $\rho_{p}$ is the impactor density, $\rho_{t}$ is the target density, and $t_{t}$ is target thickness [22]. 
Sawle developed an alternative model to the Ames equation based on target shear strength considerations developed by Sorenson [45] expressed as

$$
D=D_{p}\left[\left(\frac{\rho_{p}}{\rho_{t}}\right)^{0.055}\left[\rho_{p}\left(\frac{\mathrm{v}^{2}}{\sigma_{U S}}\right)\right]^{0.1}\left(\frac{t_{t}}{D_{p}}\right)^{\frac{2}{3}}+1.0\right]
$$

where $\sigma_{U S}$ is the shear strength of the target material and all other variables are the same as 4.1.15 [22].

Numerous existing models, such as the Nysmith and Denardo expression [38], are not dimensionally correct, and are not considered in the discussion. In later years, computer software and optimization code, such as MULTIVAR, were used to take various databases of hypervelocity impact tests and calculate best fit coefficients, examining the resultant sum of the residuals squared for a correlation coefficient. One such MULTIVAR relationship for a spherical projectile to perforation diameter is as follows

$$
D=2.947 D_{p}\left(\frac{\mathrm{v}}{c_{p}}\right)^{0.055}\left(\frac{\mathrm{v}}{c_{t}}\right)^{0.339}\left(\frac{\rho_{p}}{\rho_{t}}\right)^{0.028}\left(\frac{t_{t}}{D_{p}}\right)^{0.359}+0.342 D_{p}
$$

with $c_{t}$ as the longitudinal wave speed of the target, $c_{p}$ as the longitudinal wave speed of the projectile, crack speed $\mathrm{v}$, target and projectile density $\rho_{t}$ and $\rho_{p}$, target thickness $t_{t}$, and diameter of the projectile $D_{p}$.

While the length and number of variable ratios has increased in the computer-generated codes, the physical basis or understanding of parameters that contribute to damage perhaps has not.

\subsection{Modified Charters Equation}

A perforation prediction equation with some physical basis is developed from a modified version of the mechanics based equation of Charters. During a hypervelocity impact, shock physics, as described in the first chapter, play a substantial role in damage. As a result, during such high-energy and high-speed impacts, ductile materials may perhaps behave in a brittle fashion, and rate-dependent materials may not exhibit their dependence as strongly. Consequently, while the Charters model uses the quasi-static shear strength of the target to dictate permanent damage, a shear stress resulting from the initial extreme pressure wave is suggested in this approach. A simplified shear-stress versus pressure plot (or longitudinal and transverse stresses) of a typical brittle material undergoing a strong compressive wave is shown in Figure 4.2.1. 


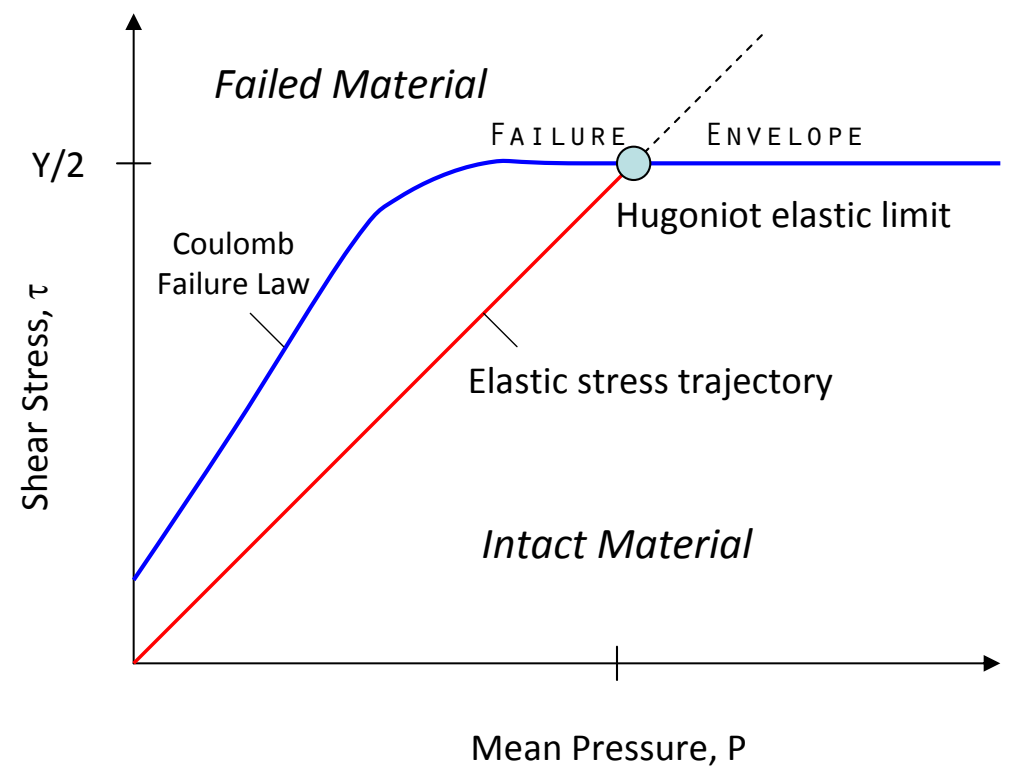

Figure 4.2.1: Plot illustrating failure from strong compressive wave with resolved shear stress for idealized brittle material

Since shear stress is a function of the longitudinal and transverse stresses, $\tau=-\left(\sigma_{L}-\sigma_{P}\right) / 2$, and the mean pressure is related to the longitudinal and transverse stresses by $P=-\left(\sigma_{L}+2 \sigma_{P}\right) / 3$, the point at which failure occurs is the intersection known as the Hugoniot Elastic Limit, $\sigma_{H E L}$ [34]. The stress at this point can be defined by the relation

$$
\sigma_{L}=-\sigma_{H E L}=-\left(\frac{1-\nu}{1-2 \nu}\right) Y
$$

and this is based off of the material properties, $Y$, the target yield stress, and, $\nu$, the target Poisson's ratio [34]. At this point, an increase in mean pressure has a constant maximum shear stress. By removing the $1 / 2$ incoming kinetic energy approximation of Charters (somewhat taken into account already by the $Y / 2$ in this model), and using the Hugoniot Elastic Limit deformation stress defined in Charters model, $\sigma_{H E L}$, the modified equation becomes

$$
\begin{array}{r}
\int_{0}^{D} \sigma_{H E L} \times 2 \pi D^{2} d D=\frac{1}{2} m \mathrm{v}^{2} \\
D=\frac{D_{p}}{2}\left(\frac{\rho_{p} \mathrm{v}^{2}}{\sigma_{H E L}}\right)^{\frac{1}{3}}
\end{array}
$$

where $D$ is the penetration hole diameter, $D_{p}$ is the impactor diameter, $\mathrm{v}$ is impact velocity, and $\rho_{p}$ is the impactor density. While this model does not take into account target thickness, it does provide a slightly better approximation based on the tests conducted using the two-stage light-gas gun facility. 
These prediction expressions are examined against tests performed with spherical impactors of tantalum and steel, $1.8 \mathrm{~mm}$ in diameter, colliding with steel targets, $2.3 \mathrm{~mm}$ thick for the tantalum impactors, and $2.6 \mathrm{~mm}$ thick for the steel impactors. Penetration hole diameters were measured by an Olympus SZ61 Microscope using Image ${ }^{1}$ software to create a mask and calculate the average hole area to an uncertainty of $\pm 3 \%$. In some cases, multiple tests had nominally equivalent impact velocities, so the average value of the resulting penetration hole diameter was used. While the Charters model provides a slightly better fit when the impactor and target plate are of the same material (steel), the modified Charters equation developed with the Hugoniot Elastic Limit, is a closer match when the impactor and target materials are different in the case of the tantalum on steel tests. One final important matter to note is that, in all of these perforation prediction equations, the differentiation between penetration and perforation is not made.

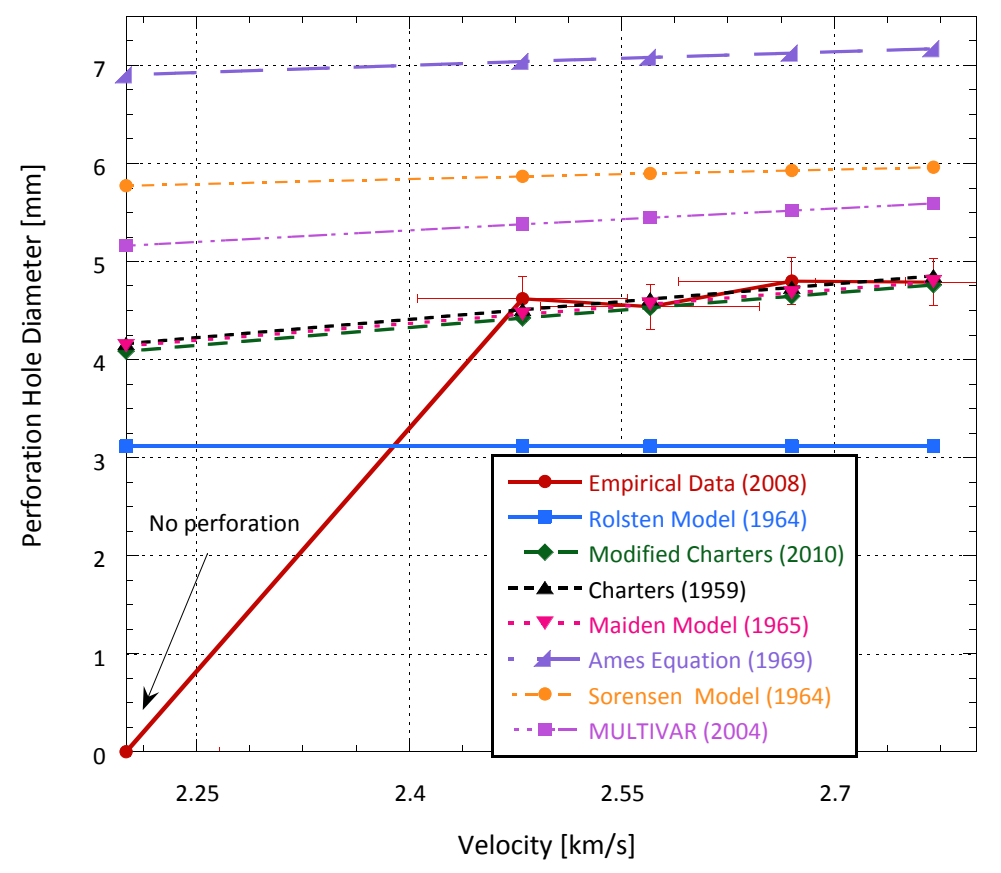

Figure 4.2.2: 440C steel sphere perforation results from impact with 304 stainless steel plates $150 \mathrm{~mm} \times 150 \mathrm{~mm} \times 2.6 \mathrm{~mm}$ at velocities between 2 and $3 \mathrm{~km} / \mathrm{s}$

\footnotetext{
${ }^{1}$ Image J software, developed by the National Institute of Health, public domain, downloaded 2009
} 


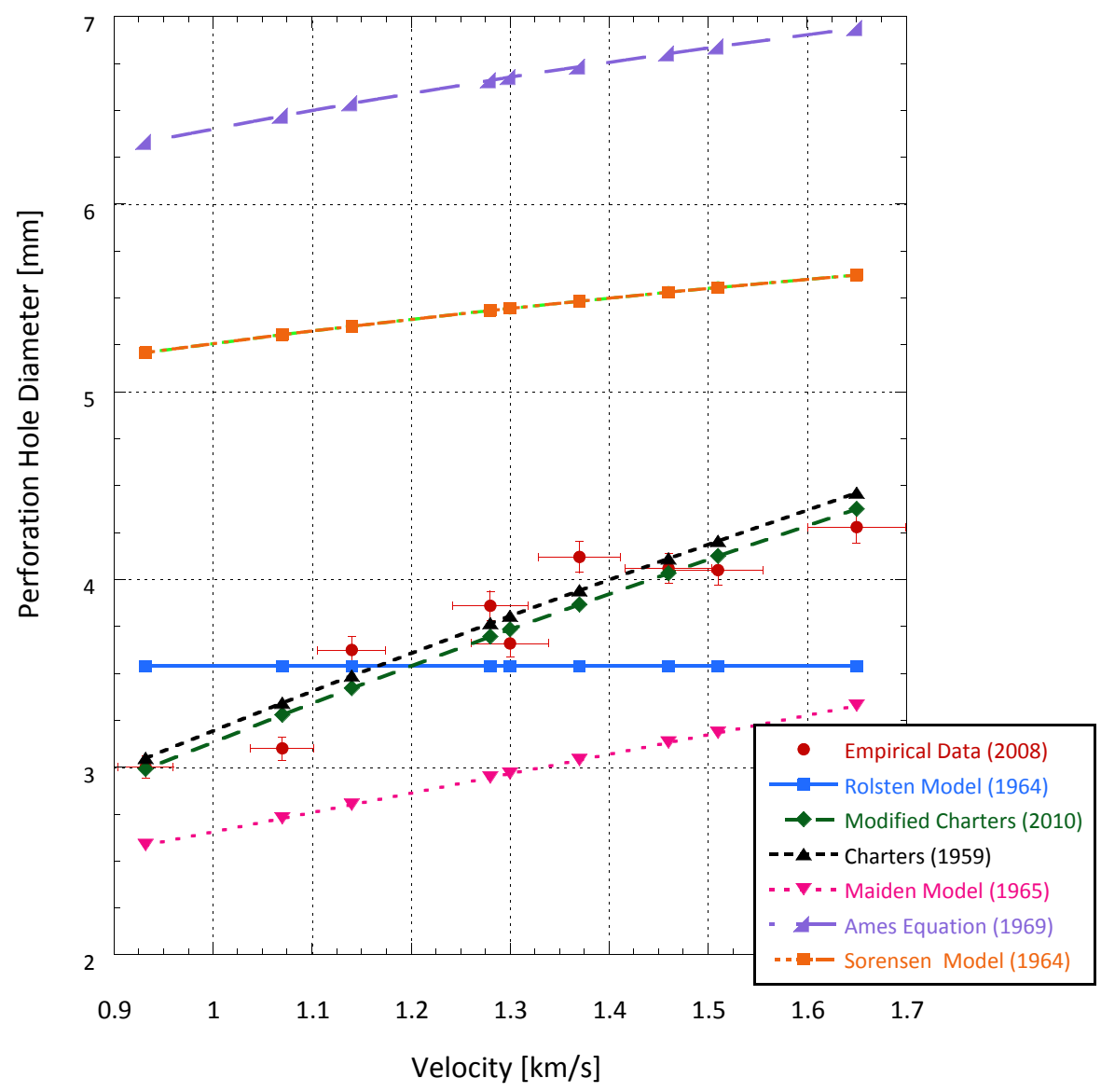

Figure 4.2.3: Tantalum sphere perforation results from impact with 304 stainless steel plates $150 \mathrm{~mm} \times 150 \mathrm{~mm} \times 2.3 \mathrm{~mm}$ at velocities between 0.9 and $1.8 \mathrm{~km} / \mathrm{s}$ 


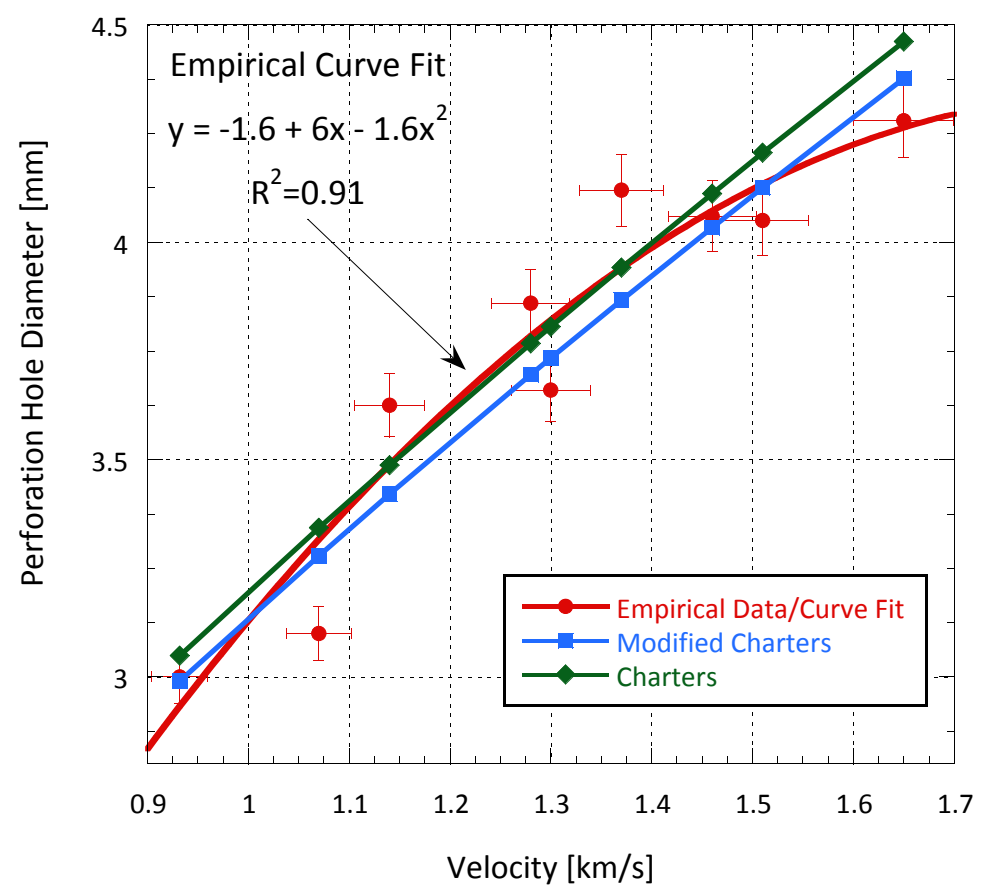

Figure 4.2.4: Charters, modified Charters, and experimental results comparison from tantalum sphere impactors, $1.8 \mathrm{~mm}$ in diameter, hole perforation size on 304 stainless steel plates, $150 \mathrm{~mm} \times 150 \mathrm{~mm} \times 2.3 \mathrm{~mm}$, at velocities between 0.9 and $1.8 \mathrm{~km} / \mathrm{s}$

While perforation prediction equations could be examined more extensively, the need to investigate hypervelocity impacts in situ, rather than post-mortem, is addressed in order to explore mechanisms of damage evolution specifically involved in dynamic fracture.

\subsection{Case Study}

Thermal protection system (TPS) materials on the current space shuttle, as well as those considered for next-generation crew vehicles, must survive intense aerodynamic heating experienced on reentry. However, little has been investigated in these materials in regards to their heat response with hypervelocity impact damage present [11]. As a result, a case study of impact damage on several raw and enhanced TPS materials in collaboration with NASA Ames Research Center, as part of the NASA Aeronatuics Scholarship Program, was conduced. All materials were impact tested at SPHIR using nylon right cylindrical slugs, $1.8 \mathrm{~mm}$ in diameter and length, between 2 and $7 \mathrm{~km} / \mathrm{s}$. Materials included: LI-2200, reusable surface insulation tile with $22 \mathrm{lbs} / \mathrm{ft}^{3}$ bulk density, Fiberform, fibrous carbon found in lightweight ceramic ablators, PICA, phenolic impregnated carbon ablators 
of various densities, as well as flexible or conformable ablators (i.e., insulation blankets) of carbon or graphite felt matrix and varying phenolic or silicone resins. Post-impact analysis included laser scanning of crater geometry on a rapid-response conoscope developed at NASA Ames, to see if any correlation between penetration geometry and density, or other material properties, exists. Density is a crucial parameter to optimize in any TPS system as it constitutes additional parasitic payload on a space structure, and decreases other aspects of performance. A possible portion of the case study considered included Arcjet testing these hypervelocity impact tested specimens to see their response to heating rates similar to atmospheric re-entry with damage present. Overall, lightweight ceramic ablators showed a decrease in impact penetration depth by a factor of roughly 1.3 with increasing carbon density as listed in Table 4.1. PICA, which was considered for the recently cancelled Orion Crew Exploration Vehicle thermal protection system, shows considerable promise in micrometeoroid and orbital debris (MMOD) protection while still maintaining a lower overall density than other traditional TPS materials.

Table 4.1: Table of thermal protection system material hypervelocity impact damage penetration response from NASA Ames case study

\begin{tabular}{ccccc}
\hline Material & No. of Tests & $\begin{array}{c}\text { Velocity Range } \\
(\mathrm{km} / \mathrm{s})\end{array}$ & $\begin{array}{c}\text { Material Density } \\
\left(\mathrm{g} / \mathrm{cm}^{3}\right)\end{array}$ & $\begin{array}{c}\text { Max Penetration Depth } \\
(\mathrm{cm})\end{array}$ \\
\hline LI-2200 & 4 & $5.8-6.7$ & 0.35 & 1.55 \\
PICA & 7 & $4.1-6.0$ & $0.28,0.38,0.48$ & 1.78 \\
Conformable Ablators & 5 & $2.6-5.2$ & $0.21,0.24$ & 2.03 \\
Fiberform & 4 & $4.9-7$ & $0.16-0.19$ & 2.62 \\
\hline
\end{tabular}




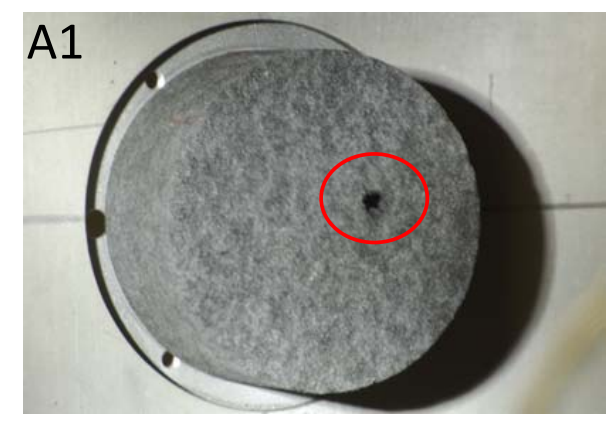

A2

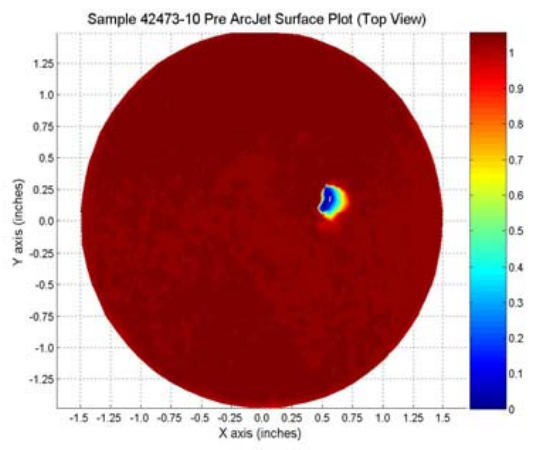

A3

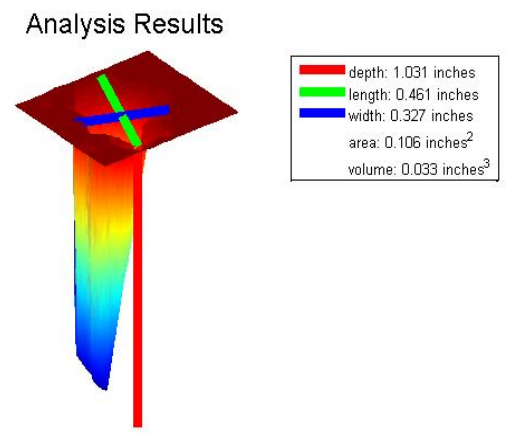

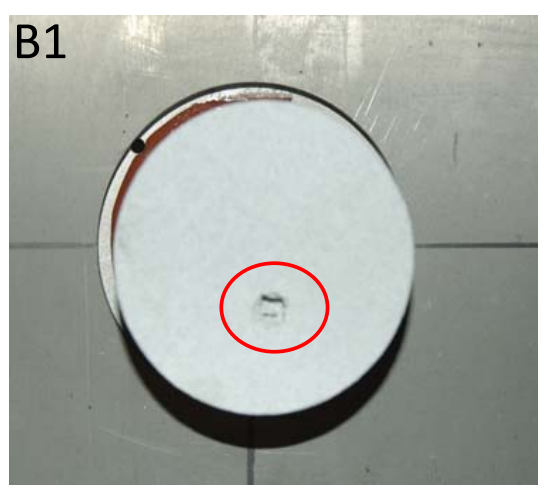

B2

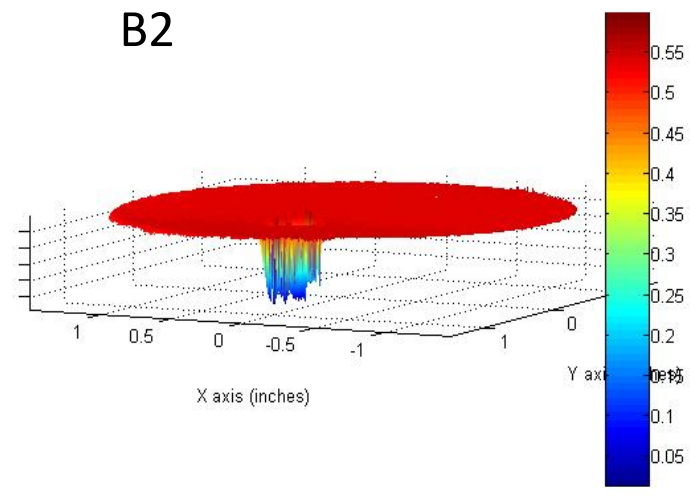

B3

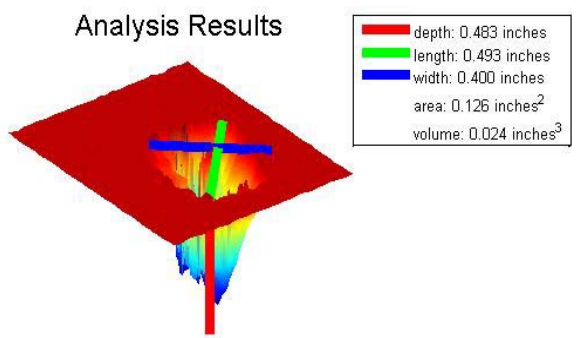

Figure 4.3.1: (A1) (Left) Fiberform cylindrical sample, $75 \mathrm{~mm}$ in diameter and $50 \mathrm{~mm}$ in length, after impact by a nylon cylindrical slug, $1.8 \mathrm{~mm}$ in diameter and length, at $7 \mathrm{~km} / \mathrm{s}$ and $0^{\circ}$ obliquity, with the corresponding penetration zone as analyzed on a conoscope (A2)(A3). (B1) (Right) LI-2200 insulating space shuttle tile cylindrical sample, $75 \mathrm{~mm}$ in diameter and $50 \mathrm{~mm}$ in length, having been impacted by a nylon cylindrical slug, $1.8 \mathrm{~mm}$ in diameter and length, at $6.1 \mathrm{~km} / \mathrm{s}$ and $0^{\circ}$ obliquity, with the corresponding penetration zone as analyzed on a conoscope (B2)(B3). Impact sites are circled in red in (A1) and (B1) 


\section{Chapter 5}

\section{Dynamic Optical Investigations}

Nominally brittle materials, such as silica glass used in the "window to the world" Cupola recently completed on the International Space Station [6], and Kapton, a Mylar-like polymer used for the tennis court-sized sunshield for the James Webb Space Telescope scheduled to launch in 2013 [2], are particularly vulnerable to dynamic crack propagation resulting from a hypervelocity impact event. Accordingly, the purpose of studying hypervelocity impacts in situ is to establish methodologies which can adequately quantify the threat of dynamic fracture resulting from a micrometeoroid or space debris impact. To date, there is no study available which concentrates on the analysis of dynamic crack growth in such thin plate or shell structures resulting in their eventual catastrophic disintegration. Therefore, the overall goal of the dynamic optical investigation is to determine the brittle material resistance to dynamic fracture in an extreme environment involving hypervelocity impacts active on thin space structures.
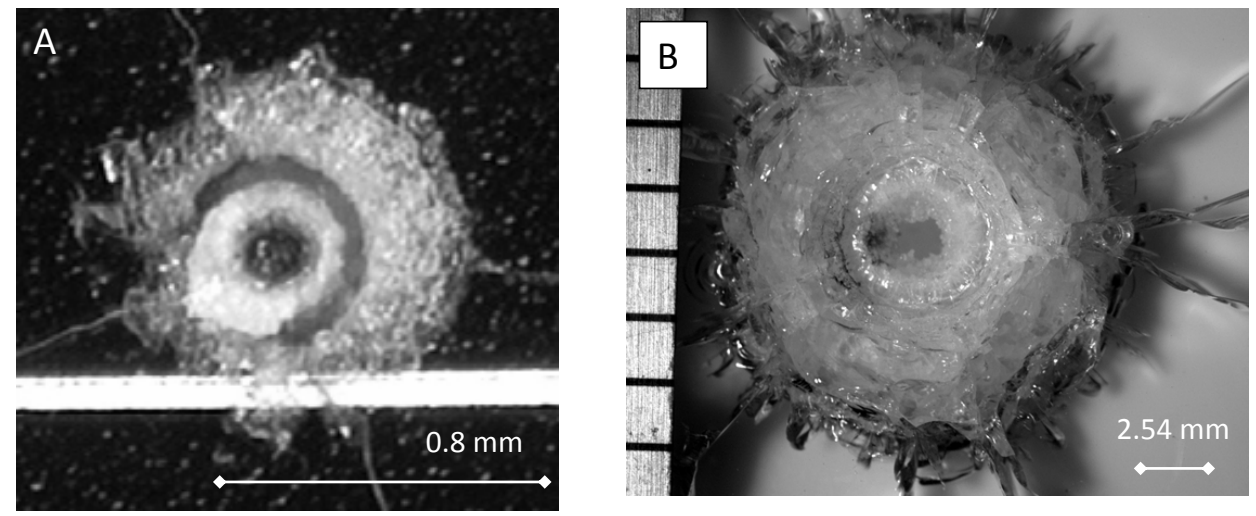

Figure 5.0.1: (A) Hubble Space Telescope solar cell hypervelocity impact damage from Drolshagen [8]. (B) Laboratory hypervelocity impact damage of Homalite 100 plate, $150 \mathrm{~mm}$ diameter and $6.4 \mathrm{~mm}$ thick, impacted by nylon slug, $1.8 \mathrm{~mm}$ length and diameter, at $5.1 \mathrm{~km} / \mathrm{s}$ 


\subsection{Dynamic Fracture Mechanics}

By assuming the validity of the principles of singular fracture mechanics in a mode-I, or crackopening mode, the stresses at the vicinity of a crack propagating with velocity $v(t)$ can be described by the singular stress field as

$$
\sigma_{i j}=K_{I}^{d} / \sqrt{2 \pi r} f(\theta, v)
$$

where in a polar coordinate system $(r, \theta)$ translating with the crack, $r$ is equal to the radial distance from the crack tip and $\theta$ defines the angular position.

The common amplitude of all stress components is the dynamic stress intensity factor $K_{I}^{d}(t)$ which varies explicitly with time as well as with the time-dependent crack tip speed, $v(t)$, and the applied loading, $P(t)$, and can be determined experimentally.

For growing cracks the dynamic stress intensity factor is

$$
K_{I}^{d}(t, v(t), P(t))
$$

and should attain a critical value, denoted as $K_{I C}^{d}$ which represents the material's resistance to dynamic crack growth.

In the case of dynamically growing mode-I cracks, this resistance is crack growth rate dependent and has been found to be a function of the crack growth speed, $v$, which is argued by Rosakis, Dally, and Kobayashi [44, 26, 9], among others, to be a material functional property and can be used in designing against catastrophic failure.

Moreover, the critical level of the energy that the surrounding material supplies to a dynamic, modeI, crack tip growing at speed $v(t)$ is called the dynamic energy release rate $G_{I C}^{d}(v)$ and can be related to the critical fracture resistance, $K_{I C}^{d}$, by

$$
G_{I C}^{d}=\frac{1}{H} A_{I}(v) K_{I C}^{2}
$$

where $A_{I}$ is a universal function of crack speed $v$, and the material's shear and pressure wave speeds, $c_{s}$ and $c_{d},[17]$ and is expressed by

$$
A_{I}=\frac{v^{2} \alpha_{d}}{(1-\nu) c_{s}^{2} B}
$$


where

$$
\begin{aligned}
B & =4 \alpha_{d} \alpha_{s}-\left(1+\alpha_{s}^{2}\right)^{2} \\
\alpha_{d} & =\sqrt{1-\frac{v^{2}}{c_{d}^{2}}} \\
\alpha_{s} & =\sqrt{1-\frac{v^{2}}{c_{s}^{2}}} .
\end{aligned}
$$

The term $H$ is a combination of the Young's Modulus, $E$, and the Poisson's ratio, $\nu$, and is given by

$$
\begin{aligned}
& H=E \text { for plane stress } \\
& H=E /\left(1-\nu^{2}\right) \text { for plane strain. }
\end{aligned}
$$

The relation (5.1.3) demonstrates that if indeed the dynamic fracture toughness, $K_{I C}^{d}$, is a material dependent function of $v$, so is the critical level of required energy, $G_{I C}^{d}$. Consequently, $G_{I C}^{d}$ is also considered to be a function characterizing the material's ability to resist fracture and can be thought of as the requisite energy dissipated at the crack tip during the dynamic fracture process [17]. One of the major goals of the study is to directly measure this function in the presence of highly energetic hypervelocity impact loading conditions.

\subsection{Experimental Configuration}

To determine the growing crack energy levels, laboratory micrometeoroid or space debris impacts were generated by hypervelocity impact to examine the dynamic fracture resistance of nominally brittle materials. In this study we were able to characterize the dynamic behavior in situ or during the impact and crack propagation events, as well as to directly observe the dominant failure modes. Thin target plates (1.6 and $3.2 \mathrm{~mm}$ ) of two different brittle polymers, Mylar and Homalite 100, were used, and as a result, plane stress conditions are expected to dominate at the vicinity of mode-I growing cracks. Each plate was $150 \mathrm{~mm}$ in diameter, and most were tested with a center hole $20 \mathrm{~mm}$ in diameter that was initially pre-cracked. The plates were then subjected to far-field quasi-static tensile loads ranging from 0.5 to $4 \mathrm{MPa}$, similar in magnitude to a functional load on a thin-walled component of a space asset, or possibly a hoop stress exhibited on a membrane or pressurized thin-walled structure of an external tank. The initial pre-cracked configuration could be thought of as an inherent material flaw, possible damage that occurred during launch, or an older component of a space asset that has pre-existing hypervelocity impact damage. A nylon 6-6 right 
cylindrical slug $1.8 \mathrm{~mm}$ in diameter and length, propelled by hydrogen gas, was shot at the target plate at velocities ranging from 3 to $7 \mathrm{~km} / \mathrm{s}(7000-15500 \mathrm{mph})$. All experiments were conducted under vacuum between 0.13 and $2.4 \mathrm{kPa}$ (1-18 Torr) and details are shown in Figure 5.2.1.

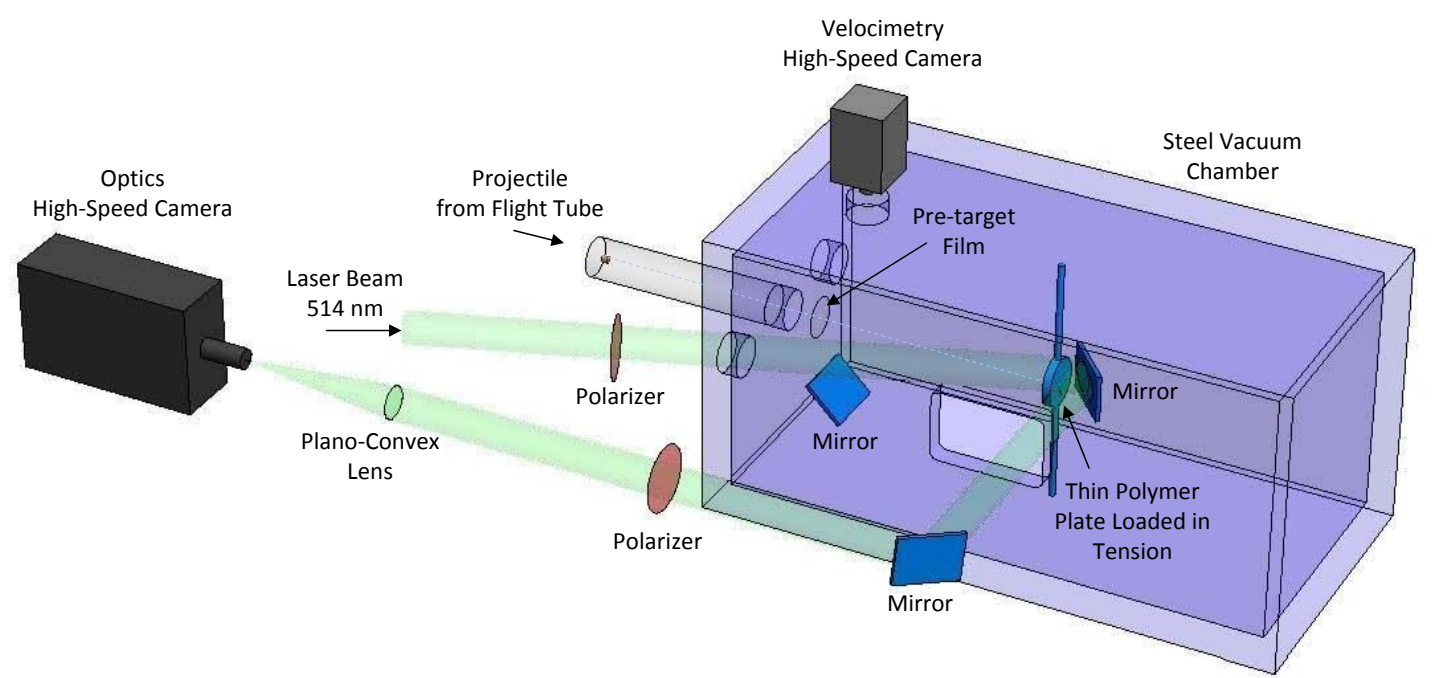

Figure 5.2.1: Dynamic optical experimental configuration using two-stage light-gas gun facility to scale [16]

A coherent, monochromatic at $514 \mathrm{~nm}$, plane polarized argon-ion laser beam was expanded to $100 \mathrm{~mm}$ and transmitted through the transparent plate specimens. A Cordin 214-8 high-speed camera resolved eight photographs of the impact stress waves, dynamic stress fields, and crack growth, triggered from a visible wavelength photodiode described in the velocimetry chapter. The lens solution consisted of a plano-convex ( $\mathrm{D}=127 \mathrm{~mm}$ diameter, $\mathrm{F}=500 \mathrm{~mm}$ focal length), circular polarizers (with quarter wave plates included), and a Pentax 67 (aperture f/2.8, F = $105 \mathrm{~mm}$ focal length) manual focus lens on the high-speed camera. 

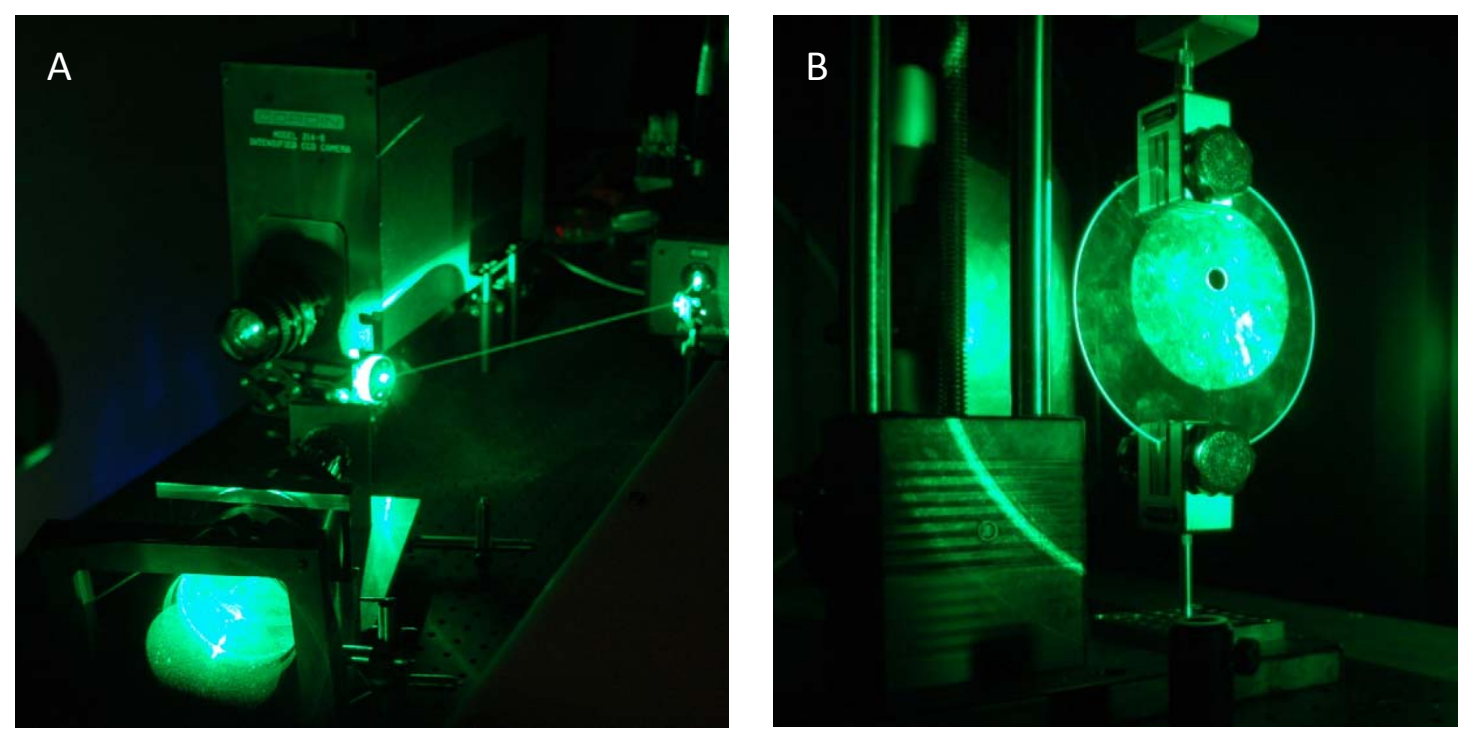

Figure 5.2.2: Photographs of the dynamic optical analysis using the two-stage light-gas gun showing (A) the optical path and (B) the target plate with initial notch held in the load frame and illuminated by the expanded laser beam

\subsection{Optical Methods}

Dynamic optical techniques were configured in transmission and combined with high-speed photography to capture 8 frames, including the complex stress wave patterns and subsequent dynamic fracture, down to the nanosecond scale. The laboratory micrometeoroids or space debris had enough incoming kinetic energy to ionize the small amount of atmosphere present in the vacuum chamber in the path of flight to generate self-illuminated 'shooting stars'. Additionally, a thin film of Mylar $(12.7 \mu \mathrm{m})$ was placed at a known distance ahead of the target plate and created an impact flash when the incoming projectile passed through it. This flash was registered by a visible wavelength photodiode and was used to trigger the optics high-speed camera. At that same instant, a second high-speed camera (1 million frames per second) with continuous recording capabilities of up to 1.2 seconds was also triggered off the photodiode, capturing a high-speed video of the incoming micrometeoroid or space debris, the impact event itself, and the resulting energetic phenomena of plasma formation and ejecta. The second high-speed camera was also used to discern the time and distance of 'shooting star' flight between the pre-target film and the polymer target plate, and then analyzed along with the self-illuminated streak to calculate the collision velocity to an accuracy of $\pm 2 \%$. 
The optical methods of dynamic photoelasticity and caustics were used in this investigation, with caustics used to directly determine the dynamic stress intensity factor ahead of the moving crack. Optical distortions resulting from the large beam expansion $(100 \mathrm{~mm})$ were removed using the control point selection toolbox in MATLAB. This process involved taking a set of images with the highspeed camera of a known-sized grid in the field of view prior to running a series of tests in a certain configuration. In addition, a virtual grid is created in MATLAB which matches the actual grid geometry, not what appears via the camera image. Using a bicubic transformation based on control points, the code maps the possibly distorted test images to the virtual grid, thereby eliminating innate barrel or pincushion distortion. This is an important step in the analysis because without it, the caustic size and crack speeds could be misinterpreted due to optical distortion error, particularly at the edge of the field of view where this effect is exaggerated. The code can found in Appendix B.

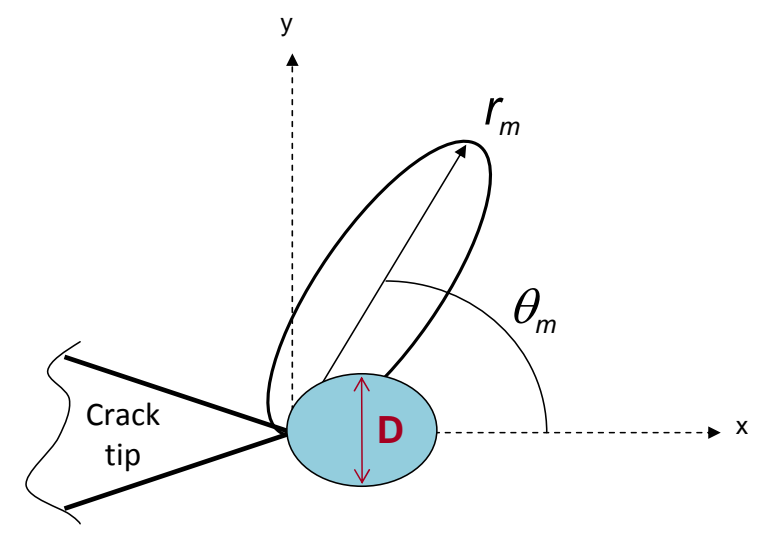

Figure 5.3.1: Schematic of information obtained by the methods of dynamic photoelasticity and caustics ahead of a moving crack tip
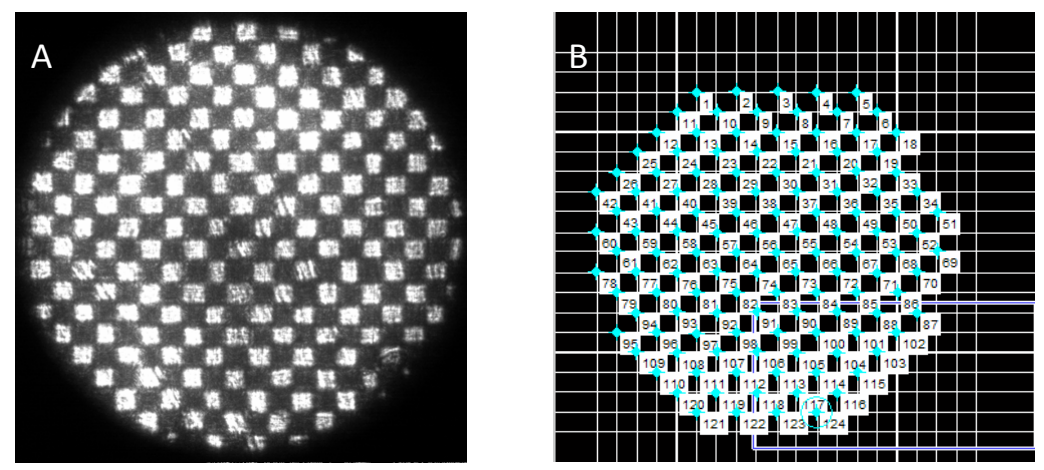

Figure 5.3.2: (A) The actual grid as seen by the high-speed camera of the $100 \mathrm{~mm}$ expanded laser beam, taken to remove optical distortions by using a (B) virtual grid created in MATLAB to map the crack growth images for analysis 


\section{Caustics}

For caustic analysis, the polymer plate is placed slightly out of the focus of the high-speed camera by a known distance, $z_{0}$. Collimated laser light is passed through the thin polymer plate specimen, and a localized stress-induced thinning effect can be seen near the crack tip (as shown in Figure 5.3.3). The uniform laser light is consequently refracted away from the crack tip creating a characteristic shadow spot, surrounded by a brighter epicycloid called a caustic [28]. The diameter of the shadow spot has been shown to relate to the dynamic stress intensity factor at that instant [43] as follows

$$
K_{I}^{d}=\zeta\left(v, E, \mu, z_{0}, C, t\right) D^{5 / 2}
$$

where $D$ is the vertical diameter of the shadow spot and $\zeta$ is a known function of crack speed, $v$, elastic properties, $E$ and $\mu$, reference plane distance, $z_{0}$, stress optical coefficient, $C$, and plate thickness, $t$.

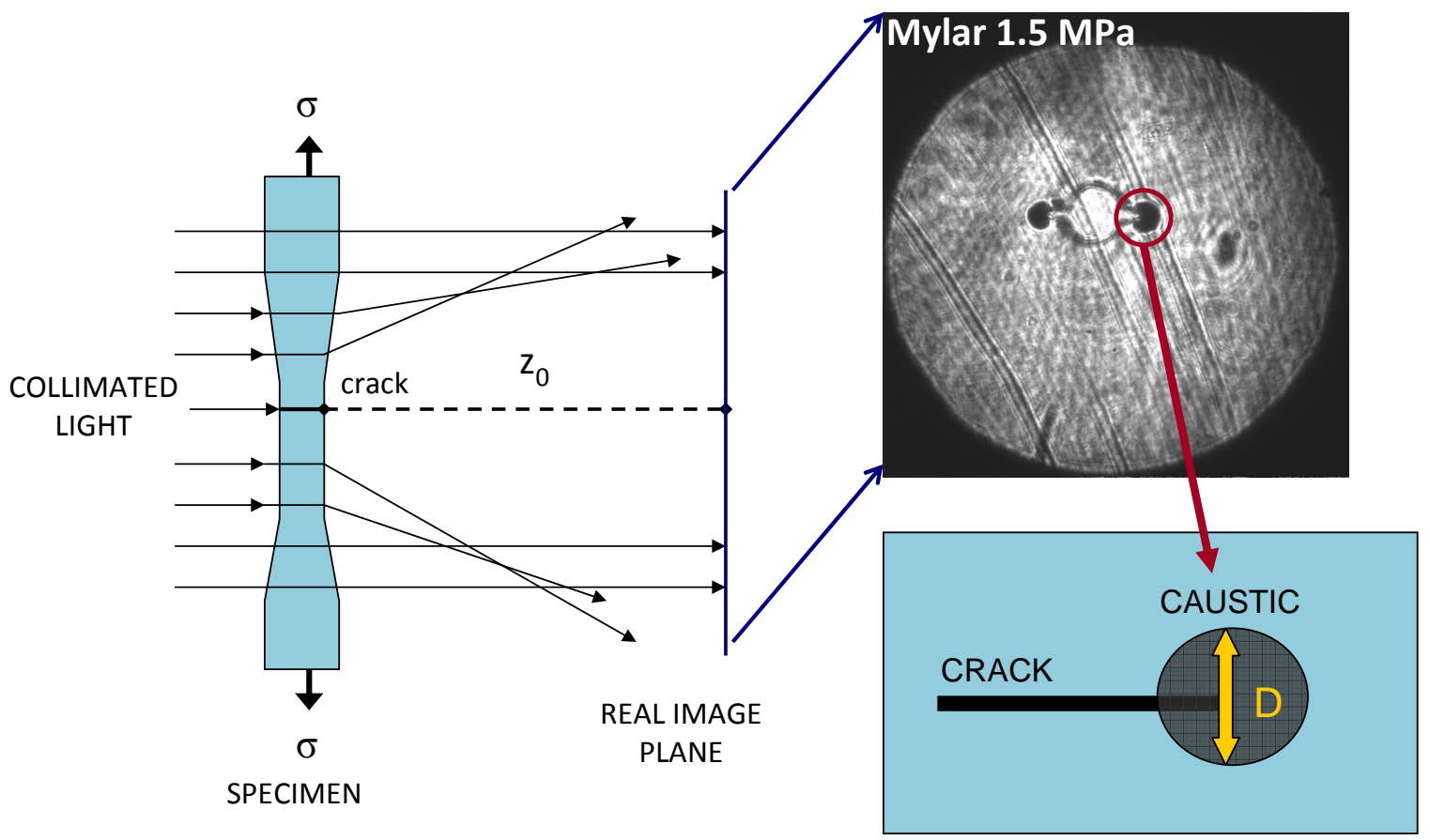

Figure 5.3.3: The method of shadow spots from caustics in transmission

In transmission, the dynamic stress intensity factor is written explicitly in terms of the diameter of the caustic, $D$, [3] or

$$
K_{I}^{d}=F(v) M D^{5 / 2}
$$


where specifically

$$
\begin{aligned}
M & =\frac{2 \sqrt{2} \pi}{3 m^{\frac{3}{2}} f^{\frac{5}{2}} c t z_{0}} \\
F & =\frac{4 \alpha_{d} \alpha_{s}-\left(1+\alpha_{s}^{2}\right)^{2}}{\left(\alpha_{d}^{2}-\alpha_{s}^{2}\right)\left(1+\alpha_{s}^{2}\right)} \\
\alpha_{d} & =1-\frac{v^{2}}{c_{d}^{2}} \\
\alpha_{s} & =1-\frac{v^{2}}{c_{s}^{2}} \\
m & =\text { scale factor } \\
f & =\text { shadow optical constant } \\
c & =\text { stress optical coefficient } \\
t & =\text { target thickness } \\
z_{0} & =\text { reference distance } \\
v & =\text { crack propagation velocity } \\
c_{d} & =\text { longitudinal wave velocity } \\
c_{t} & =\text { transverse wave velocity. }
\end{aligned}
$$

\begin{tabular}{|c|c|c|c|}
\hline & & Homalite 100 & Mylar \\
\hline Elastic Modulus (MPa) & $\mathrm{E}$ & 3860 & 2100 \\
\hline Poisson's Ratio & $\nu$ & 0.35 & 0.38 \\
\hline Density $\left(\mathrm{kg} / \mathrm{m}^{3}\right)$ & $\rho$ & 1230 & 1390 \\
\hline $\mathrm{P}$ wave speed ${ }^{\dagger}(\mathrm{m} / \mathrm{s})$ & $c_{d}$ & 2145 & 2447 \\
\hline $\mathrm{S}$ wave speed $(\mathrm{m} / \mathrm{s})$ & $c_{s}$ & 1082 & 1185 \\
\hline Rayleigh wave speed $(\mathrm{m} / \mathrm{s})$ & $c_{R}$ & 980 & 1070 \\
\hline Stress Optical Coefficient $\left(\mathrm{m}^{2} / \mathrm{N}\right)$ & $c$ & $-9.2 \times 10^{-11}$ & $-1.4 \times 10^{-10}$ \\
\hline
\end{tabular}

In the experimental configuration presented, $z_{0}$ is $1.83 \mathrm{~m}, \mathrm{~m}$ is 1.06 for a slight expansion in the laser beam over the course of the optical path, and $f$ is 3.17 , assuming optically isotropic materials. The rest of the material and optical properties are listed in Table 5.1 for both polymers considered.

Table 5.1: Elastic and optical properties of Homalite 100 and Mylar

$\dagger$ Determined by averaging 2 amplitudes from pulse-echo ultrasonic technique, $\pm 175 \mathrm{~m} / \mathrm{s}$ 
Resulting crack velocities were estimated with a secant method which evaluates the derivative of the crack length versus time history provided by the output of the high-speed camera. It should be noted that crack velocities in these tests were calculated only in the global sense dictated by the interfame time on the high-speed camera images. Thereby, the estimated crack velocities were insensitive to any transient effects at the crack tip appearing on a smaller time scale.

\section{Photoelasticity}

The photoelastic method is possible due to the stress-induced birefrigence observed in the brittle polymers, proportional to the difference in in-plane principal stresses

$$
n_{1}-n_{2}=c\left(\sigma_{1}-\sigma_{2}\right)
$$

where $n_{1}$ and $n_{2}$ are the two different indices of refraction in a stressed birefringent material, $c$ is the stress optical coefficient, and $\sigma_{1}$ and $\sigma_{2}$ are the principal stresses.

A target under stress retards the polarized light by $\delta$. The change in refractive index in the plane perpendicular to the optical axis can be written in terms of both $\delta$ and the target thickness, $t$, and the wavelength of the light $\lambda$, providing the Stress-Optic Law utilized in photoelastic analysis

$$
\sigma_{1}-\sigma_{2}=\frac{\delta \lambda}{2 \pi c h}=\frac{N \lambda}{c h}
$$

where $N$ is known as the fringe order and can be written as $N=\lambda / 2 \pi[25]$.

In this study, a circular polariscope is configured to obtain dynamic ischochromatic fringes.

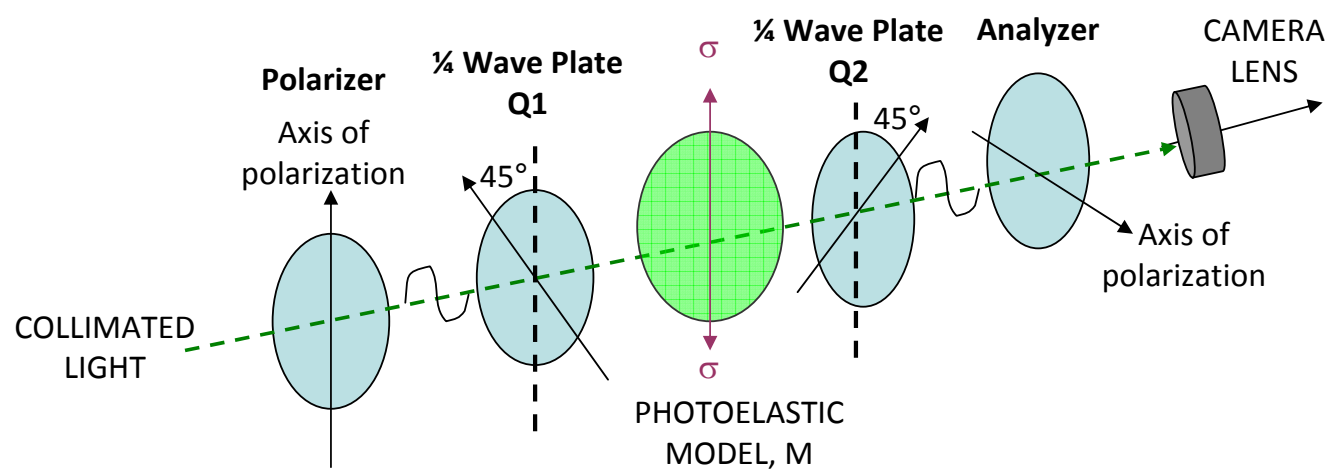

Figure 5.3.4: Schematic of circular polariscope configuration for photoelasticity 
When circularly polarized light from the first quarter wave plate, Q1, passes through the model, M, it is transmitted as two components. The planes of vibration of these components in general do not coincide with the principal stress axes. Light from the model, still circularly polarized, goes through the second quarter wave plate, Q2. The principal axis, of plate Q2 being crossed with that of plate Q1, the circular polarization is cancelled and removed by plate Q2. The light then proceeds to the analyzer as two components, and is resolved at that point into plane polarized light.

Supposing the plate is subjected to a uniform stress field, superposing Irwin's solution for stresses near a crack tip [23] and neglecting higher order terms, the stress intensity factor from this method can be expressed as

$$
K_{I}^{d}=\frac{N f \sqrt{2 \pi r_{m}}}{t \sin \theta_{m}}\left[1+\left(\frac{2}{3 \tan \theta_{m}}\right)^{2}\right]^{0.5}\left(1+\frac{2 \tan \left(\frac{3 \theta_{m}}{2}\right)}{3 \tan \theta_{m}}\right)
$$

where the quantities $N, r_{m}$ and $\theta_{m}$ are determined from a single isochromatic loop as shown in Figure 5.3.1 [18].

The photoelastic method was not pursued for dynamic stress intensity factor calculations due to the lack of usable frames and fringes from the high-speed camera images. Additionally, energetic phenomena such as ejecta and debris clouds obstructed some of the crack tip images. In all of Homalite 100 cases, the fracture process occurred after impact stress waves had reflected with the boundaries and interfered with the isochromatic fringes at the crack tip.

\subsection{Results \& Conclusions}

Both polymers sustain globally subsonic crack tip speeds of roughly $0.2-0.47 c_{R}(180-450 \mathrm{~m} / \mathrm{s})$, the Rayleigh wave speed, or the slowest solid wave speed which traverses across the surface of the material. Upon impact Homalite 100 exhibited a distinctive oscillation in the crack path appearance, and took slightly longer (on average $20 \mu \mathrm{s}$ ) than Mylar to completely propagate across the diameter of the plate. Cracks in Homalite 100 featured shadow spots whose size varied with time more noticeably than in Mylar, indicating more frequent variations in stress intensity factor and crack tip speeds. Conversely, Mylar exhibited a flat crack path appearance, slightly faster crack growth speeds, with crack tips closely following the shear wave. Regardless, both Mylar and Homalite 100 seem to demonstrate behavior which was consistent with crack path selection morphologies expected under local mode-I conditions. For perspective, shadow spots and caustics surrounding the crack tip propagation are shown in Figure 5.4.4 following an impact event on Mylar. 

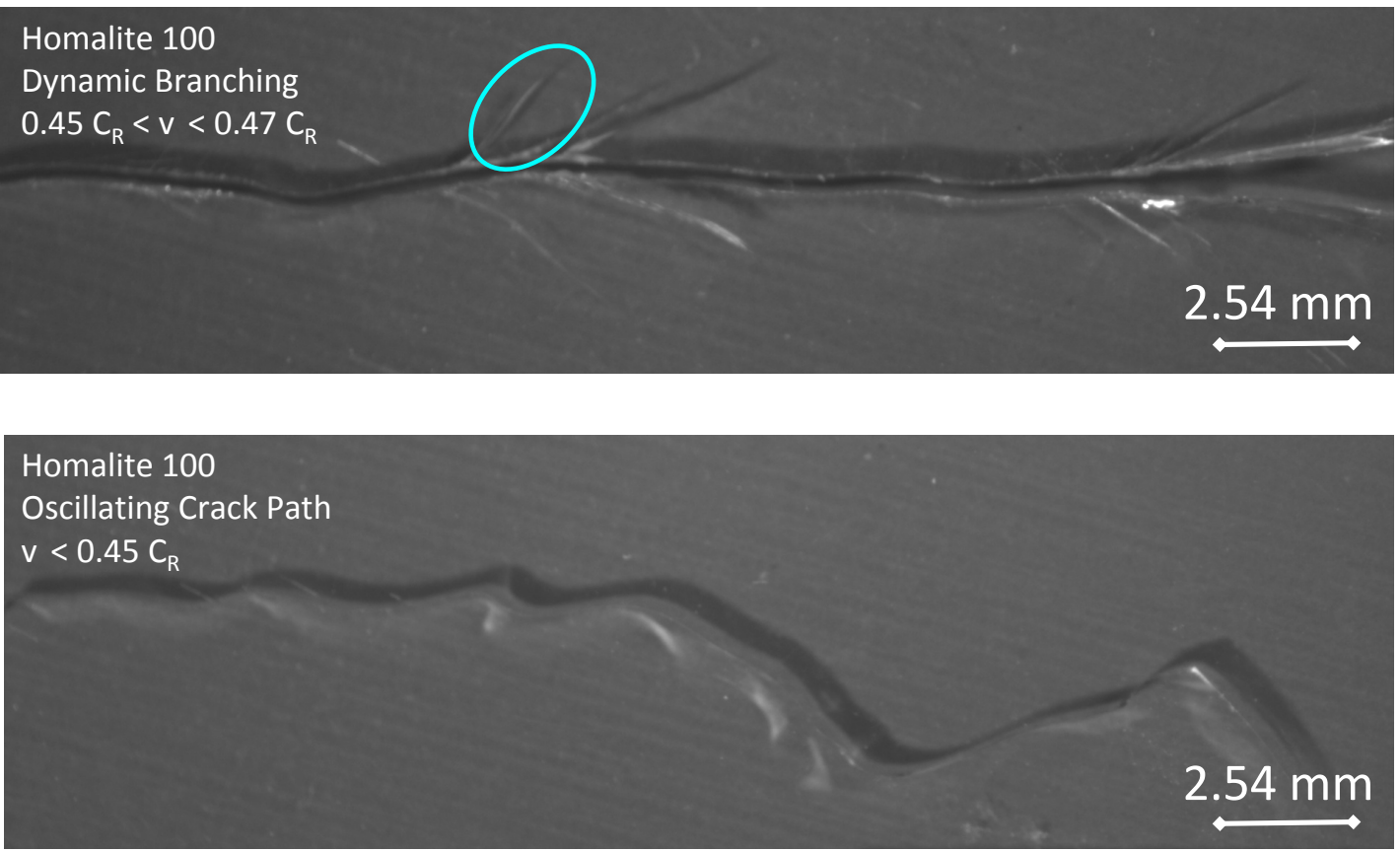

Figure 5.4.1: Homalite 100 resulting crack path appearance. Nearly all cases exhibited an oscillating crack path (Bottom), except in the highest velocity crack speed cases where the Rayleigh wave speed was greater than $0.45 c_{R}$, and the crack path would branch (Top). Average branching angle was approximately $29^{\circ}$

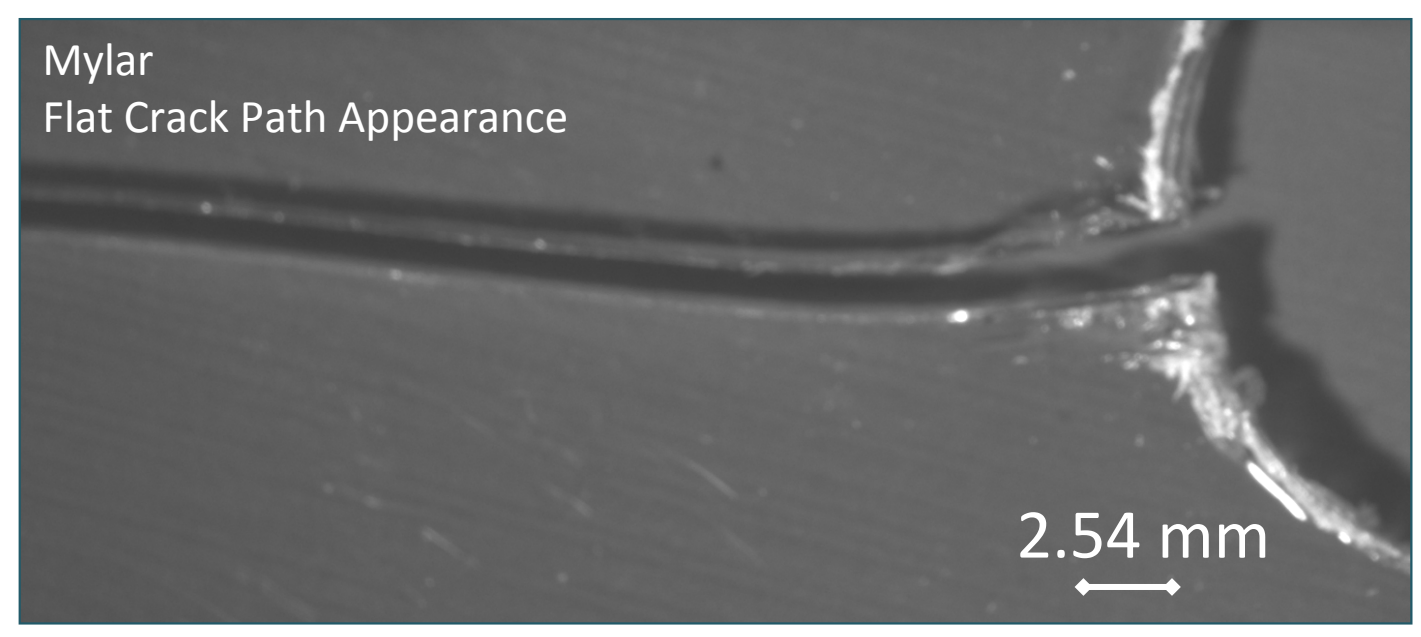

Figure 5.4.2: Mylar resulting flat crack path appearance 


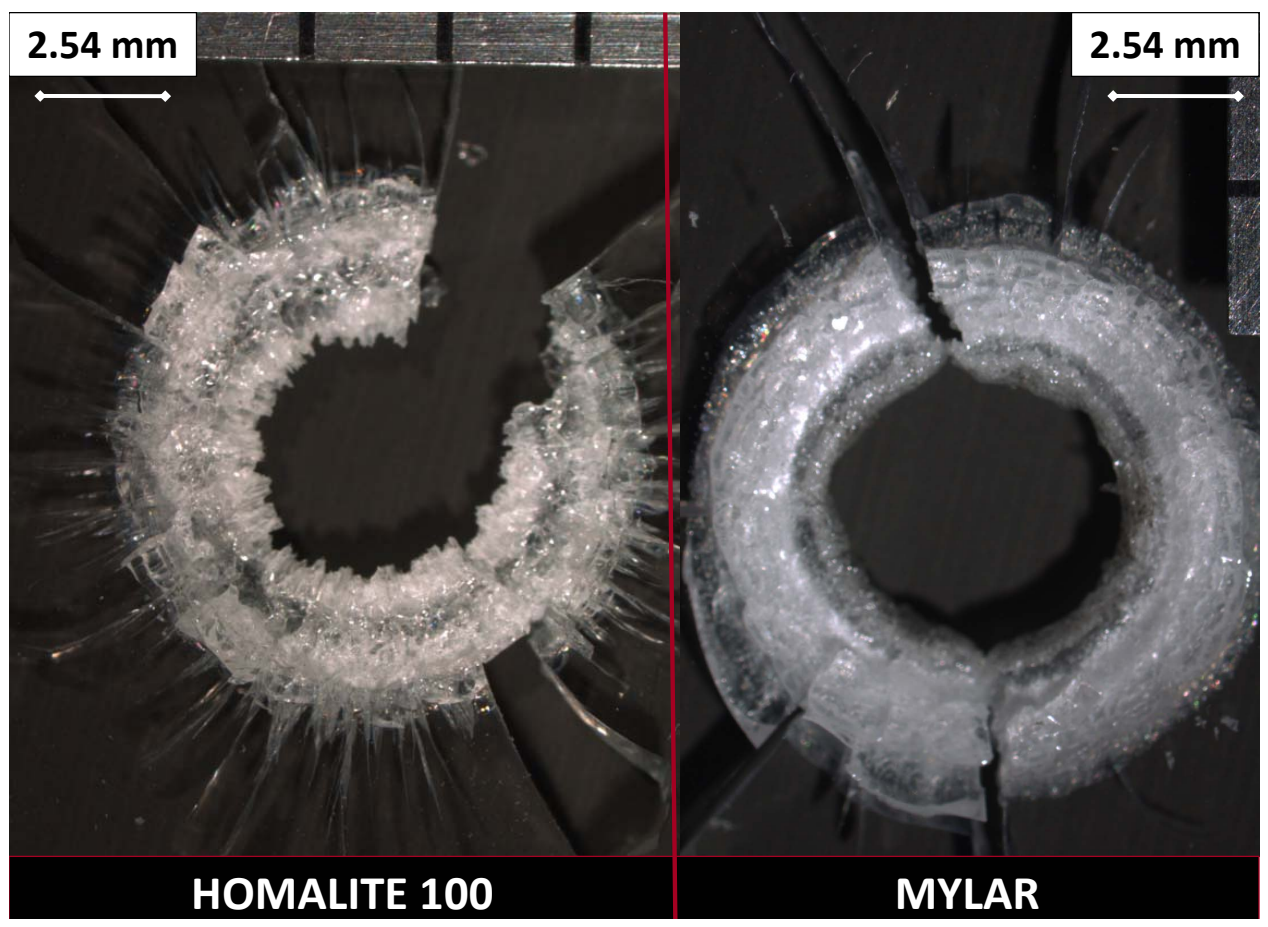

Figure 5.4.3: Microscopy images of polymer hypervelocity impact sites (Left) Homalite 100, (Right) Mylar

Figure 5.4.3 shows the impact sites of Homalite 100 and Mylar compared side-by-side. Each was hit with a nylon 6-6 right cylindrical slug $1.8 \mathrm{~mm}$ in length and diameter at approximately $5 \mathrm{~km} / \mathrm{s}$. Notice that the Homalite 100 site has extensive dynamic branching, whereas the Mylar site has a glassy-like appearance of melt fronts. One of the reasons for this difference could possibly be due to the fact that the thermal conductivity of Mylar is about 30\% lower than Homalite 100. Additionally, the size of the impact holes in both materials are greater than the diameter of the hypervelocity projectile.

In conclusively validating the assertion that mode-I classical dynamic fracture mechanics are a valid approach to describing hypervelocity impact dynamic fracture behavior of brittle polymers, the measured crack tip speed histories were obtained from the tests, each involving 4 to 8 usable highspeed camera frames of the fracture process. The shadow spot diameter from each frame, together with the calculated speeds were used to determine the critical dynamic stress intensity factors and energy release rates at each time instant. While over 100 experiments were performed in this process, only results from 40 tests where cracks were growing, were used for the data, so that the values of the dynamic energy release rate reported here are by definition critical, $G^{d}=G_{I C}^{d}$. The values 
of $G_{I C}^{d}$, averaged per test are plotted against corresponding averaged crack tip speeds for both Homalite 100 and Mylar in Figures 5.4.11 and 5.4.10, respectively. The data exhibits the $\Gamma$-shaped trend noted in literature for typical mode-I results [7]. In addition, Figure 5.4.11 also includes a solid curve corresponding to $G_{I C}^{d}$ versus $v$ data obtained from multiple experiments involving significantly lower loading rates and conventional, purely in-plane loading configurations [13].
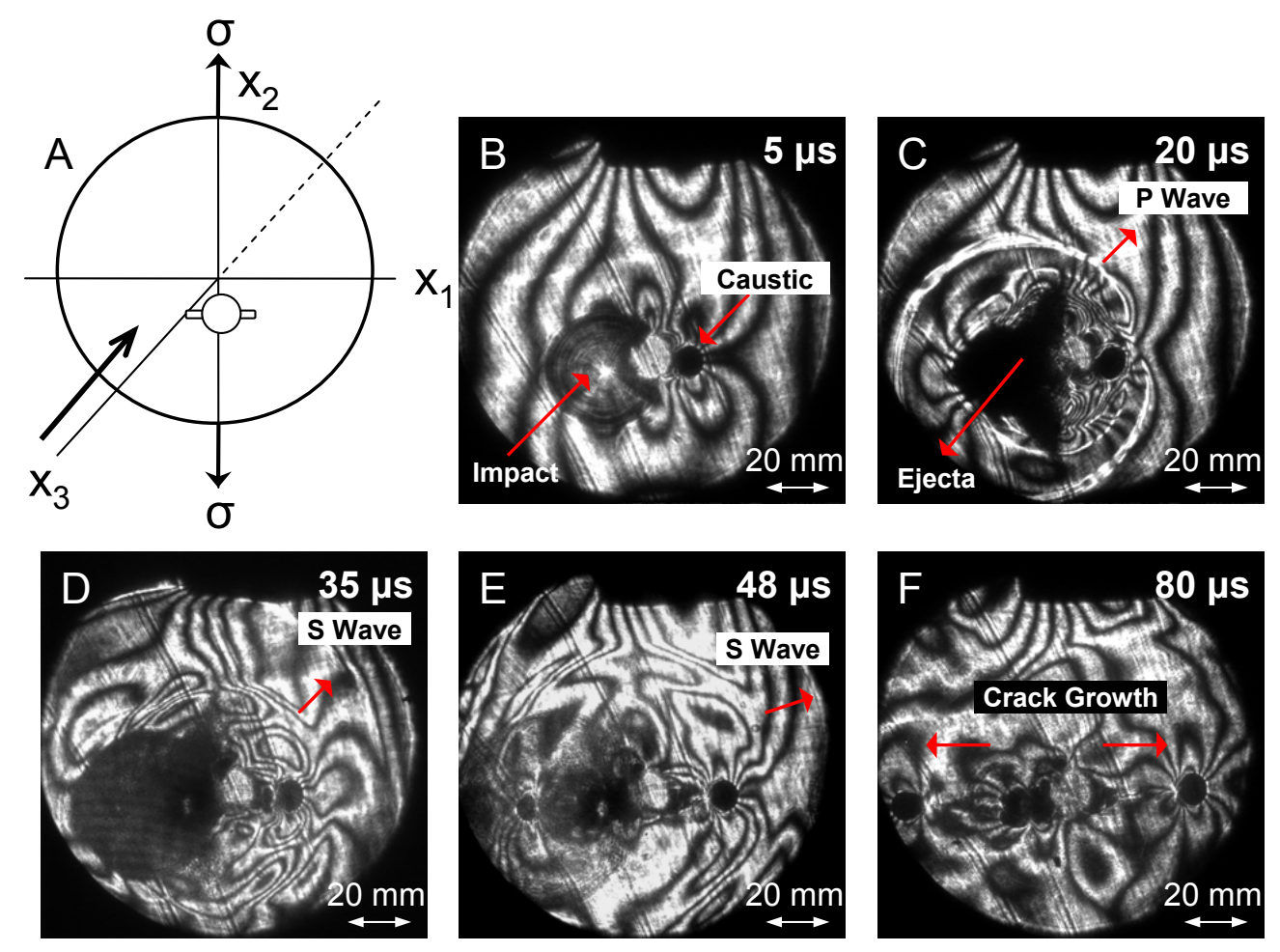

Figure 5.4.4: High-speed photography is used to capture the isochromatic fringe pattern, caustics and shadow spots generated during crack growth resulting from a micrometeoroid or space debris hypervelocity impact at $4.5 \mathrm{~km} / \mathrm{s}(10000 \mathrm{mph})$ with Mylar plate, $150 \mathrm{~mm}$ diameter and $1.6 \mathrm{~mm}$ thick, held in tension at $3.75 \mathrm{MPa}$. (A) Configuration of the pre-cracked polymer plate, in plane $x_{1}$ and $x_{2}$ prior to hypervelocity impact loading from the $x_{3}$, out-of-plane, direction. (B) Upon impact, the shadow spot shown has not yet felt the impact shock. (C) The fastest stress wave, the longitudinal wave, travels radially outward from the impact site at $2447 \mathrm{~km} / \mathrm{s}$ and disturbs the caustic, but there is no crack growth. Ejecta is thrown from the plate and clouds the field of view. (D) The crack begins to grow, ejecta is starting to disperse and the shear wave is seen moving radially outwards from the impact site at $1185 \mathrm{~m} / \mathrm{s}$. (E) The crack appears to grow just behind the shear wave. (F) After $80 \mu \mathrm{s}$ the polymer plate has almost completely failed; cracks speeds averaged $360 \mathrm{~m} / \mathrm{s}(800$ $\mathrm{mph})$ 

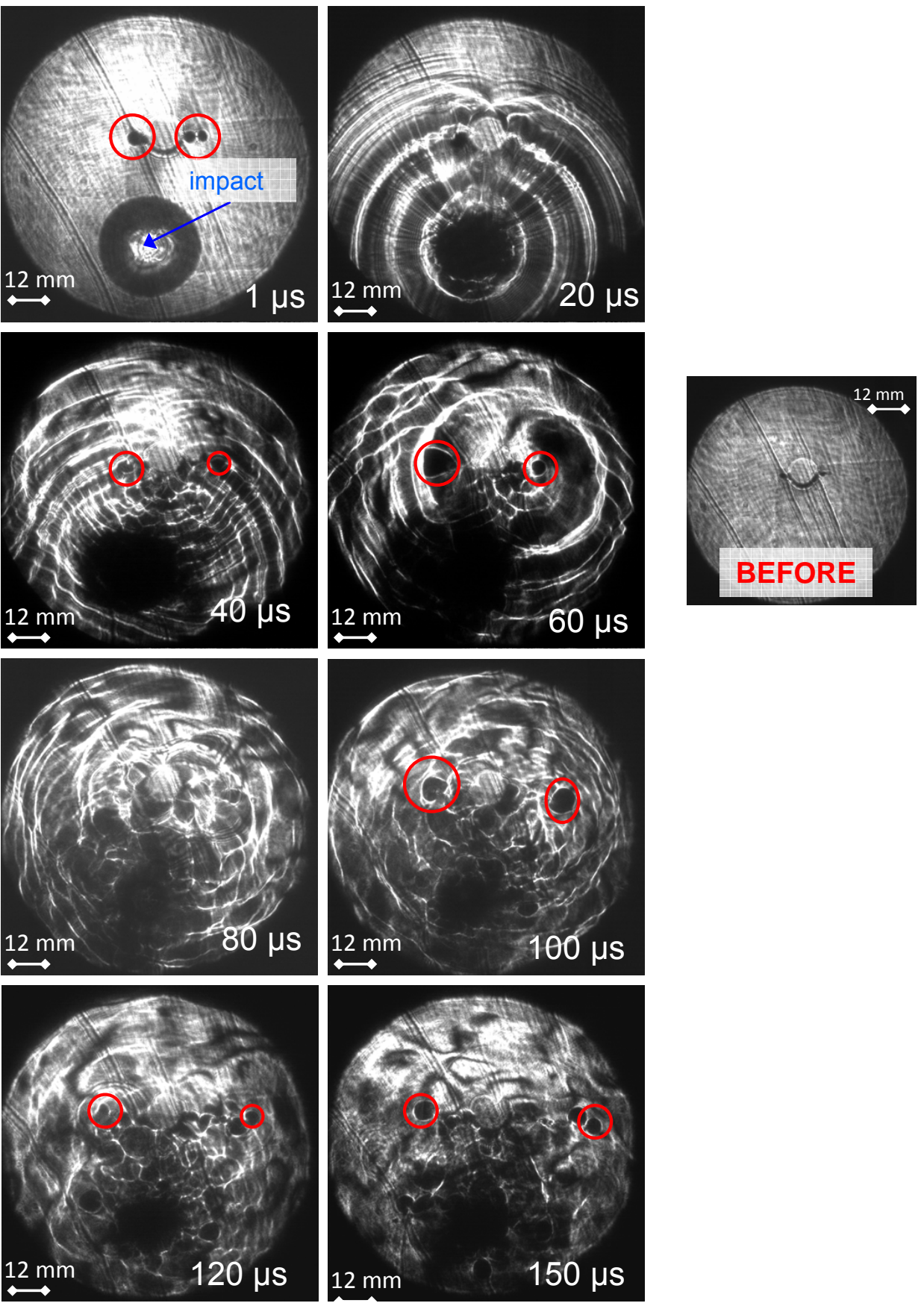

Figure 5.4.5: High-speed photography is used to capture the isochromatic fringe pattern, caustics and shadow spots generated during crack growth resulting from a nylon cylindrical slug, $1.8 \mathrm{~mm}$ in length and diameter, impacting a Homalite 100 plate, $150 \mathrm{~mm}$ diameter and $3.2 \mathrm{~mm}$ thick, which was pre-cracked and held in $1 \mathrm{MPa}$ tension, at $5.5 \mathrm{~km} / \mathrm{s}(12300 \mathrm{mph})$. Caustics and shadow spots surrounding the growing crack tip are circled in red. Resulting crack growth arrested and crack velocity averaged approximately $100 \mathrm{~m} / \mathrm{s}(220 \mathrm{mph})$ 

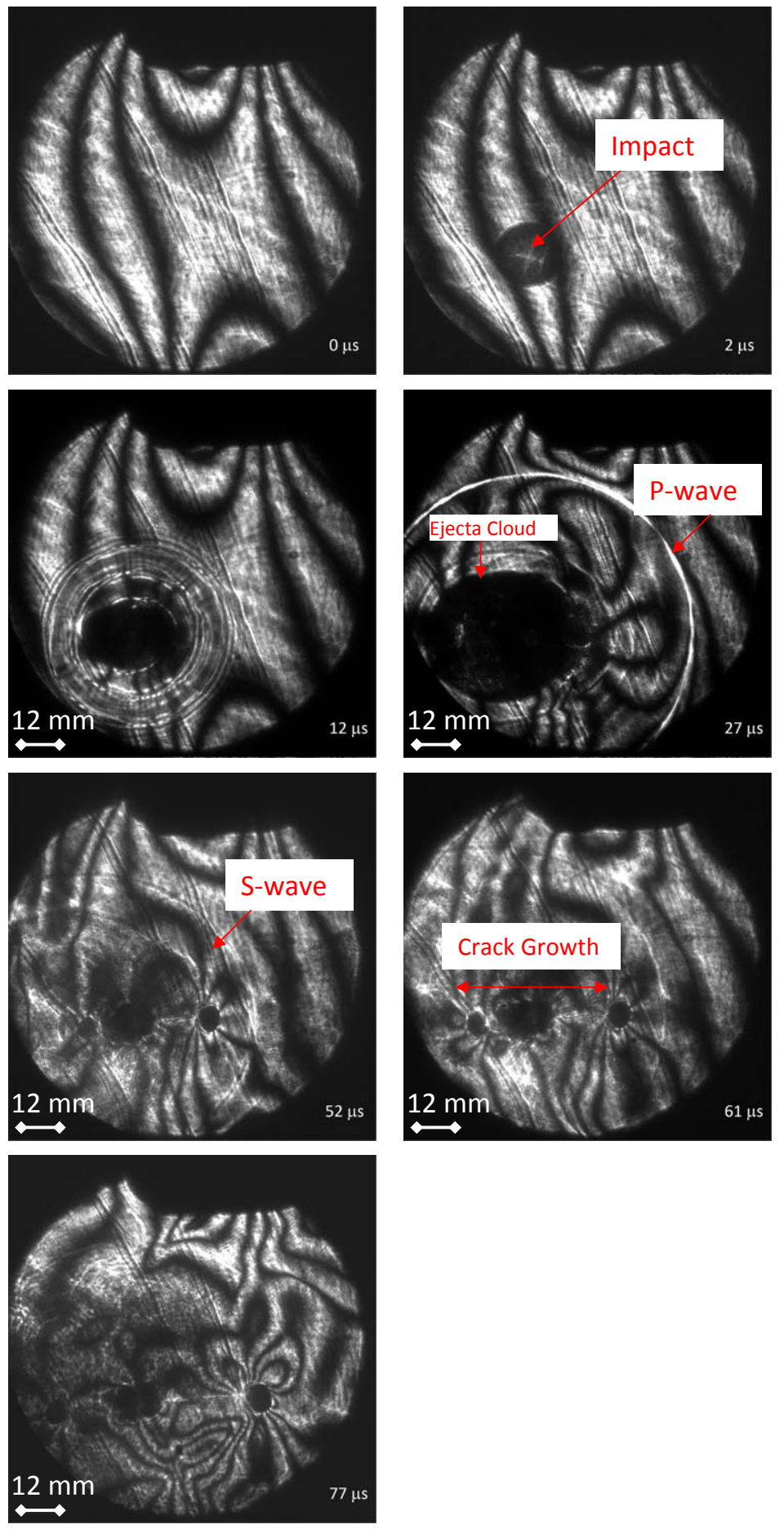

Figure 5.4.6: High-speed photography is used to capture the isochromatic fringe pattern, caustics and shadow spots generated during crack growth resulting from a nylon cylindrical slug, $1.8 \mathrm{~mm}$ in length and diameter, impacting a Mylar plate, $150 \mathrm{~mm}$ diameter and $1.6 \mathrm{~mm}$ thick, with no initial notch or pre-crack held at $3.7 \mathrm{MPa}$ in tension, at $4.3 \mathrm{~km} / \mathrm{s}(9600 \mathrm{mph})$. Resulting crack growth velocity averaged approximately $350 \mathrm{~m} / \mathrm{s}$ (780 $\mathrm{mph})$ 


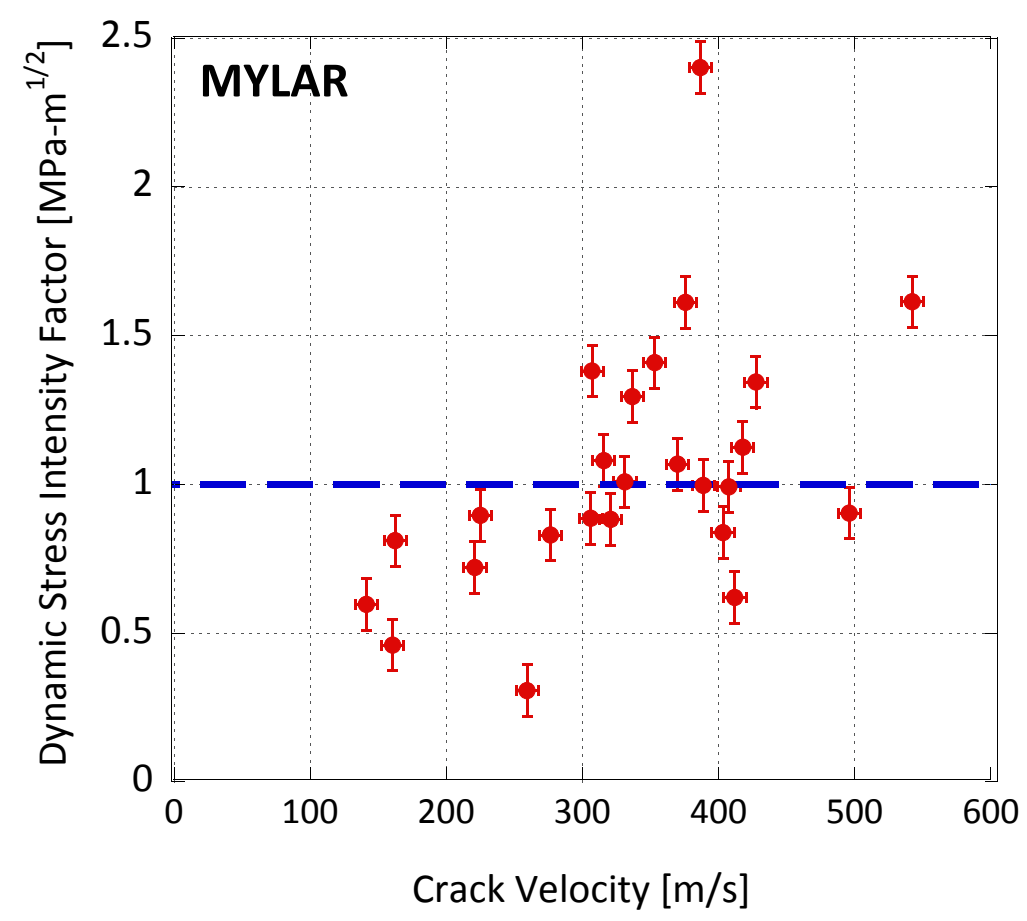

Figure 5.4.7: Dynamic stress intensity factor (mode-I) at the crack tip as a function of crack speeds resulting from hypervelocity impact induced fracture on Mylar thin plates. The quasi-static fracture toughness value is labeled by a dashed line across the horizontal axis. Unlike Homalie 100, the Mylar data points are not averaged per entire test since the mode-I fracture trend was clear before averaging. Each data point corresponds to one high-speed photograph from a test 


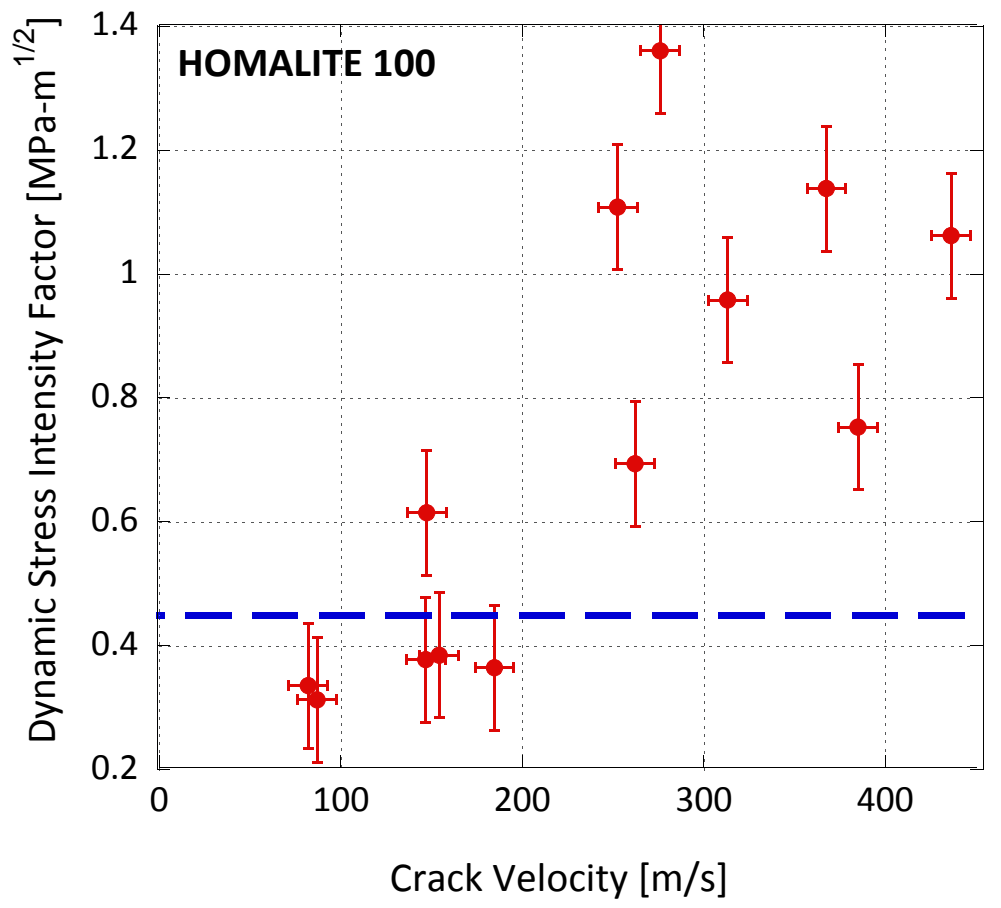

Figure 5.4.8: Averaged dynamic stress intensity factor (mode-I) at the crack tip as a function of crack speeds resulting from hypervelocity impact induced fracture on Homalite 100 thin plates. The quasi-static fracture toughness value is labeled by a dashed line across the horizontal axis 


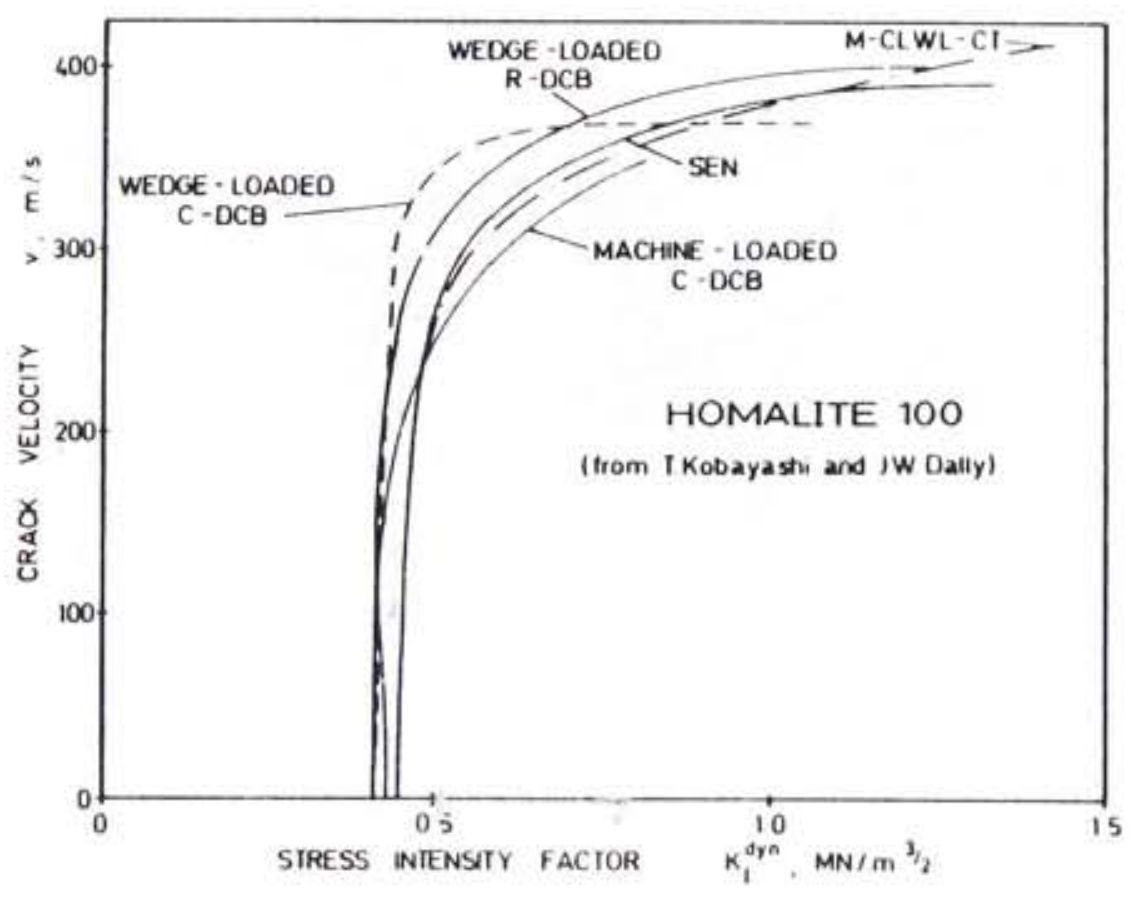

Figure 5.4.9: Dynamic stress intensity factor versus crack tip speeds in Homalite 100 for various double cantilever beam (DCB) and single edge notch (SEN) combinations of classical mode-I configurations from Kobayashi and Dally [3] 


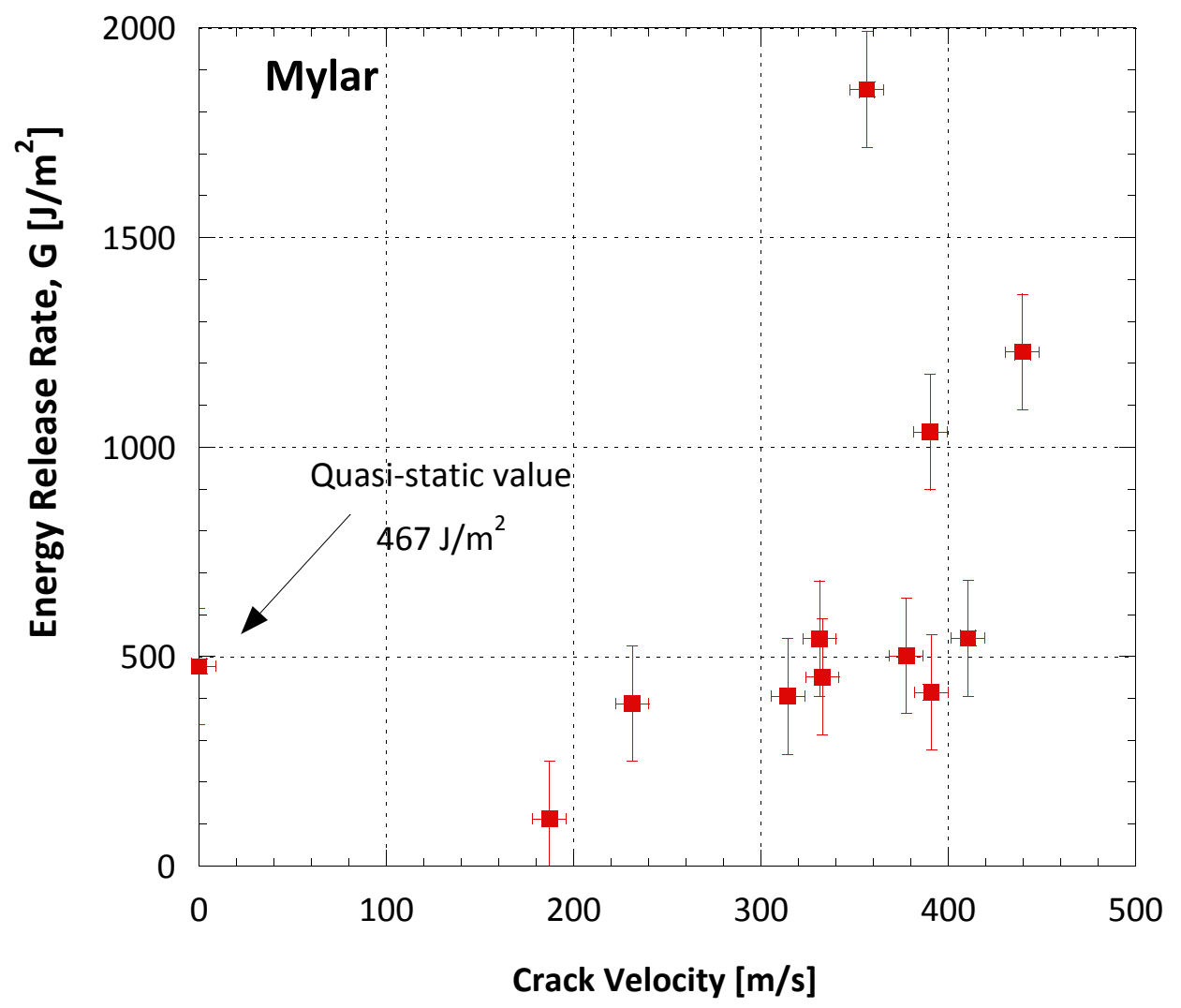

Figure 5.4.10: Averaged dynamic energy release rates at the crack tip as a function of crack speeds resulting from hypervelocity impact induced fracture on Mylar thin plates. The quasi-static energy release rate value $\left(v=0^{+}\right)$is labeled on the vertical axis 


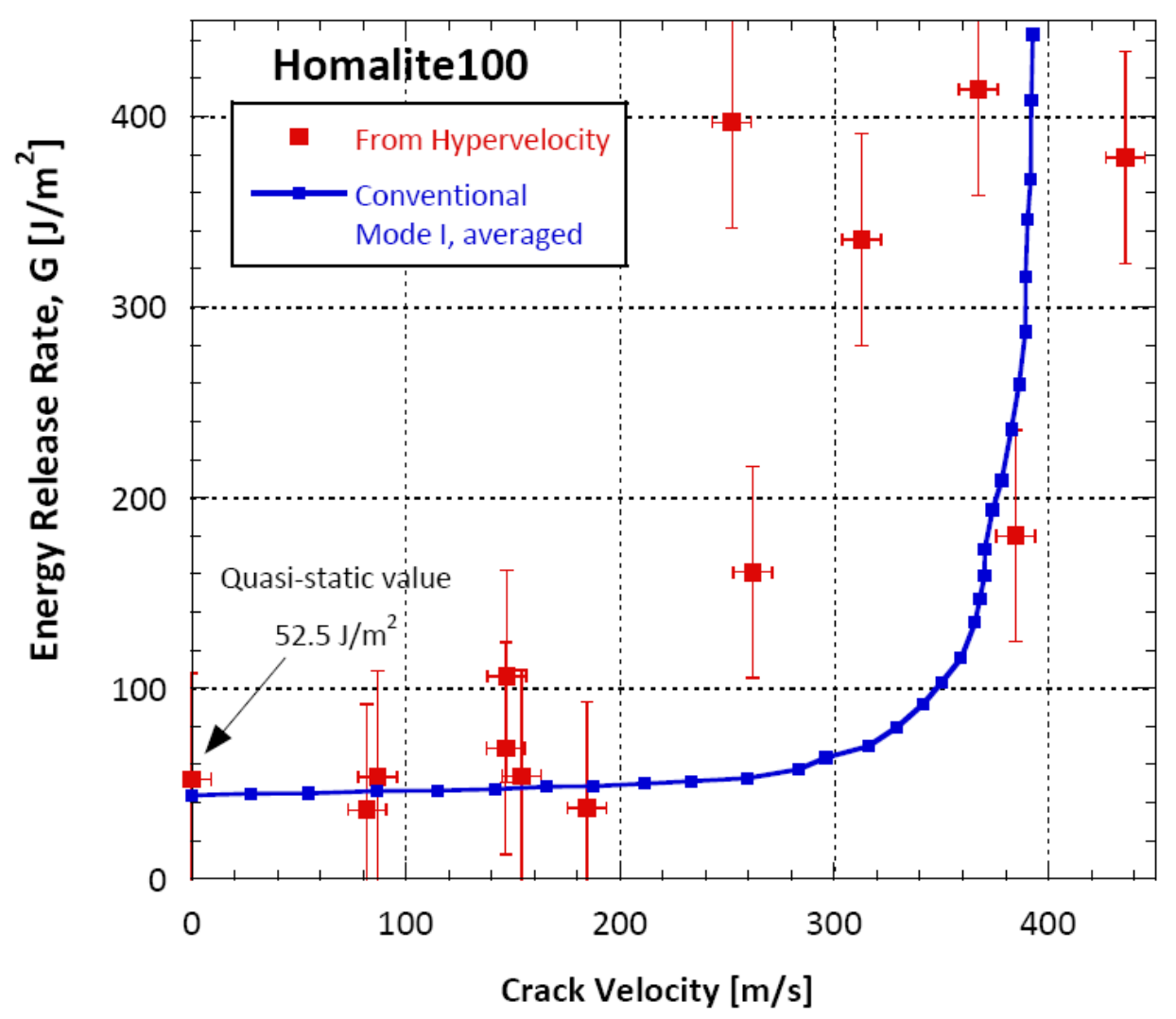

Figure 5.4.11: Averaged dynamic energy release rates at the crack tip as a function of crack speeds resulting from hypervelocity impact induced fracture on Homalite 100 thin plates. The quasi-static energy release rate value $\left(v=0^{+}\right)$is labeled on the vertical axis. The solid thin line illustrates averaged values from numerous conventional, purely mode-I or crack-opening configurations, with in-plane loading conditions

A couple of significant observations can be made on the basis of the results displayed in Figures 5.4.10 and 5.4.11. In both material systems tested, the critical energy provided to the crack tip for growth appears to have a well-defined dependence on crack tip speed. This dependence exhibits a drastic increase in $G_{I C}^{d}$ at about $0.45 c_{R}$. For Homalite 100, this value is close to the crack branching speed [27]. Perhaps the most significant observation is that the solid line obtained from averaging multiple in-plane loading configurations at much lower structural loading rates lies within the current experimental results that have been obtained under loading conditions involving extremely energetic out-of-plane hypervelocity impacts. The consequence of these observations is that classical mode-I dynamic fracture mechanics criteria still remain valid during hypervelocity impact and as a result, dynamic fracture methodologies can still be used for the safe design of thin shell space structures. 
Table 5.2: Results from optical investigations of hypervelocity impact dynamic fracture of brittle polymers

\begin{tabular}{lcc}
\hline & Homalite 100 & Mylar \\
\hline Average crack tip speed $[\mathrm{m} / \mathrm{s}]$ & 230 & 330 \\
Quasi-Static Fracture Toughness ${ }^{\ddagger}[\mathrm{MPa}-\sqrt{\mathrm{m}}]$ & 0.45 & 1.0 \\
Averaged Dynamic Stress Intensity $[\mathrm{MPa}-\sqrt{\mathrm{m}}]$ & 0.73 & 1.0 \\
Quasi-Static Energy Release Rate $\left[\mathrm{J} / \mathrm{m}^{2}\right]$ & 52.5 & 467 \\
Averaged Dynamic Energy Release Rate $\left[\mathrm{J} / \mathrm{m}^{2}\right]$ & 208 & 690 \\
Crack Path Appearance & Oscillating & Flat \\
\hline$\dagger$ Determined by averaging 2 amplitudes from pulse-echo ultrasonic technique, $\pm 175 \mathrm{~m} / \mathrm{s}$ \\
$\ddagger$ From literature, Mylar from Shockey $1981[47]$, Homalite from Mall and Kobayashi $1978[7]$
\end{tabular}

The aforementioned conclusion can be rationalized by emphasizing that in the specific hypervelocity impact investigations examined, the projectile (a.k.a. micrometeoroid or space debris) completely inserts itself in the thin polymer target with speeds that are up to 7 times greater than the Rayleigh surface wave speeds of the target. As a result, the insertion process is completed on a time period which is of the order of a third of a microsecond and involves almost instantaneous release of extreme amounts of surface energy, similar to an embedded localized explosion. Due to the small thickness of the target, and the extremely fast insertion process, much of this energy is available for in-plane stress generation and in-plane crack driving force creation. Moreover, the entire crack growth process following projectile insertion takes place well before multiple reflected waves from the boundaries have the time to fully develop an out-of-plane flexural (bending) mode of plate deformation. 


\section{Appendix A}

\section{Additional Statistical Analysis}

\section{A.1 F-Test for Equality of Variances}

When comparing the Mylar flash method with the streak method, the standard deviations can be shown to be not significantly different using an F-test with a $10 \%$ level of significance. In this situation the null hypothesis, $H_{0}$, is that the standard deviations between the two velocimetry methodologies are the same, and the alternate hypothesis, $H_{a}$, is that the standard deviations between the two velocimetry methodologies are not the same.

The F-value for velocimetry measurement 1 (Mylar flash) and velocity measurement 2 (streak or streak hybrid) is calculated as:

$$
\text { F-value }=\frac{s_{2}^{2}}{s_{1}^{2}}
$$

where $s$ is the standard deviation for the specific sample. This F-value would have $n_{2}-1$ and $n_{1}-1$ degrees of freedom, respectively [36].

For the low velocity regime, the standard deviation of the hybrid streak method was $180.2 \mathrm{~m} / \mathrm{s}$, and the standard deviation of the trigger flash method was $169.8 \mathrm{~m} / \mathrm{s}$. Each method had 16 degrees of freedom and gave a calculated F-value of 1.13. Using F-Tables for this combination of degrees of freedom and a $10 \%$ level of significance provides an F-value of $2.333 .{ }^{1}$ Since the F-value from the tables is larger than the calculated value from the experiments, the null hypothesis cannot be rejected. Therefore, it cannot be concluded that the standard deviations are statistically different.

\footnotetext{
${ }^{1}$ Note that this is a two-sided alternate hypothesis, so this is a $5 \%$ upper tailed F-value.
} 
In the high velocity regime, the standard deviation for the streak method was $1.17 \mathrm{~km} / \mathrm{s}$ and the standard deviation for the trigger flash method was $1.09 \mathrm{~km} / \mathrm{s}$. Each method had 17 degrees of freedom, corresponding to an F-value of 1.15. Using F-Tables for this combination of degrees of freedom and a $10 \%$ level of significance gives an F-value of 2.272 [36]. Like the low velocity regime, since the table value is larger than the calculated value from the experiments, the null hypothesis cannot be rejected and the standard deviation of the two methods cannot be assumed to be significantly different.

\section{A.2 Anderson-Darling Test for Normality}

Given a the null hypothesis, $H_{0}$, that the velocimetry data is normally distributed, and the alternate

hypothesis, $H_{a}$, the velocimetry data is not normally distributed, the Anderson-Darling test statistic is defined as follows

$$
A^{2}=-n-S
$$

where $n$ is the sample size and $S$ equals

$$
S=\sum_{i=1}^{n} \frac{2 i-1}{n}\left[\ln F\left(Y_{i}\right)+\ln \left(1-F\left(Y_{n+1-i}\right)\right)\right]
$$

and $Y_{i}$ is the ordered velocity data points from a specificed method, evaluated by $F$, the normal cumulative distribution function.

The null hypothesis is rejected if $A$, the test statistic, is greater than a critical value to some specified level of significance [21].

\section{A.3 Levene Test for Equality of Variances}

Given a the null hypothesis, $H_{0}$, that the variances of each velocity method are equal $\left(\sigma_{i}=\sigma_{j}\right)$, and the alternate hypothesis, $H_{a}$, that the variances are not equal $\left(\sigma_{i} \neq \sigma_{j}\right)$, the Levene test statistic is defined as follows

$$
W=\frac{(n-k) \sum_{i=1}^{k} n_{i}\left(\bar{Z}_{i .}-\bar{Z}_{. .}\right)^{2}}{(k-1) \sum_{i=1}^{k} \sum_{j=1}^{n_{i}}\left(Z_{i j}-\bar{Z}_{i .}\right)^{2}}
$$

where $n$ is the sample size, $n_{i}$ is the sample size of a subgroup (in this case the subgroup consists of $i$ and $j$ since 2 methods of measuring velocity were investigated), $k$ is a subgroup count, $Y$ is the 
actual variable value, and $Z$ is defined by

$$
Z_{i j}=\left|Y_{i j}-\bar{Y}_{i}\right|
$$

where $\bar{Y}_{i}$ is a mean of the subgroup.

The null hypothesis is rejected if $W$, the test statistic, is

$$
W>F(\alpha, k-1, n-1)
$$

or greater than the $\mathrm{F}$ distribution described earlier evalued for some level of significance, $\alpha$, and some degrees of freedom defined by $k-1$ and $n-1$.

Whereas the F-test is dependent on the assumption of normality, the Levene test is not. The Levene test can be used with any continuous distribution and is considered robust to deviations from normality [21].

\section{A.4 Velocity Distributions}

Other distributional fits for velocimetry statistical analysis:

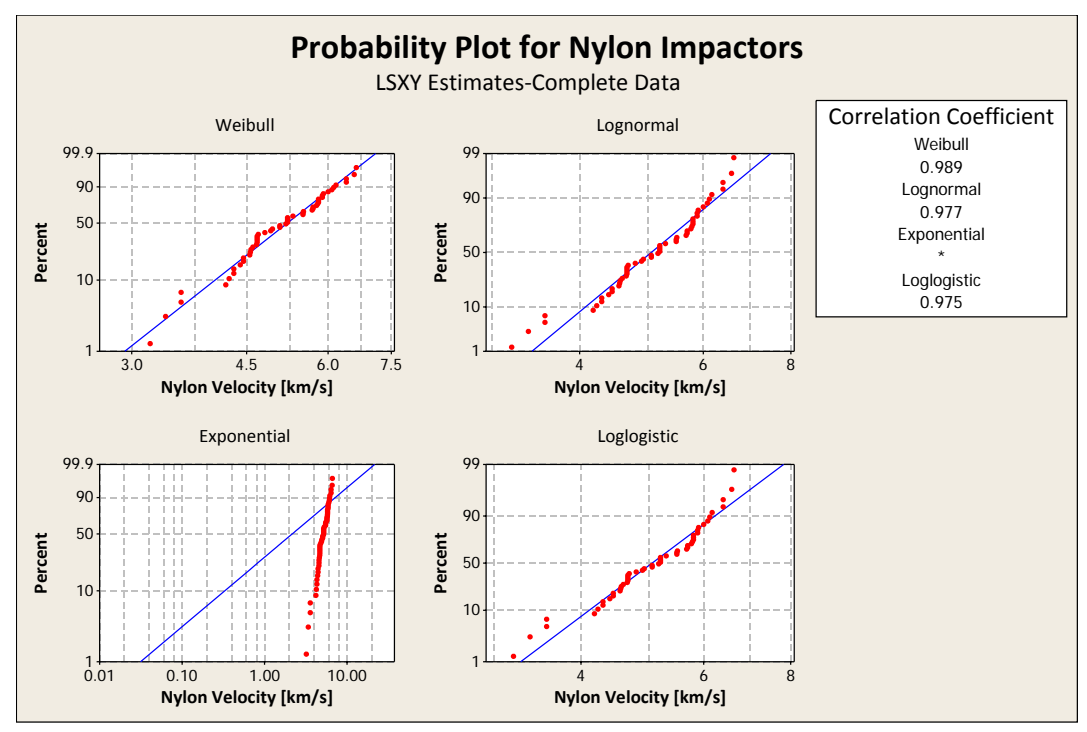

Figure A.4.1: Additional distributions fit to nylon 6-6 cylindrical slug, $1.8 \mathrm{~mm}$ length and diameter, hypervelocimetry data 


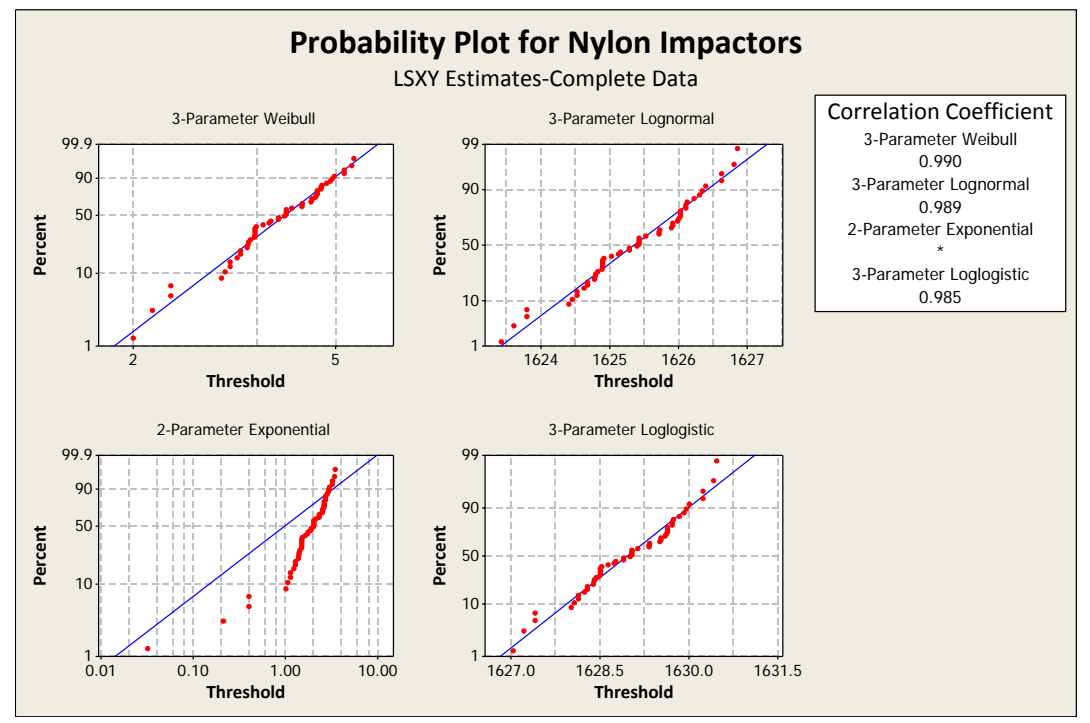

Figure A.4.2: Additional distributions fit to nylon 6-6 cylindrical slug, $1.8 \mathrm{~mm}$ length and diameter, hypervelocimetry data

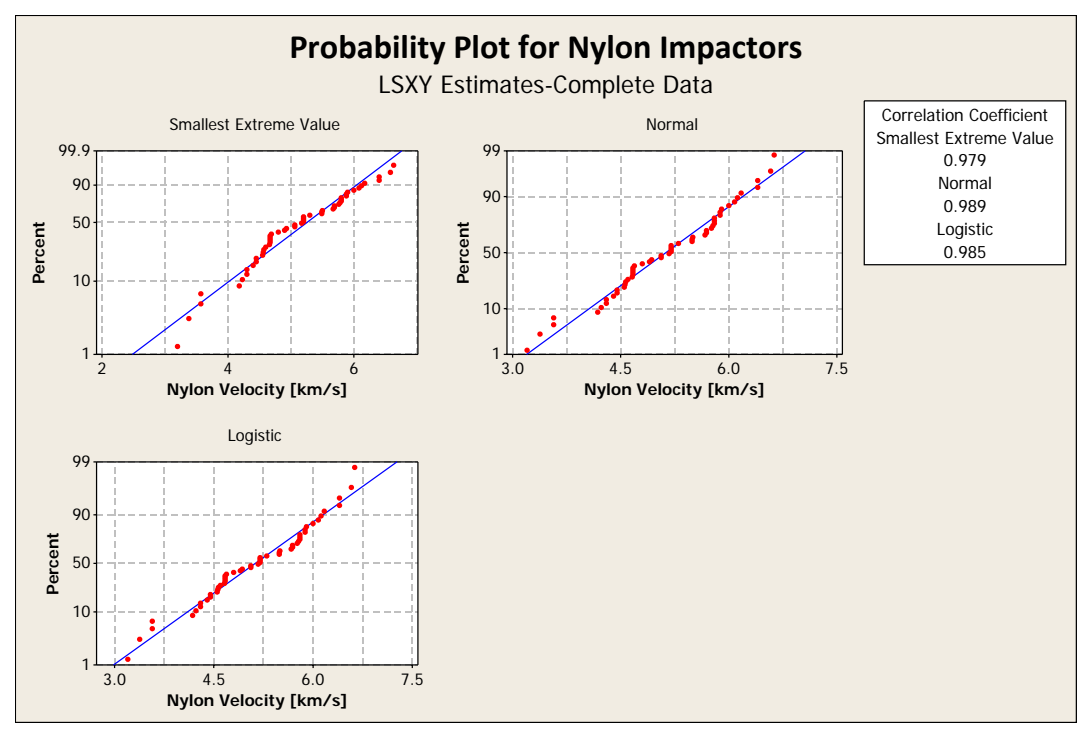

Figure A.4.3: Additional distributions fit to nylon 6-6 cylindrical slug, $1.8 \mathrm{~mm}$ length and diameter, hypervelocimetry data 


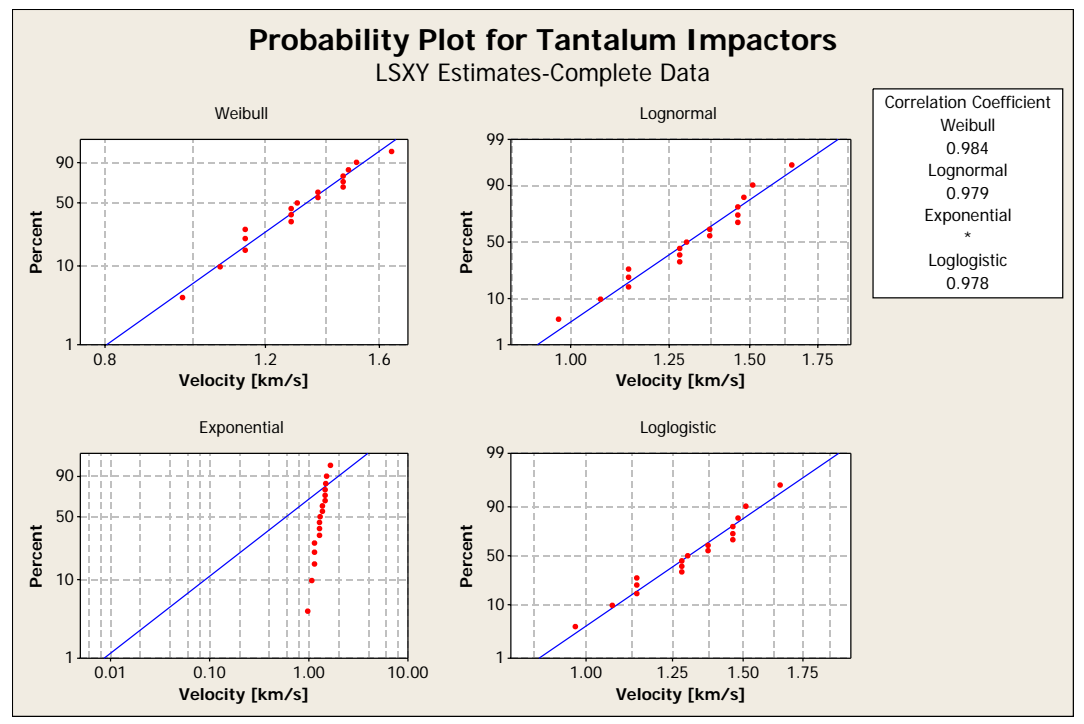

Figure A.4.4: Additional distributions fit to tantalum spheres, $1.8 \mathrm{~mm}$ diameter, hypervelocimetry data

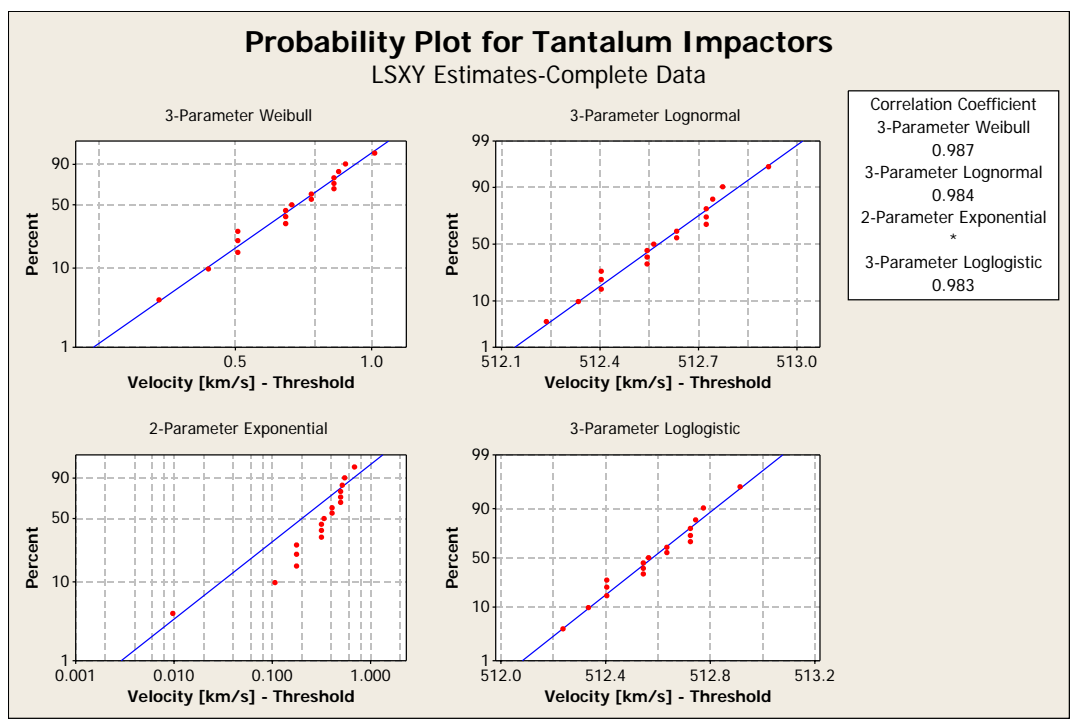

Figure A.4.5: Additional distributions fit to tantalum spheres, $1.8 \mathrm{~mm}$ diameter, hypervelocimetry data 


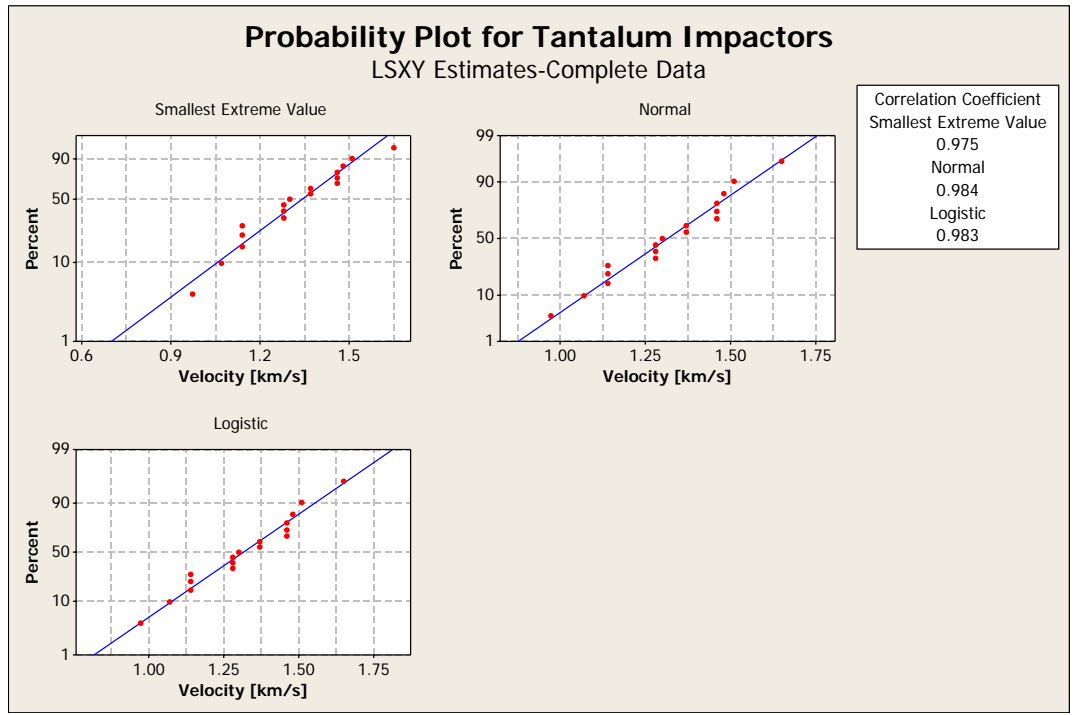

Figure A.4.6: Additional distributions fit to tantalum spheres, $1.8 \mathrm{~mm}$ diameter, hypervelocimetry data

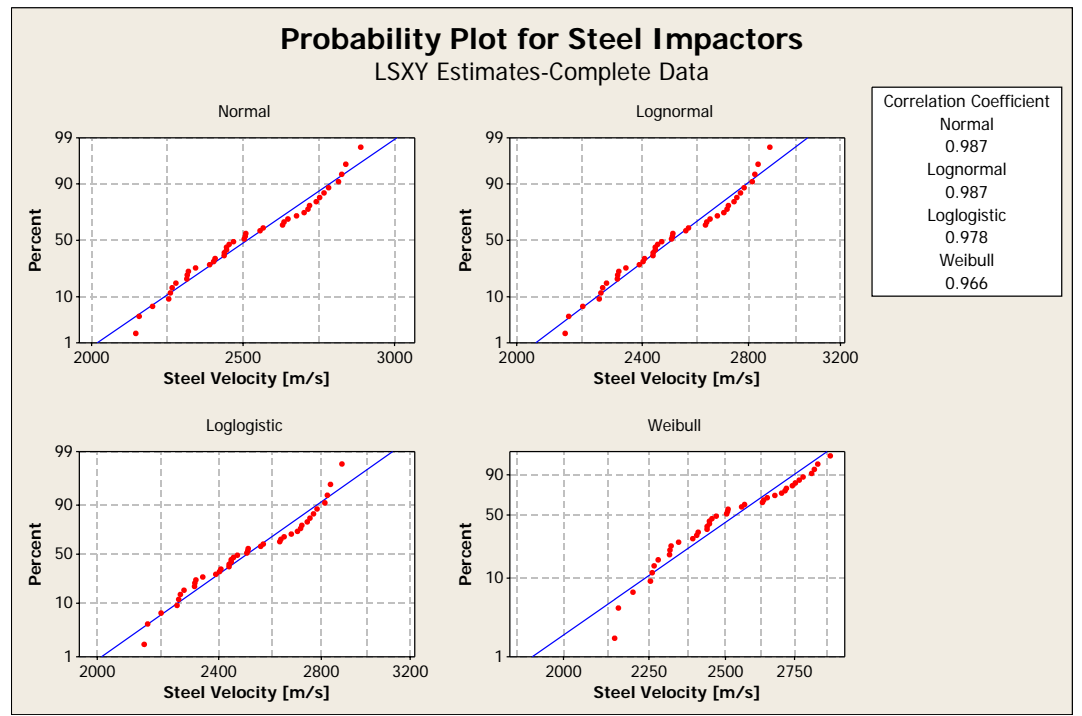

Figure A.4.7: Additional distributions fit to 440C steel spheres, $1.8 \mathrm{~mm}$ diameter, hypervelocimetry data 


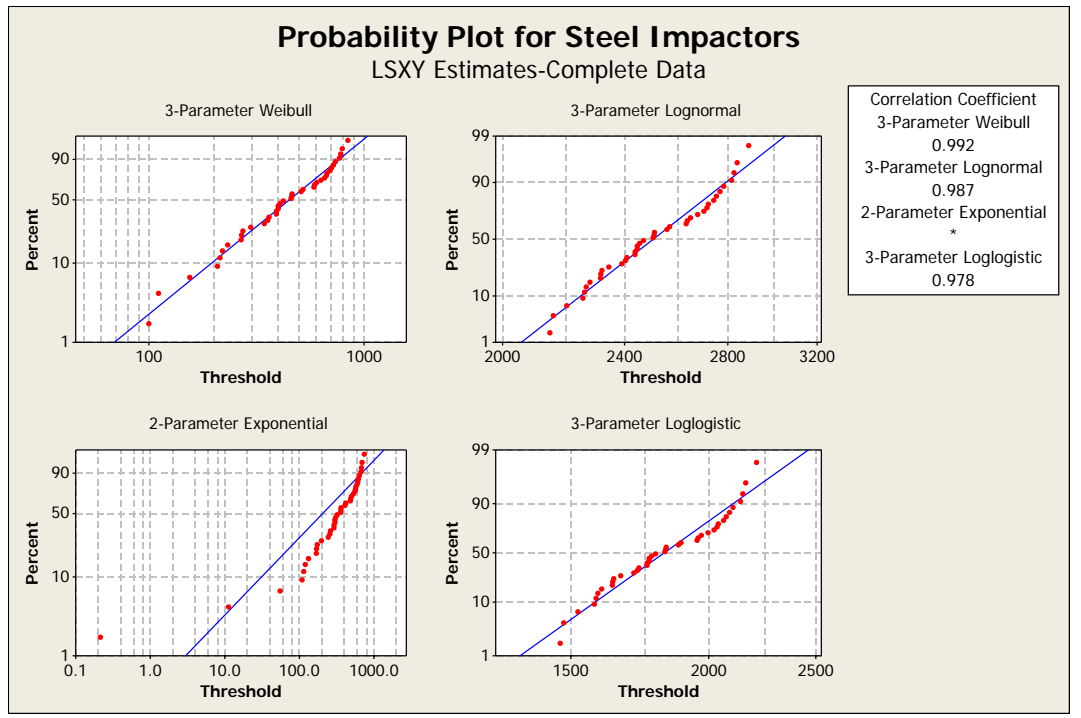

Figure A.4.8: Additional distributions fit to 440C steel spheres, $1.8 \mathrm{~mm}$ diameter, hypervelocimetry data

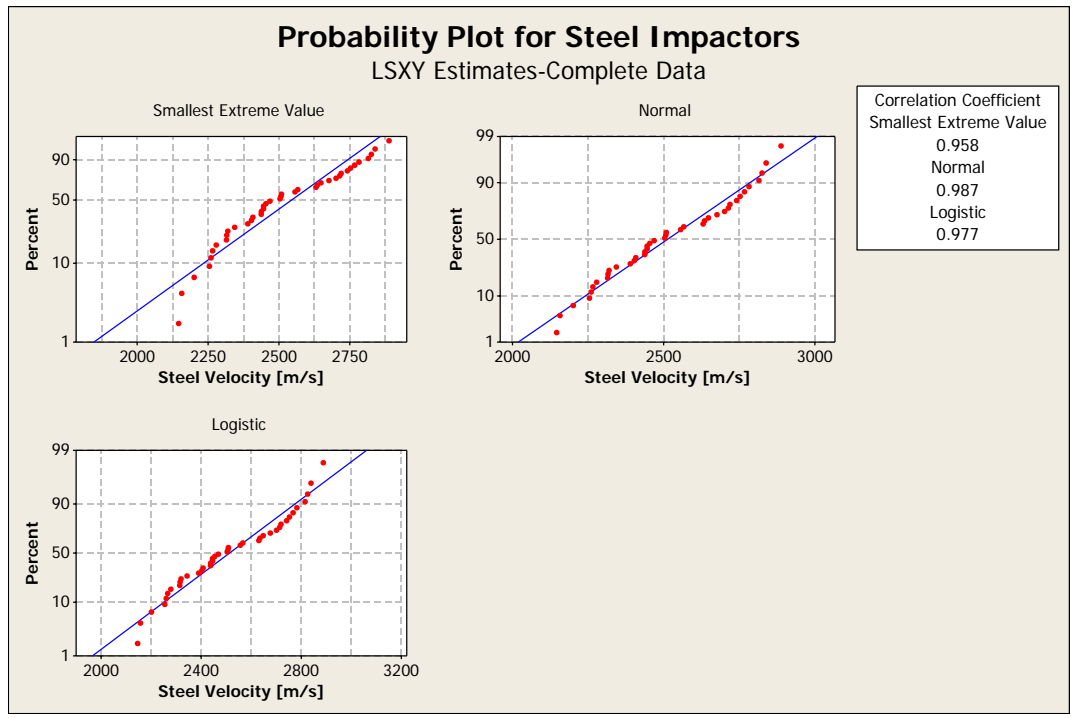

Figure A.4.9: Additional distributions fit to 440C steel spheres, $1.8 \mathrm{~mm}$ diameter, hypervelocimetry data 


\section{Appendix B}

\section{Additional Optical Investigation Details \& Extensions}

\section{B.1 Optical Distortion Removal Code}

The MATLAB script is as follows:

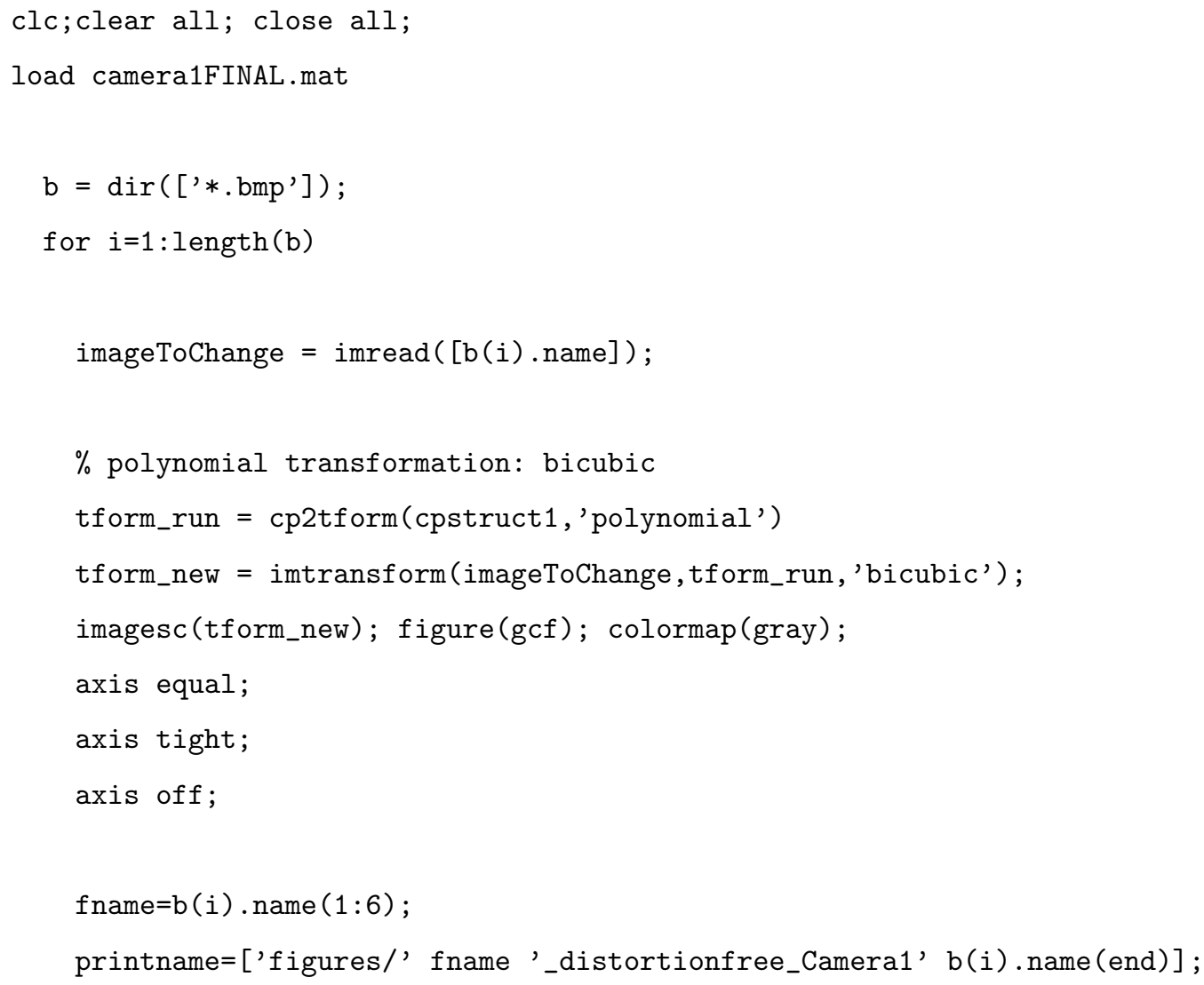




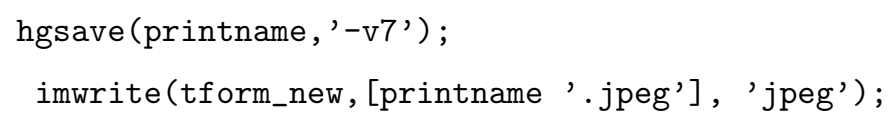

\section{B.2 Extensions of Presented Dynamic Optical Investigations}

There are countless related avenues of research regarding the dynamic optical investigations of hypervelocity impact damage that could be explored. A few of the most comparable to the work presented include examining damage accumulation from multiple impact strikes, extending optical diagnostics for comparison and possible additional quantitative information, as well as examining weak interfaces to begin to probe the relevance of classical dynamic fracture mechanics in mixed mode or mode-I, opening, and mode-II, shear, crack growth or crack loading scenarios.

The aforementioned additional research possibilities have been briefly probed, and qualitative results are shown in the following figures. 

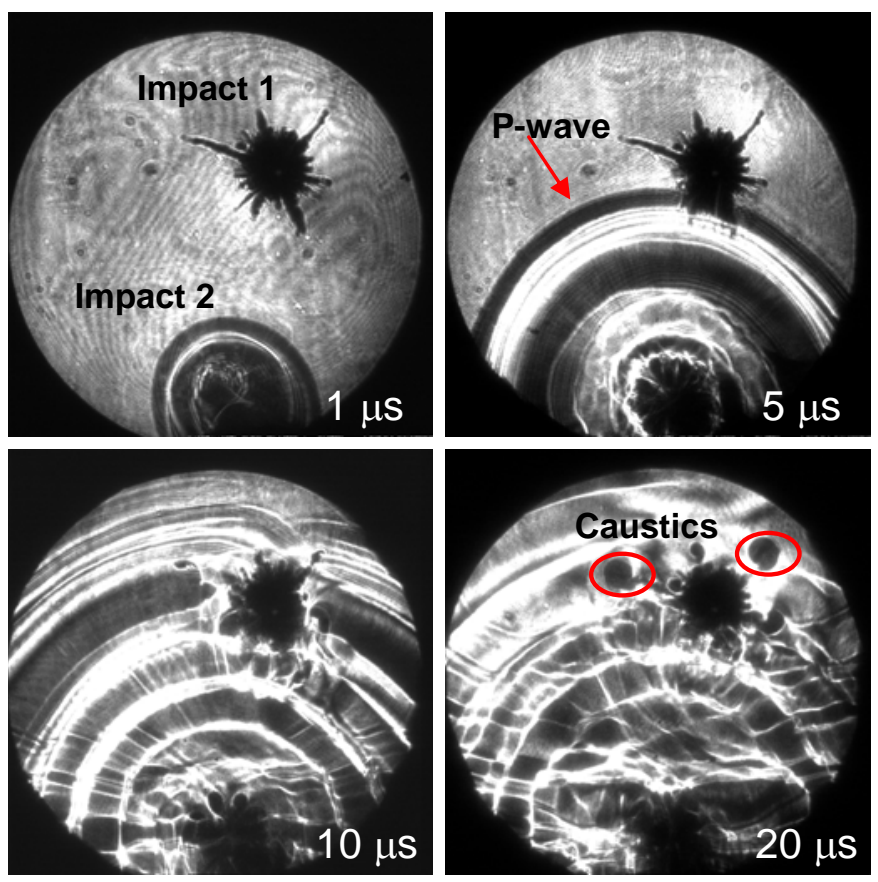

Figure B.2.1: A nylon 6-6 right cylindrical slug, length and diameter equal to $1.8 \mathrm{~mm}$ and a mass of $5 \mathrm{mg}$, impacts a Homalite 100 plate $6.5 \mathrm{~mm}$ thick that has existing hypervelocity impact damage. The second hypervelocity impact collided with the plate at $5 \mathrm{~km} / \mathrm{s}$ and the wave phenomena and interaction with the existing damage site is captured with high-speed photography and optical diagnostics. Imaging laser power is at 2 Watts with the beam expanded to a 100 mm diameter field of view, and exposure times are around 70 ns. After $5 \mu$ s the $\mathrm{P}$ wave from the second impact has reached the first impact site and soon after caustics from crack growth can be seen at both impact sites. Complex wave interaction and reflections from free surfaces is seen after $10 \mu \mathrm{s}$ 


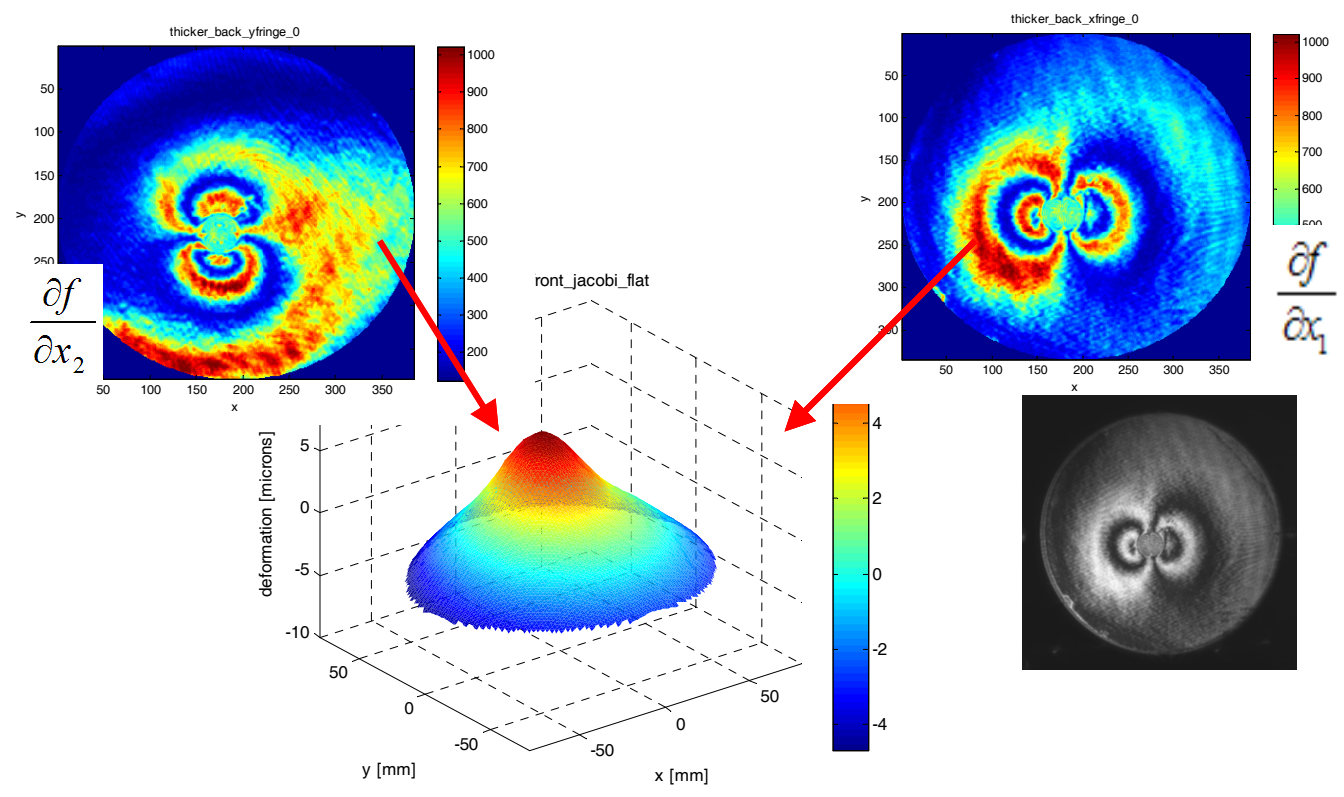

Figure B.2.2: Post-mortem Coherent Gradient Sensing, shearing interferometry technique, slope deformation mapping and 3-D topology results from hypervelocity impact of a nylon cylindrical slug, $1.8 \mathrm{~mm}$ in diameter and length, on a 304 steel target, $150 \mathrm{~mm}$ diameter and $10 \mathrm{~mm}$ thick, at $5.5 \mathrm{~km} / \mathrm{s}$ 


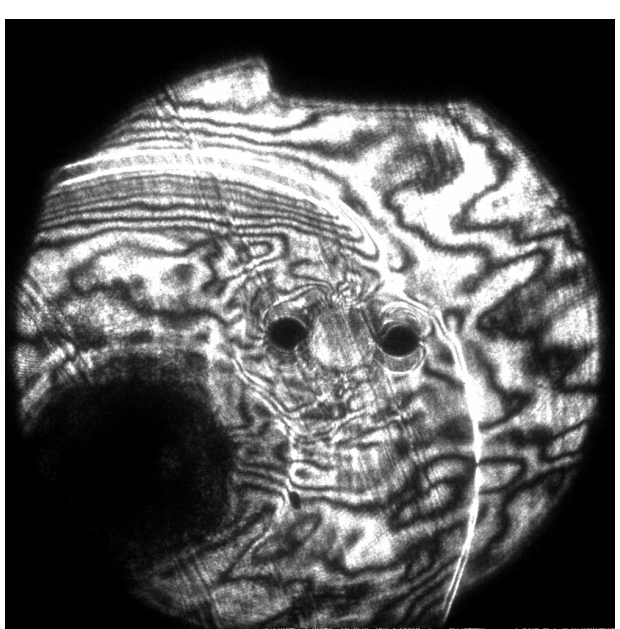

$\Delta=13 \mathrm{~cm}$

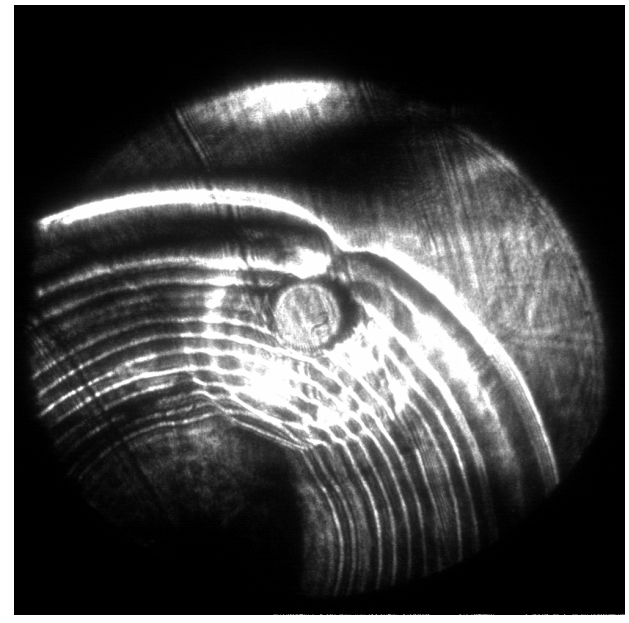

$\Delta=13 \mathrm{~mm}$

Figure B.2.3: In-situ Coherent Gradient Sensing (CGS), shearing interferometry technique, results in one-direction from hypervelocity impact of a nylon cylindrical slug on a brittle polymer plate at two different gratings separation. (Left) Mylar plate, $150 \mathrm{~mm}$ diameter and $1.6 \mathrm{~mm}$ with initial hole, pre-crack and 3.5 MPa tension pre-load, $30 \mu$ s after impact by nylon cylindrical slug at $4.56 \mathrm{~km} / \mathrm{s}$, and CGS gratings $13 \mathrm{~cm}$ apart. Vibrations in the impact and grating sensitivity in this case are such that the fidelity of the fringes at the crack tip are questionable. (Right) Homalite 100 plate $150 \mathrm{~mm}$ diameter and $1.6 \mathrm{~mm}$ thick, with initial hole, pre-crack and 1.65 MPa tension pre-load, $30 \mu$ s after impact by nylon cylindrical slug at $4.67 \mathrm{~km} / \mathrm{s}$, and CGS gratings $13 \mathrm{~mm}$ apart. In this case, the CGS gratings are placed where the fidelity of determining crack tip behavior has been washed out. Initial results indicate that this optical technique may not be a good method to receive quantitative information ahead of the moving crack in the configurations presented 


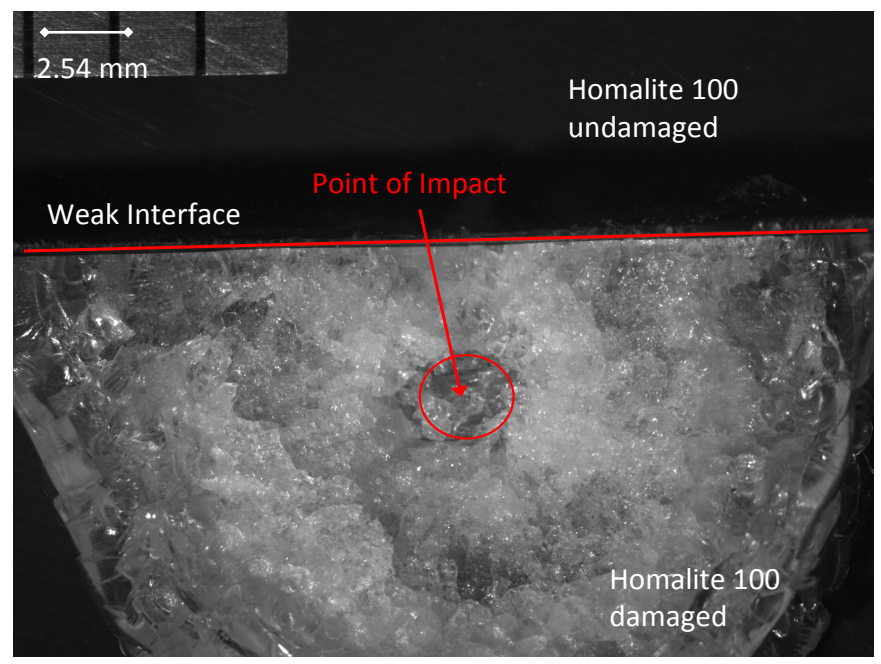

Figure B.2.4: Homalite 100, $10 \mathrm{~mm}$ thick, weakly interfaced with Homalite 100, also $10 \mathrm{~mm}$ thick, and impacted on the lower portion by a nylon cylindrical slug, $1.8 \mathrm{~mm}$ length and diameter, at $6.3 \mathrm{~km} / \mathrm{s}$. Notice the large damage zone is only observed in the lower piece of Homalite 100, and no damage can be seen in the upper piece across the interface 

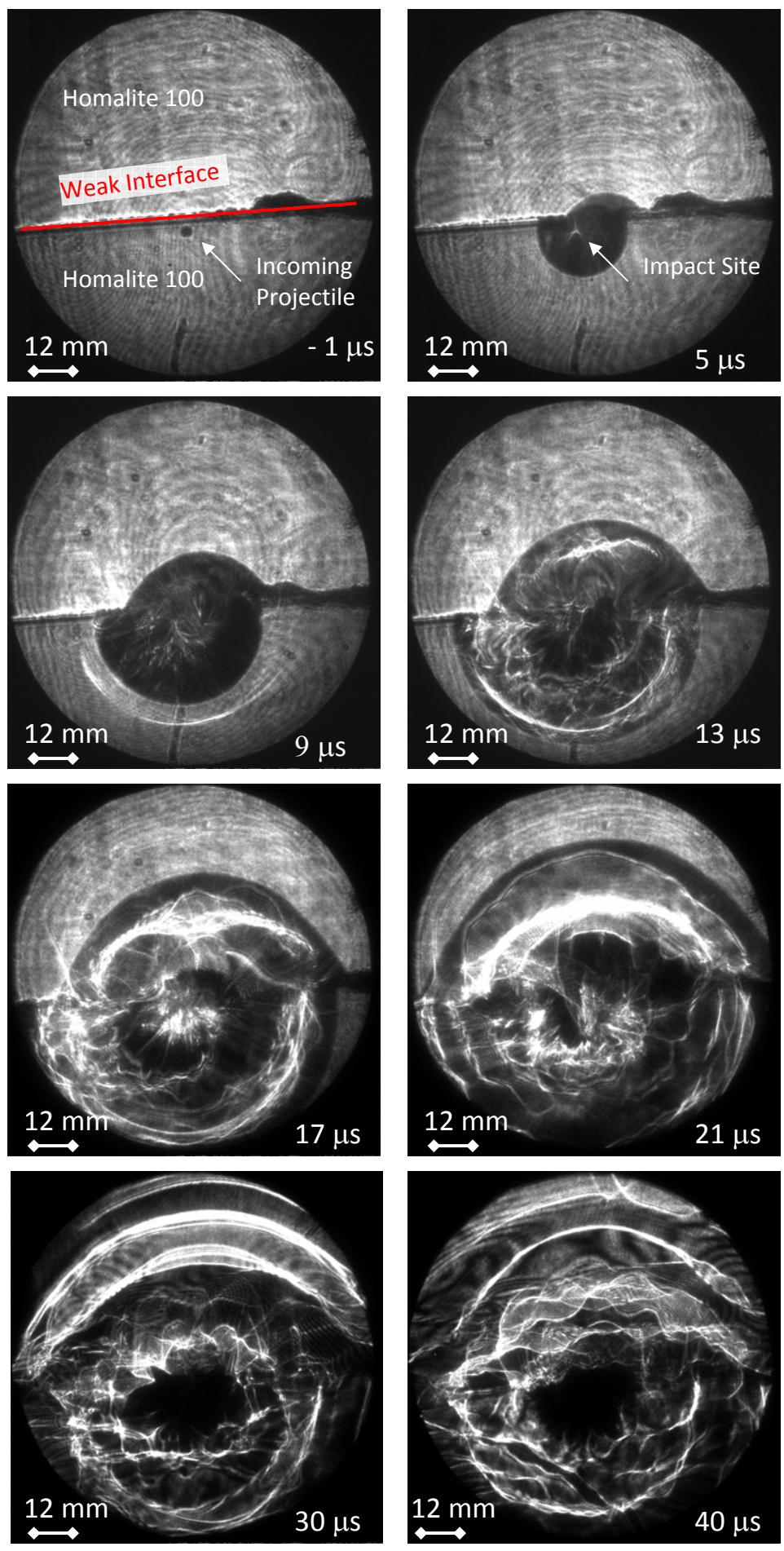

Figure B.2.5: Dynamic photoelasticity and caustic analysis of Homalite 100, $10 \mathrm{~mm}$ thick, weakly interfaced with Homalite 100, also $10 \mathrm{~mm}$ thick, and impacted on the lower section by a nylon cylindrical slug, $1.8 \mathrm{~mm}$ length and diameter, at $6.3 \mathrm{~km} / \mathrm{s}$. Waves propagate across interface, however, no damage can be seen on the upper section 


\section{Bibliography}

[1] NASA Orbital Debris Program Office webpage, 2010.

[2] (Anonymous). James Webb Space Telescope sunshield design achieves significant landmark. Associated Press, February 2010.

[3] J. Beinert and J.F. Kalthoff. Experimental determination of dynamic stress intensity factors by shadow patterns. In Experimental evaluation of stress concentration and intensity factors. Martinus Nijhoff, 1981.

[4] D.E. Carlucci and S.S. Jacobson. Ballistics: Theory and Design of Guns and Ammunition. CRC Press, 2008.

[5] C.J. Cornelison and E.T. Watts. Results of two-stage light-gas gun development efforts and hypervelocity impact tests of advanced thermal protection materials. Technical Report TM1998-112234, NASA, 1998.

[6] M. Cunn. International space station gets a bay window. sci-tech-today.com, February 2010.

[7] T. Kobayashi, J.W. Dally, and W.L. Fourney. Influence of specimen geometry on crack propagation and arrest behavior. In VIth International Conference of Expeirmental Stress Analysis, pages 18-22, 1978.

[8] G. Drolshagen. Impact effects from small size meteoroids and space debris. Advances in Space Research, 41:1123-1131, 2008.

[9] A.S. Kobayashi, A.F. Emery, and S. Mall. Dynamic finite element and dynamic photoelastic analyses of two fracturing Homalite-100 plates. Experimental Mechanics, 16:321-328, 1976.

[10] H.C. Koons, J.E. Mazur, R.S. Selesnick, et al. The impact of the space environment on space systems. Technical Report TR-99(1670)-1, The Aerospace Corporation, 1999.

[11] H.K. Tran, C.E. Johnson, et al. Phenolic impregnated carbon ablators (PICA) as thermal protection systems for discovery missions. Technical report, NASA Technical Memorandum 110440, 1997. 
[12] J. Mihaly, L. Lamberson, et al. A low-cost, small bore, light-gas gun facility. In Hypervelocity Impact Symposium, 2010.

[13] M. Grujicic, B. Pandurangan, et al. Hypervelocity impact resistance of reinforced carboncarbon/carbon-foam thermal protection systems. Applied Surface Science, 252:5035-5050, 2006.

[14] M.J. Starksa, D.L. Cooke, B.K. Dichtera, L.C. Chhabildas, et al. Seeking radio emissions from hypervelocity micrometeoroid impacts: Early experimental results from the ground. International Journal of Impact Engineering, 33:781-787, 2006.

[15] R. Destefanis, F. Schafer, et al. Selecting enhanced space debris shields for manned spacecraft. International Journal of Impact Engineering, 33:219-230, 2006.

[16] F. Figueroa. SolidWorks image. Created in Professor Veronica Eliasson's research group at the University of Southern California, 2010.

[17] L.B. Freund. Dynamic Fracture Mechanics. Cambridge University Press, 1990.

[18] E. Gdoutos. Fracture Mechanics Criteria and Applications. Springer, 1990.

[19] D.J. Grosch and J.P. Riegel. Development and optimization of a "micro" two-stage light-gas gun. In Hypervelocity Impact Proceedings Symposium, 1992.

[20] A. Hayter. Probability and statistics for engineers and scientists. Duxbury, 2007.

[21] C.R. Hicks and K.V. Turner, Jr. Fundamental Concepts in the Design of Experiments. Oxford University Press, Inc., 1999.

[22] S.A. Hill. Determination of an empirical model for the prediction of penetration hole diameter in thin plates from hypervelocity impact. International Journal of Impact Engineering, 30:303$321,2004$.

[23] G. Irwin. Analysis of stresses and strains near the end of a crack traversing plate. Journal of Applied Mechanics, 24:361-364, 1957.

[24] V. Isachenkov. Space crash debris could linger 10,000 years. Associated Press, February 2009.

[25] A.S. Kobayashi, editor. Handbook of Experimental Mechanics. Wiley, 1993.

[26] A.S. Kobayashi and J.W. Dally. Dynamic photoelastic determination of the a-K relation in 4344 alloy steel. In Crack Arrest Methodology and Applications, 1979.

[27] A.S. Koybayashi and S. Mall. Dynamic fracture toughness of Homalite-100. Experimental Mechanics, 18:11-18, 1978. 
[28] S. Krishnaswamy and A.J. Rosakis. On the extent of dominance of asymptotic elastodynamic crack-tip fields: Part I- an experimental study using bifocal caustics. Journal of Applied Mechanics, 58:87-94, 1990.

[29] L.R. Lamberson. Personal communications, 2008-2010.

[30] C.J. Maiden and A.R. McMillan. An investigation of the protection afforded a spacecraft by a thin shield. AIAA Journal, 2(11):1992-1998, 1964.

[31] T. Malik. Space shuttle tile system unique, future could bring alternatives. SPACE.com, March 2003.

[32] P.G. Mathews. Design of Experiments with MINITAB. ASQ Quality Press, 2004.

[33] C.J. Maiden, A.R. McMillian, and R.E. Sennett. Thin sheet impact. Technical report, NASA CR-295, 1965.

[34] H.J. Melosh. Impact Cratering: A Geological Process. Oxford University Press, Inc., 1989.

[35] W.A. Allen, G.E. Meloy, and J.W. Rogers. Hypervelocity precision impact instrument. The Review of Scientific Instruments, 31:726-731, 1960.

[36] D.C. Montgomery. Design and Analysis of Experiments. John Wiley \& Sons, Inc., 2001.

[37] S. Lai, E. Murad, and W. McNeil. Hazards on hypervelocity impacts on spacecraft. Journal of Spacecraft and Rockets, 39:106-114, 2002.

[38] C.P. Nysmith and B.P. Denardo. Experimental investigation of the momentum transfer associated with impact into thin aluminum targets. Technical report, NASA TN D-5492, 1969.

[39] Committee on International Space Station Meteoroid/Debris Risk Management. Protecting the Space Station from Meteoroids and Orbital Debris. National Academies Press, 1997.

[40] A.J. Piekutowski and K.L. Poormon. Impact of thin aluminum sheets with aluminum spheres at up to $9 \mathrm{~km} / \mathrm{s}$. International Journal of Impact Engineering, 35:1716-1722, 2008.

[41] D.F. Portree and J.P. Loftus, Jr. Orbital debris: A chronology. Technical Report TP-1999208856, NASA, 1999.

[42] R.F. Rolsten and H. Hunt. Phenomena resulting from hypervelocity impact. Journal of Spacecraft, 2:38-43, 1965.

[43] A.J. Rosakis. Analysis of the optical method of caustics for dynamic crack propagation. Engineering Fracture Mechanics, 13:331-347, 1980. 
[44] A.J. Rosakis and A.T. Zehnder. On the dynamic fracture of structural metals. Interational Journal of Fracture, 27:169, 1985.

[45] D.R. Sawle. Hypervelocity impact in thin sheets and semi-infinite targets at $15 \mathrm{~km} / \mathrm{s}$. AIAA Journal, 8:1240-1244, 1970.

[46] W.P. Schonberg and L.E. Compton. Application of NASA/JSC Whipple shield ballistic limit equations to dual-wall targets under hypervelocity impact. International Journal of Impact Engineering, 35:1792-1798, 2008.

[47] D.A. Shockey. Fracture of structural materials under dynamic loading. Technical Report AFOSR-TR-0402, Air Force Office of Scientific Research, March 1961.

[48] G.W. Snedecor and W.G. Cochran. Statistical Methods, Eighth Edition. Iowa State University Press, 1989.

[49] E.G. Stanberry and J.L. Foster. Monitoring the low earth orbit debris environment over an 11-year solar cycle. Advances in Space Research, 34:878-883, 2004.

[50] Office of Technology Assessment U.S. Congress. Orbiting debris: A space environmental problem-background paper. Technical Report OTA-BP-ISC-72, Washington, DC: U.S. Government Printing Office, September 1990.

[51] R.F. Rolsten, J.N. Wellnitz, and H.H. Hunt. An example of hole diameter in thin plates due to hypervelocity impact. Journal of Applied Physics, 35(3):556-559, 1964.

[52] H.A. Zook. Spacecraft Measurements of Cosmic Dust Flux, chapter 5, pages 75-92. Plenum Publishers, 2001. 